\title{
Unsteady non-Newtonian fluid flow with heat transfer and Tresca's friction boundary conditions
}

\section{Laetitia Paoli ${ }^{1 *}$ (i)}

\section{"Correspondence:}

laetitia.paoli@univ-st-etienne.fr

1 Universite Jean Monnet,

Saint-Etienne, France

\begin{abstract}
We consider an unsteady non-isothermal flow problem for a general class of non-Newtonian fluids. More precisely the stress tensor follows a power law of parameter $p$, namely $\sigma=2 \mu(\theta, v,\|D(v)\|)\|D(v)\|^{p-2} D(v)-\pi$ Id where $\theta$ is the temperature, $\pi$ is the pressure, $v$ is the velocity, and $D(v)$ is the strain rate tensor of the fluid. The problem is then described by a non-stationary $p$-Laplacian Stokes system coupled to an $L^{1}$-parabolic equation describing thermal effects in the fluid. We also assume that the velocity field satisfies non-standard threshold slip-adhesion boundary conditions reminiscent of Tresca's friction law for solids. First, we consider an approximate problem $\left(P_{\delta}\right)$, where the $L^{1}$ coupling term in the heat equation is replaced by a bounded one depending on a small parameter $0<\delta \ll 1$, and we establish the existence of a solution to $\left(P_{\delta}\right)$ by using a fixed point technique. Then we prove the convergence of the approximate solutions to a solution to our original fluid flow/heat transfer problem as $\delta$ tends to zero.
\end{abstract}

MSC: Primary 76A05; 35Q35; secondary 35Q79; 35K87; 76M30

Keywords: Unsteady non-Newtonian fluid flow; Shear thickening and shear thinning fluids; Heat transfer; Tresca's friction boundary conditions; Non-linear parabolic variational inequality; Fixed point technique

\section{Springer}

\section{Introduction}

Motivated by lubrication or injection/extrusion industrial processes, we consider in this paper an unsteady incompressible non-isothermal flow problem with non-linear boundary conditions of friction type for a general class of non-Newtonian fluids. More precisely, we assume that the stress tensor is given by

$$
\sigma=2 \mu(\theta, v,\|D(v)\|)\|D(v)\|^{p-2} D(v)-\pi \operatorname{Id}_{\mathbb{R}^{3}},
$$

where $\mu$ is a given mapping, $\theta$ is the temperature, $\pi$ is the pressure, $v$ is the velocity, $D(v)$ is the strain rate tensor, and $p \in(1,+\infty)$ is a real parameter.

(c) The Author(s) 2022. This article is licensed under a Creative Commons Attribution 4.0 International License, which permits use, sharing, adaptation, distribution and reproduction in any medium or format, as long as you give appropriate credit to the original author(s) and the source, provide a link to the Creative Commons licence, and indicate if changes were made. The images or other third party material in this article are included in the article's Creative Commons licence, unless indicated otherwise in a credit line to the material. If material is not included in the article's Creative Commons licence and your intended use is not permitted by statutory regulation or exceeds the permitted use, you will need to obtain permission directly from the copyright holder. To view a copy of this licence, visit http://creativecommons.org/licenses/by/4.0/. 
When $p>2$, this non-linear power-law models the behavior of dilatant (or shear thickening) fluids like colloidal fluids, while the case $p \in(1,2)$ gives a description of pseudo-plastic (or shear thinning) fluids like molten polymers [1, 3, 25, 30, 33, 37].

When $p=2$, the relationship between the stress tensor, the strain rate tensor and the pressure is still non-linear since the viscosity mapping $\mu$ depends on $\theta, v$, and $|D(v)|$, and we obtain a constitutive law that allows considering non-Newtonian fluids like oils [22].

Let us mention that (1.1) corresponds to a quasi-linear version of the Newton constitutive law and is also called the generalized Newtonian fluid model.

Several experimental studies have shown that such complex fluids exhibit a nonstandard behavior at the boundary with threshold slip-adhesion phenomena reminiscent of Tresca's friction law for solids [4, 13, 21, 26, 27, 35]. The first existence results for this kind of boundary conditions have been obtained by H. Fujita in [14-19, 31, 32] for stationary Newtonian Stokes flows and developed later on for steady and unsteady Newtonian fluid flows [7, 8, 23, 24, 36].

The case of stationary non-Newtonian fluids satisfying the general power law (1.1) is considered in [9], and thermal effects lead to a coupled fluid flow/heat transfer problem.

This paper aims to extend this result to the non-stationary case. More precisely, we consider the fluid flow domain

$$
\Omega=\left\{\left(x^{\prime}, x_{3}\right) \in \mathbb{R}^{2} \times \mathbb{R}: x^{\prime} \in \omega, 0<x_{3}<h\left(x^{\prime}\right)\right\}
$$

where $\omega$ is a non-empty bounded domain of $\mathbb{R}^{2}$ with a Lipschitz continuous boundary, and $h$ is a Lipschitz continuous function bounded from above and below by some positive real numbers.

The conservation of mass and momentum and the energy conservation law yield the following $p$-Laplacian Stokes system:

$$
\left\{\begin{array}{l}
\frac{\partial v}{\partial t}-2 \operatorname{div}\left(\mu(\theta, v,\|D(v)\|)\|D(v)\|^{p-2} D(v)\right)+\nabla \pi=f \quad \text { in }(0, T) \times \Omega \\
\operatorname{div}(v)=0 \quad \text { in }(0, T) \times \Omega
\end{array}\right.
$$

where $(0, T)$ is a non-trivial time interval, and $f$ describes the external forces coupled to the following heat equation:

$$
c \frac{\partial \theta}{\partial t}-\operatorname{div}(K \nabla \theta)=2 \mu(\theta, v,\|D(v)\|)\|D(v)\|^{p}+r(\theta) \quad \text { in }(0, T) \times \Omega,
$$

where $c$ is the heat capacity, $K$ is the thermal conductivity tensor, and $r$ is a real function.

Let us observe that we take into account only two kinds of coupling effects in this description of the fluid flow/heat transfer problem. More precisely, in (1.2), the viscosity mapping $\mu$ depends on the temperature, and in (1.3), the right-hand side contains the heat source term $2 \mu(\theta, v,\|D(v)\|)\|D(v)\|^{p}$, which describes the heat generation due to inner friction. Indeed, in this first attempt for an existence result generalizing [9] to the non-stationary case, we focus in this paper on the difficulty due to the $L^{1}$ right-hand side in (1.3), which will be dealt with through the truncation technique introduced by L. Boccardo and T. Gallouët in [6].

Hence, we choose to neglect convective effects both in the momentum equation and in the heat equation. A more complete description, including the convective terms, could 
be handled with the same proof strategy (with more technicalities) under some restrictive conditions on the value of the parameter $p$ and some compatibility conditions between the regularity properties of the fluid velocity and temperature fields. Similarly, we choose to neglect the semilinear temperature-dependent term modeling the buoyancy force in the momentum equation.

We decompose the boundary of $\Omega$ as $\partial \Omega=\Gamma_{0} \cup \Gamma_{L} \cup \Gamma_{1}$ with

$$
\Gamma_{0}=\left\{\left(x^{\prime}, x_{3}\right) \in \bar{\Omega}: x_{3}=0\right\}, \quad \Gamma_{1}=\left\{\left(x^{\prime}, x_{3}\right) \in \bar{\Omega}: x_{3}=h\left(x^{\prime}\right)\right\},
$$

and $\Gamma_{L}$ is the lateral part of $\partial \Omega$. We introduce a function $g: \partial \Omega \rightarrow \mathbb{R}^{3}$ such that

$$
\int_{\Gamma_{L}} g \cdot n d Y=0, \quad g=0 \quad \text { on } \Gamma_{1}, \quad g \cdot n=0 \quad \text { on } \Gamma_{0}, \quad g \neq 0 \quad \text { on } \Gamma_{L},
$$

where $n=\left(n_{1}, n_{2}, n_{3}\right)$ is the unit outward normal vector to $\partial \Omega$, and $g \cdot n$ denotes the Euclidean inner product of the vectors $g$ and $n$ in $\mathbb{R}^{3}$. We define by $v_{n}=v \cdot n$ and $v_{\tau}=v-v_{n} n$ the normal and the tangential velocities on $\partial \Omega$. The normal and tangential components of the stress vector on $\partial \Omega$ are given by $\sigma_{n}$ and $\sigma_{\tau}$ with

$$
\sigma_{n}=\sum_{i, j=1}^{3} \sigma_{i j} n_{j} n_{i}, \quad \sigma_{\tau}=\left(\sum_{j=1}^{3} \sigma_{i j} n_{j}-\sigma_{n} n_{i}\right)_{1 \leq i \leq 3} .
$$

As usual in lubrication or extrusion/injection problems, the upper part of the boundary is a fixed wall, while the lower part is a moving device. Hence, we assume that the fluid is subjected to the non-homogeneous Dirichlet boundary conditions on $\Gamma_{1} \cup \Gamma_{L}$ and to non-linear slip boundary conditions of friction type on $\Gamma_{0}$, i.e.,

$$
v=0 \quad \text { on }(0, T) \times \Gamma_{1}, \quad v=g \xi \quad \text { on }(0, T) \times \Gamma_{L},
$$

where $\xi$ is a function depending only on the time variable such that

$$
\xi(0)=1
$$

and

$$
\begin{aligned}
& v_{n}=0 \quad \text { on }(0, T) \times \Gamma_{0} \quad \text { (slip condition), } \\
& \left.\begin{array}{lll}
\left\|\sigma_{\tau}\right\|=k \quad \Rightarrow \quad \exists \lambda \geq 0 & v_{\tau}=s-\lambda \sigma_{\tau}, \\
\left\|\sigma_{\tau}\right\|<k \quad \Rightarrow \quad v_{\tau}=s &
\end{array}\right\} \quad \text { on }(0, T) \times \Gamma_{0} \quad \text { (Tresca's law), }
\end{aligned}
$$

where $s$ is the sliding velocity of the lower part of the boundary, and $k$ is the positive friction threshold.

Moreover, we assume the mixed Dirichlet-Neumann boundary conditions on $\Gamma_{1} \cup \Gamma_{L}$ and $\Gamma_{0}$ for the temperature, i.e.,

$$
\theta=0 \quad \text { on }(0, T) \times\left(\Gamma_{1} \cup \Gamma_{L}\right), \quad(K \nabla \theta) \cdot n=\theta^{b} \quad \text { on }(0, T) \times \Gamma_{0},
$$

where $\theta^{b}$ is a given heat flux on $\Gamma_{0}$. 
The paper is organized as follows: In Sect. 2, we introduce the functional framework and derive the mathematical formulation of the problem as a non-linear parabolic variational inequality for the velocity and pressure fields coupled to a non-linear parabolic equation for the temperature. By observing that the right-hand side of the heat equation belongs to $L^{1}\left(0, T ; L^{1}(\Omega)\right)$, we introduce in Sect. 3 an approximate problem $\left(P_{\delta}\right)$, where the $L^{1}$ coupling term is replaced by a bounded one depending on a small parameter $0<\delta \ll 1$, and we establish the existence of a solution to $\left(P_{\delta}\right)$ by using a fixed point technique. Finally, in Sect. 4 , we prove that the approximate solutions $\left(v_{\delta}, \pi_{\delta}, \theta_{\delta}\right)$ converge to a solution to our original fluid flow/heat transfer problem as $\delta$ tends to zero.

\section{Mathematical formulation of the problem}

Throughout the paper, we will denote by $\mathbf{X}$ the functional space $X^{3}$.

In order to describe the fluid flow problem, we introduce the following subspaces of $\mathbf{W}^{1, p}(\Omega)$ :

$$
\begin{aligned}
& V_{\Gamma_{1}}^{p}=\left\{\varphi \in \mathbf{W}^{1, p}(\Omega) ; \varphi=0 \text { on } \Gamma_{1}\right\}, \\
& V_{0}^{p}=\left\{\varphi \in \mathbf{W}^{1, p}(\Omega) ; \varphi=0 \text { on } \Gamma_{1} \cup \Gamma_{L} \text { and } \varphi \cdot n=0 \text { on } \Gamma_{0}\right\}
\end{aligned}
$$

and

$$
V_{0 . \text { div }}^{p}=\left\{\varphi \in V_{0}^{p} ; \operatorname{div}(\varphi)=0 \text { in } \Omega\right\}
$$

for all $p>1$ endowed with the norm

$$
\|v\|_{1 \cdot p}=\left(\int_{\Omega}\|\nabla v\|^{p} d x\right)^{1 / p} .
$$

By using the convexity of the mapping $z \mapsto z^{p}$ on $\mathbb{R}_{*}^{+}$, we obtain

$$
\left(\int_{\Omega}\|D(u)\|^{p} d x\right)^{1 / p}=\|D(u)\|_{\left(L^{p}(\Omega)\right)^{3 \times 3}} \leq\|u\|_{1 \cdot p} \quad \forall u \in \mathbf{W}^{1, p}(\Omega),
$$

where $D(u)=\left(d_{i j}(u)\right)_{1 \leq i, j \leq 3}=\left(\frac{1}{2}\left(\frac{\partial u_{i}}{\partial x_{j}}+\frac{\partial u_{j}}{\partial x_{i}}\right)\right)_{1 \leq i, j \leq 3}$, and with Korn's inequality [38], we have

$$
\left(\int_{\Omega}\|D(u)\|^{p} d x\right)^{1 / p}=\|D(u)\|_{\left(L^{p}(\Omega)\right)^{3 \times 3}} \geq C_{\mathrm{Korn}, p}\|u\|_{1 \cdot p} \quad \forall u \in V_{\Gamma_{1}}^{p}
$$

where $C_{\mathrm{Korn}, p}>0$. Moreover, let $\mathcal{Y}=\left\{\psi \in \mathbf{L}^{2}(\Omega) ; \operatorname{div}(\psi) \in L^{2}(\Omega)\right\}$ endowed with its canonical norm

$$
\|\psi\|_{\mathcal{Y}}=\left(\|\psi\|_{\mathbf{L}^{2}(\Omega)}^{2}+\|\operatorname{div}(\psi)\|_{L^{2}(\Omega)}^{2}\right)^{1 / 2} \quad \forall \psi \in \mathcal{Y},
$$

and let $H$ be its subspace given by $H=\left\{\psi \in \mathbf{L}^{2}(\Omega) ; \operatorname{div}(\psi)=0\right.$ in $\Omega, \psi \cdot n=0$ on $\left.\partial \Omega\right\}$. Owing to that $\mathbf{W}^{1, p}(\Omega)$ is continuously embedded into $\mathbf{L}^{2}(\Omega)$ if and only if $p \geq \frac{6}{5}$ in a $3 \mathrm{D}$ setting and that the space $\mathcal{V}=\left\{\Phi \in(\mathcal{D}(\Omega))^{3} ; \operatorname{div}(\Phi)=0\right.$ in $\left.\Omega\right\}$ is dense in $H$ (see Chap. 1, Theorem 2.8 in [20]), we obtain that the embedding of $V_{0 \text {.div }}^{p}$ into $H$ is continuous and 
dense if and only if $p \geq \frac{6}{5}$, and $\left(V_{0 . \operatorname{div}}^{p}, H,\left(V_{0 . \operatorname{div}}^{p}\right)^{\prime}\right)$ is a Gelfand triplet. Let us also recall that the trace operator is compact from $\mathbf{W}^{1, p}(\Omega)$ into $\mathbf{L}^{p}(\partial \Omega)$ for all $p>1$ (see Theorem 1.23 in [29]), which will allow us to deal with the boundary friction term (see (2.13) and the definition of the mapping $J$ ).

Similarly, we let

$$
W_{\Gamma_{1} \cup \Gamma_{L}}^{1, q}(\Omega)=\left\{\varphi \in W^{1, q}(\Omega): \varphi=0 \text { on } \Gamma_{1} \cup \Gamma_{L}\right\}
$$

for all $q>1$ endowed with the norm

$$
\|\varphi\|_{W_{\Gamma_{1} \cup \Gamma_{L}}^{1, q}(\Omega)}=\left(\int_{\Omega}\|\nabla \varphi\|^{q} d x\right)^{1 / q} .
$$

Let us now introduce the assumptions on the data.

The heat capacity and the thermal conductivity tensor satisfy

$$
\begin{aligned}
& c \in W^{1, q^{\prime}}(\Omega), \quad K \in\left(L^{\infty}(\Omega)\right)^{3 \times 3} \\
& \text { there exists }\left(c_{0}, c_{1}\right) \in \mathbb{R}^{2} \text { such that } \\
& 0<c_{0} \leq c(x) \leq c_{1} \quad \text { for a.e. } x \in \Omega,
\end{aligned}
$$

where $q^{\prime}=\frac{q}{q-1}$ is the conjugate number of $q$, and

there exists $k_{0}>0$ such that $\sum_{i, j=1}^{3} K_{i j}(x) \gamma_{i} \gamma_{j} \geq k_{0} \sum_{i=1}^{3}\left|\gamma_{i}\right|^{2}$

for all $\gamma=\left(\gamma_{i}\right)_{1 \leq i \leq 3} \in \mathbb{R}^{3}$ for a.e. $x \in \Omega$.

We also assume that

the mapping $r: \mathbb{R} \rightarrow \mathbb{R}$ is continuous,

and

there exists $r_{1} \in \mathbb{R}$ such that $|r(z)| \leq r_{1} \quad$ for all $z \in \mathbb{R}$.

The viscosity mapping $\mu: \mathbb{R} \times \mathbb{R}^{3} \times \mathbb{R}_{+} \rightarrow \mathbb{R}$ satisfies

$$
(o, e, d) \mapsto \mu(o, e, d) \text { is continuous on } \mathbb{R} \times \mathbb{R}^{3} \times \mathbb{R}_{+} \text {, }
$$

$d \mapsto \mu(\cdot, \cdot, d)$ is monotone increasing on $\mathbb{R}_{+}$,

there exists $\left(\mu_{0}, \mu_{1}\right) \in \mathbb{R}^{2}$ such that

$0<\mu_{0} \leq \mu(o, e, d) \leq \mu_{1} \quad$ for all $(o, e, d) \in \mathbb{R} \times \mathbb{R}^{3} \times \mathbb{R}_{+}$,

and we define $\mathcal{F}: \mathbb{R} \times \mathbb{R}^{3} \times \mathbb{R}^{3 \times 3} \rightarrow \mathbb{R}^{3 \times 3}$ by

$$
\left\{\begin{array}{l}
\mathcal{F}\left(\lambda_{0}, \lambda_{1}, \lambda_{2}\right)=2 \mu\left(\lambda_{0}, \lambda_{1},\left\|\lambda_{2}\right\|\right)\left\|\lambda_{2}\right\|^{p-2} \lambda_{2} \quad \text { if } \lambda_{2} \neq 0_{\mathbb{R}^{3 \times 3}}, \\
\mathcal{F}\left(\lambda_{0}, \lambda_{1}, \lambda_{2}\right)=0_{\mathbb{R}^{3 \times 3}} \quad \text { otherwise. }
\end{array}\right.
$$


With (2.10), we obtain immediately

$$
\left|\mathcal{F}\left(\lambda_{0}, \lambda_{1}, \lambda_{2}\right)\right| \leq 2 \mu_{1}\left\|\lambda_{2}\right\|^{p-1} \quad \forall\left(\lambda_{0}, \lambda_{1}, \lambda_{2}\right) \in \mathbb{R} \times \mathbb{R}^{3} \times \mathbb{R}^{3 \times 3} .
$$

Let $\tilde{p}>1$ and $p>1$ such that $\tilde{p}-p+1>0$. Then for any $\theta \in L^{\tilde{q}}\left(0, T ; L^{q}(\Omega)\right)$ with $\tilde{q} \geq 1$ and $q \geq 1$, and for any $u \in L^{\tilde{p}}\left(0, T ; \mathbf{W}^{1, p}(\Omega)\right)$, we have

$$
\mathcal{F}(\theta, u, D(u)) \in L^{\frac{\tilde{p}}{p-1}}\left(0, T ;\left(L^{p^{\prime}}(\Omega)\right)^{3 \times 3}\right),
$$

where $p^{\prime}=\frac{p}{p-1}$ is the conjugate number of $p$. Hence,

$$
\mathcal{F}(\theta, u, D(u)): D(\bar{\varphi}) \in L^{1}\left(0, T ; L^{1}(\Omega)\right)
$$

for all $\bar{\varphi} \in L^{\frac{\tilde{p}}{p-p+1}}\left(0, T ; \mathbf{W}^{1, p}(\Omega)\right)$, and the right-hand side of the heat equation is well defined in $L^{1}\left(0, T ; L^{1}(\Omega)\right)$ if and only if $\tilde{p} \geq \frac{\tilde{p}}{\tilde{p}-p+1}$, i.e., $\tilde{p} \geq p$. Then we may expect $\theta \in$ $L^{q}\left(0, T ; W_{\Gamma_{1} \cup \Gamma_{L}}^{1}(\Omega)\right)$ with $1<q<\frac{5}{4}$ (see $\left.[6]\right)$.

Let us introduce the operator $\mathcal{A}: L^{\tilde{p}}\left(0, T ; V_{\Gamma_{1}}^{p}\right) \rightarrow\left(L^{\tilde{p}}\left(0, T ; V_{\Gamma_{1}}^{p}\right)\right)^{\prime}$ defined by

$$
[\mathcal{A}(u), \bar{\varphi}]=\int_{0}^{T} \int_{\Omega} \mathcal{F}(\theta, u, D(u)): D(\bar{\varphi}) d x d t \quad \forall(u, \bar{\varphi}) \in\left(L^{\tilde{p}}\left(0, T ; V_{\Gamma_{1}}^{p}\right)\right)^{2},
$$

where $[\cdot, \cdot]$ denotes the duality product between the space $L^{\tilde{p}}\left(0, T ; V_{\Gamma_{1}}^{p}\right)$ and its dual $\left(L^{\tilde{p}}\left(0, T ; V_{\Gamma_{1}}^{p}\right)\right)^{\prime}$. With $(2.10)$, we have

$$
\begin{aligned}
|[\mathcal{A}(u), \bar{\varphi}]| & \leq 2 \mu_{1}\|D(u)\|_{L^{\tilde{p}}\left(0, T ; L^{p}(\Omega)\right)}^{p-1}\|D(\bar{\varphi})\|_{L^{\frac{\tilde{p}}{\tilde{p} p+1}}\left(0, T ; L^{p}(\Omega)\right)} \\
& \leq 2 \mu_{1} T^{\frac{\tilde{p}-p}{\tilde{p}}}\|u\|_{L^{\tilde{p}}\left(0, T ; V_{\Gamma_{1}}^{p}\right)}^{p-1}\|\bar{\varphi}\|_{L^{\tilde{p}}\left(0, T ; V_{\Gamma_{1}}^{p}\right)}
\end{aligned}
$$

and

$$
[\mathcal{A}(u), u-\bar{\varphi}] \geq 2\left(C_{\mathrm{Korn}, p}\right)^{p} \mu_{0}\|u\|_{L^{p}\left(0, T ; V_{\Gamma_{1}}^{p}\right)}^{p}-2 \mu_{1}\|u\|_{L_{\tilde{p}}\left(0, T ; V_{\Gamma_{1}}^{p}\right)}^{p-1}\|\bar{\varphi}\| \underset{L^{\frac{\tilde{p}}{\tilde{p}-p+1}}\left(0, T ; V_{\Gamma_{1}}^{p}\right)}{ }
$$

for any $(u, \bar{\varphi}) \in\left(L^{\tilde{p}}\left(0, T ; V_{\Gamma_{1}}^{p}\right)\right)^{2}$. It follows that $\mathcal{A}$ is a bounded operator and is coercive when $\tilde{p}=p$.

Hence, from now on, we will assume that $\tilde{p}=p \geq \frac{6}{5}$ and $q \in\left(1, \frac{5}{4}\right)$.

Let $v^{0} \in \mathbf{W}^{1, p}(\Omega)$ be a given initial velocity such that

$$
\begin{aligned}
& \operatorname{div}\left(v^{0}\right)=0 \quad \text { in } \Omega, \quad v^{0}=0 \quad \text { on } \Gamma_{1}, \quad v^{0}=g \quad \text { on } \Gamma_{L}, \\
& v^{0} \cdot n=0 \quad \text { on } \Gamma_{0} .
\end{aligned}
$$

In order to deal with homogeneous boundary conditions on $(0, T) \times\left(\Gamma_{1} \cup \Gamma_{L}\right)$, we set $\bar{v}=v-v^{0} \xi$. We obtain the following weak formulation of the problem:

Problem (P) Let $c, K, r$, and $\mu$ satisfy (2.3)-(2.10). Let $f \in L^{p^{\prime}}\left(0, T ; \mathbf{L}^{2}(\Omega)\right), k \in L^{p^{\prime}}(0, T$; $\left.L_{+}^{p^{\prime}}\left(\Gamma_{0}\right)\right), s \in L^{p}\left(0, T ; \mathbf{L}^{p}\left(\Gamma_{0}\right)\right), \xi \in W^{1, p^{\prime}}(0, T)$ satisfying $(1.6), \theta^{b} \in L^{1}((0, T) \times \omega), \theta^{0} \in L^{1}(\Omega)$ and $v^{0} \in \mathbf{W}^{1, p}(\Omega)$ satisfying (2.12). 
Find $\theta \in L^{q}\left(0, T ; W_{\Gamma_{1} \cup \Gamma_{L}}^{1, q}(\Omega)\right), \bar{v} \in C\left([0, T] ; \mathbf{L}^{2}(\Omega)\right) \cap L^{p}\left(0, T ; V_{0 . \text { div }}^{p}\right)$ with $\frac{\partial \bar{v}}{\partial t} \in L^{p^{\prime}}(0, T$; $\left.\left(V_{0 \text {.div }}^{p}\right)^{\prime}\right)$ and $\pi \in H^{-1}\left(0, T ; L_{0}^{p^{\prime}}(\Omega)\right)$ satisfying the following parabolic variational coupled problem:

$$
\begin{aligned}
\left\langle\frac{\partial}{\partial t}(\bar{v}, \tilde{\vartheta})_{\mathbf{L}^{2}(\Omega)}, \zeta\right\rangle_{\mathcal{D}^{\prime}(0, T), \mathcal{D}(0, T)} & \\
& +\int_{0}^{T} \int_{\Omega} \mathcal{F}\left(\theta, \bar{v}+v^{0} \xi, D\left(\bar{v}+v^{0} \xi\right)\right): D(\tilde{\vartheta}) \zeta d x d t \\
& -\left\langle\int_{\Omega} \pi \operatorname{div}(\tilde{\vartheta}) d x, \zeta\right\rangle_{\mathcal{D}^{\prime}(0, T), \mathcal{D}(0, T)}+J(\bar{v}+\tilde{\vartheta} \zeta)-J(\bar{v}) \\
\geq & \int_{0}^{T}\left(f+v^{0} \frac{\partial \xi}{\partial t}, \tilde{\vartheta}\right)_{\mathbf{L}^{2}(\Omega)} \zeta d t \quad \forall \tilde{\vartheta} \in V_{0}^{p}, \forall \zeta \in \mathcal{D}(0, T)
\end{aligned}
$$

with the initial condition

$$
\bar{v}(0, \cdot)=v^{0}-v^{0} \xi(0)=0 \quad \text { in } \Omega
$$

and

$$
\begin{aligned}
& -\int_{0}^{T} \int_{\Omega} c \theta w \widetilde{\zeta}^{\prime} d x d t+\int_{0}^{T} \int_{\Omega}(K \nabla \theta) \cdot \nabla w \widetilde{\zeta} d x d t \\
& =\int_{0}^{T} \int_{\Omega}\left(\mathcal{F}\left(\theta, \bar{v}+v^{0} \xi, D\left(\bar{v}+v^{0} \xi\right)\right): D\left(\bar{v}+v^{0} \xi\right)\right) w \widetilde{\zeta} d x d t \\
& \quad+\int_{0}^{T} \int_{\Omega} r(\theta) w \tilde{\zeta} d x d t+\int_{0}^{T} \int_{\omega} \theta^{b} w \tilde{\zeta} d x^{\prime} d t+\int_{\Omega} c \theta^{0} w \widetilde{\zeta}(0) d x \\
& \forall w \in W_{\Gamma_{1} \cup \Gamma_{L}}^{1, q^{\prime}}(\Omega), \forall \widetilde{\zeta} \in C^{\infty}([0, T]) \text { s.t. } \tilde{\zeta}(T)=0,
\end{aligned}
$$

where

$$
J:\left\{\begin{array}{l}
L^{p}\left(0, T ; V_{0}^{p}\right) \rightarrow \mathbb{R}, \\
\bar{\varphi} \mapsto \int_{0}^{T} \int_{\Gamma_{0}} k|\bar{\varphi}-\tilde{s}| d x d t, \quad \tilde{s}=s-\left(v^{0}\right)_{\tau} \xi
\end{array}\right.
$$

and $\langle\cdot, \cdot\rangle_{\mathcal{D}^{\prime}(0, T), \mathcal{D}(0, T)}$ and $(\cdot, \cdot)_{\mathbf{L}^{2}(\Omega)}$ (respectively $\left.(\cdot, \cdot)_{L^{2}(\Omega)}\right)$ denote the duality product between $\mathcal{D}(0, T)$ and $\mathcal{D}^{\prime}(0, T)$ and the inner product in $\mathbf{L}^{2}(\Omega)$ (respectively $L^{2}(\Omega)$ ).

For this non-linear fluid flow/heat transfer problem, a natural proof strategy applies a fixed point technique. Indeed, for any given temperature field $\theta \in L^{\tilde{q}}\left(0, T ; L^{q}(\Omega)\right)$ with $\tilde{q} \geq$ 1 and $q \geq 1$, the fluid flow problem (2.13)-(2.14) admits a solution [10,11]. Moreover, we know that parabolic problems with $L^{1}$ data given by

$$
\left\{\begin{array}{l}
\frac{\partial \theta}{\partial t}-\operatorname{div}(a(x, \nabla \theta))=g \quad \text { in } \Omega \times(0, T) \\
\theta=0 \quad \text { in } \partial \Omega \times(0, T)
\end{array}\right.
$$

with $g \in L^{1}\left(0, T ; L^{1}(\Omega)\right)$ and the coercivity property $a(x, \gamma) \cdot \gamma \geq \alpha_{a}\|\gamma\|^{2}\left(\alpha_{a}>0\right)$ for all $\gamma \in \mathbb{R}^{3}$ and for almost every $x \in \Omega$ admit a solution $\theta \in L^{q}\left(0, T ; W_{0}^{1, q}(\Omega)\right)$ with $1<q<\frac{5}{4}$ 
(see [6]). For a given fluid velocity $v=\bar{v}+v^{0} \xi \in L^{p}\left(0, T ; V_{\Gamma_{1}}^{p}\right)$, we can not apply this result directly to the heat transfer problem since we consider a nonconstant heat capacity and mixed Dirichlet-Neumann boundary conditions, but we may still expect the existence of a solution $\theta \in L^{q}\left(0, T ; W_{\Gamma_{1} \cup \Gamma_{L}}^{1, q}(\Omega)\right)$. Nevertheless, in both cases, the proofs of existence rely on compactness arguments and uniqueness of the solution to the flow problem (2.13)(2.14) for a given temperature is not ensured, nor the uniqueness of the solution to $L^{1}$ parabolic problems (2.16).

In order to cope with this difficulty, we will consider in Sect. 3 an auxiliary approximate fluid flow/heat transfer problem $\left(P_{\delta}\right)$, where the $L^{1}$ right-hand side in the heat equation (1.3) is replaced by a bounded one depending on a small parameter $0<\delta \ll 1$, and we will prove the existence of a solution $\left(\bar{v}_{\delta}, \pi_{\delta}, \theta_{\delta}\right)$ to $\left(P_{\delta}\right)$ by using a fixed point argument. Then in Sect. 4 , we will prove that the sequence $\left(\bar{v}_{\delta}, \pi_{\delta}, \theta_{\delta}\right)_{\delta>0}$ converges to a solution of problem $(P)$.

\section{The approximate problem $\left(P_{\delta}\right)$}

For any $\delta>0$, we consider the following approximate problem:

Problem $\left(P_{\delta}\right)$ Let $c, K, r$, and $\mu$ satisfy (2.3)-(2.10). Let $f \in L^{p^{\prime}}\left(0, T ; \mathbf{L}^{2}(\Omega)\right), k \in L^{p^{\prime}}(0, T$; $\left.L_{+}^{p^{\prime}}\left(\Gamma_{0}\right)\right), s \in L^{p}\left(0, T ; \mathbf{L}^{p}\left(\Gamma_{0}\right)\right), \xi \in W^{1, p^{\prime}}(0, T)$ satisfying $(1.6), \theta^{b} \in L^{1}((0, T) \times \omega), \theta^{0} \in L^{1}(\Omega)$ and $v^{0} \in \mathbf{W}^{1, p}(\Omega)$ satisfying $(2.12)$.

Find $\theta_{\delta} \in W^{1,2}((0, T) \times \Omega) \cap L^{\infty}\left(0, T ; W_{\Gamma_{1} \cup \Gamma_{L}}^{1,2}(\Omega)\right) \cap C^{0}\left([0, T] ; L^{2}(\Omega)\right), \bar{v}_{\delta} \in C([0, T]$; $\left.\mathbf{L}^{2}(\Omega)\right) \cap L^{p}\left(0, T ; V_{0 . \text { div }}^{p}\right)$ with $\frac{\partial \bar{v}_{\delta}}{\partial t} \in L^{p^{\prime}}\left(0, T ;\left(V_{0 \text {.div }}^{p}\right)^{\prime}\right)$ and $\pi_{\delta} \in H^{-1}\left(0, T ; L_{0}^{p^{\prime}}(\Omega)\right)$, satisfying the following parabolic variational coupled problem:

$$
\begin{aligned}
\left\langle\frac{\partial}{\partial t}\left(\bar{v}_{\delta}, \tilde{\vartheta}\right)_{\mathbf{L}^{2}(\Omega)}, \zeta\right\rangle_{\mathcal{D}^{\prime}(0, T), \mathcal{D}(0, T)} & \\
& +\int_{0}^{T} \int_{\Omega} \mathcal{F}\left(\theta_{\delta}, \bar{v}_{\delta}+v^{0} \xi, D\left(\bar{v}_{\delta}+v^{0} \xi\right)\right): D(\tilde{\vartheta}) \zeta d x d t \\
& -\left\langle\int_{\Omega} \pi_{\delta} \operatorname{div}(\tilde{\vartheta}) d x, \zeta\right\rangle_{\mathcal{D}^{\prime}(0, T), \mathcal{D}(0, T)}+J\left(\bar{v}_{\delta}+\tilde{\vartheta} \zeta\right)-J\left(\bar{v}_{\delta}\right) \\
\geq & \int_{0}^{T}\left(f+v^{0} \frac{\partial \xi}{\partial t}, \tilde{\vartheta}\right)_{\mathbf{L}^{2}(\Omega)} \zeta d t \quad \forall \tilde{\vartheta} \in V_{0}^{p}, \forall \zeta \in \mathcal{D}(0, T)
\end{aligned}
$$

and

$$
\begin{aligned}
\int_{0}^{T} & \int_{\Omega} c \frac{\partial \theta_{\delta}}{\partial t} w \widetilde{\zeta} d x d t+\int_{0}^{T} \int_{\Omega}\left(K \nabla \theta_{\delta}\right) \cdot \nabla w \widetilde{\zeta} d x d t \\
= & \int_{0}^{T} \int_{\Omega} g_{\delta}\left(\theta_{\delta}, \bar{v}_{\delta}\right) w \widetilde{\zeta} d x d t+\int_{0}^{T} \int_{\Omega} r\left(\theta_{\delta}\right) w \widetilde{\zeta} d x d t \\
& +\int_{0}^{T} \int_{\omega} \theta_{\delta}^{b} w \widetilde{\zeta} d x^{\prime} d t \quad \forall w \in W_{\Gamma_{1} \cup \Gamma_{L}}^{1,2}(\Omega), \forall \tilde{\zeta} \in \mathcal{D}(0, T)
\end{aligned}
$$

with the initial conditions

$$
\bar{v}_{\delta}(0, \cdot)=0 \quad \text { in } \Omega
$$


and

$$
\theta_{\delta}(0, \cdot)=\theta_{\delta}^{0} \quad \text { in } \Omega
$$

where

$$
g_{\delta}\left(\theta_{\delta}, \bar{v}_{\delta}\right)=\frac{2 \mu\left(\theta_{\delta}, \bar{v}_{\delta}+v^{0} \xi,\left|D\left(\bar{v}_{\delta}+v^{0} \xi\right)\right|\right)\left|D\left(\bar{v}_{\delta}+v^{0} \xi\right)\right|^{p}}{1+2 \delta \mu\left(\theta_{\delta}, \bar{v}_{\delta}+v^{0} \xi,\left|D\left(\bar{v}_{\delta}+v^{0} \xi\right)\right|\right)\left|D\left(\bar{v}_{\delta}+v^{0} \xi\right)\right|^{p}}
$$

and $\theta_{\delta}^{b}$ and $\theta_{\delta}^{0}$ are chosen as smooth approximations of $\theta^{b}$ and $\theta^{0}$, respectively, i.e., $\theta_{\delta}^{b} \in$ $\mathcal{D}((0, T) \times \omega)$ and $\theta_{\delta}^{0} \in \mathcal{D}(\Omega)$ such that

$$
\left\|\theta_{\delta}^{b}-\theta^{b}\right\|_{L^{1}((0, T) \times \omega)} \leq \delta, \quad\left\|\theta_{\delta}^{0}-\theta^{0}\right\|_{L^{1}(\Omega)} \leq \delta .
$$

In order to prove the existence of a solution to $\left(P_{\delta}\right)$, we apply a fixed point technique. As a first step, we consider the fully decoupled fluid flow and heat transfer problems $\left(P_{(\tilde{u}, \tilde{\theta})}^{\text {flow }}\right)$ and $\left(P_{\delta,(\tilde{u}, \tilde{\theta})}^{\text {heat }}\right)$ :

Problem $\left(P_{(\tilde{u}, \tilde{\theta})}^{\text {flow }}\right)$ Let $(\tilde{u}, \tilde{\theta})$ be given in $L^{p}\left(0, T ; \mathbf{L}^{p}(\Omega)\right) \times L^{\tilde{q}_{1}}\left(0, T ; L^{\tilde{q}_{2}}(\Omega)\right)$ with $\tilde{q}_{1}>1$ and $\tilde{q}_{2}>1$. Find $\bar{v}_{(\tilde{u}, \tilde{\theta})} \in C\left([0, T] ; \mathbf{L}^{2}(\Omega)\right) \cap L^{p}\left(0, T ; V_{0 . \operatorname{div}}^{p}\right)$ with $\frac{\partial \bar{v}_{(\tilde{u}, \tilde{\theta})}}{\partial t} \in L^{p^{\prime}}\left(0, T ;\left(V_{0 . \mathrm{div}}^{p}\right)^{\prime}\right)$ and $\pi_{(\tilde{u}, \tilde{\theta})} \in H^{-1}\left(0, T ; L_{0}^{p^{\prime}}(\Omega)\right)$, satisfying the following parabolic variational inequality

$$
\begin{aligned}
\left\langle\frac{\partial}{\partial t}\left(\bar{v}_{(\tilde{u}, \tilde{\theta})}, \tilde{\vartheta}\right)_{\mathbf{L}^{2}(\Omega)}, \zeta\right\rangle_{\mathcal{D}^{\prime}(0, T), \mathcal{D}(0, T)} & \\
& +\int_{0}^{T} \int_{\Omega} \mathcal{F}\left(\tilde{\theta}, \tilde{u}+v^{0} \xi, D\left(\bar{v}_{(\tilde{u}, \tilde{\theta})}+v^{0} \xi\right)\right): D(\tilde{\vartheta}) \zeta d x d t \\
& -\left\langle\int_{\Omega} \pi_{(\tilde{u}, \tilde{\theta})} \operatorname{div}(\tilde{\vartheta}) d x, \zeta\right\rangle_{\mathcal{D}^{\prime}(0, T), \mathcal{D}(0, T)}+J\left(\bar{v}_{(\tilde{u}, \tilde{\theta})}+\tilde{\vartheta} \zeta\right)-J\left(\bar{v}_{(\tilde{u}, \tilde{\theta})}\right) \\
\geq & \int_{0}^{T}\left(f+v^{0} \frac{\partial \xi}{\partial t}, \tilde{\vartheta}\right)_{\mathbf{L}^{2}(\Omega)} \zeta d t \quad \forall \tilde{\vartheta} \in V_{0}^{p}, \forall \zeta \in \mathcal{D}(0, T)
\end{aligned}
$$

with the initial condition

$$
\bar{v}_{(\tilde{u}, \tilde{\theta})}(0, \cdot)=0 \quad \text { in } \Omega
$$

and

Problem $\left(P_{\delta,(\tilde{u}, \tilde{\theta})}^{\text {heat }}\right)$ Let $(\tilde{u}, \tilde{\theta})$ be given in $L^{p}\left(0, T ; V_{0 \text {.div }}^{p}\right) \times L^{\tilde{q}_{1}}\left(0, T ; L^{\tilde{q}_{2}}(\Omega)\right)$ with $\tilde{q}_{1}>1$ and $\tilde{q}_{2}>1$. Find $\theta_{\delta,(\tilde{u}, \tilde{\theta})} \in W^{1,2}((0, T) \times \Omega) \cap L^{\infty}\left(0, T ; W_{\Gamma_{1} \cup \Gamma_{L}}^{1,2}(\Omega)\right) \cap C^{0}\left([0, T] ; L^{2}(\Omega)\right)$, satisfying the following parabolic variational equality:

$$
\begin{aligned}
\int_{0}^{T} & \int_{\Omega} c \frac{\partial \theta_{\delta,(\tilde{u}, \tilde{\theta})}}{\partial t} w \tilde{\zeta} d x d t+\int_{0}^{T} \int_{\Omega}\left(K \nabla \theta_{\delta,(\tilde{u}, \tilde{\theta})}\right) \cdot \nabla w \tilde{\zeta} d x d t \\
= & \int_{0}^{T} \int_{\Omega} g_{\delta}(\tilde{\theta}, \tilde{u}) w \tilde{\zeta} d x d t+\int_{0}^{T} \int_{\Omega} r(\tilde{\theta}) w \tilde{\zeta} d x d t \\
& +\int_{0}^{T} \int_{\omega} \theta_{\delta}^{b} w \widetilde{\zeta} d x^{\prime} d t \quad \forall w \in W_{\Gamma_{1} \cup \Gamma_{L}}^{1,2}(\Omega), \forall \tilde{\zeta} \in \mathcal{D}(0, T)
\end{aligned}
$$


with the initial condition

$$
\theta_{\delta,(\tilde{u}, \tilde{\theta})}(0, \cdot)=\theta_{\delta}^{0} \quad \text { in } \Omega
$$

Remark 3.1 Let us emphasize that problem $\left(P_{(\tilde{u}, \tilde{\theta})}^{\text {flow }}\right)$ does not depend on $\delta$.

Then we have

Proposition 3.2 (Existence and uniqueness result for $\left.\left(P_{(\tilde{u}, \tilde{\theta})}^{\text {flow }}\right)\right)$ Let $(\tilde{u}, \tilde{\theta})$ be given in $L^{p}\left(0, T ; \mathbf{L}^{p}(\Omega)\right) \times L^{\tilde{q}_{1}}\left(0, T ; L^{\tilde{q}_{2}}(\Omega)\right)$ with $\tilde{q}_{1}>1$ and $\tilde{q}_{2}>1$. Let $\mu$ satisfy (2.8)-(2.10), $f \in L^{p^{\prime}}\left(0, T ; \mathbf{L}^{2}(\Omega)\right), k \in L^{p^{\prime}}\left(0, T ; L_{+}^{p^{\prime}}\left(\Gamma_{0}\right)\right), s \in L^{p}\left(0, T ; \mathbf{L}^{p}\left(\Gamma_{0}\right)\right), \xi \in W^{1, p^{\prime}}(0, T)$ satisfying (1.6), and $v^{0} \in \mathbf{W}^{1, p}(\Omega)$ satisfying (2.12). Then problem $\left(P_{(\tilde{u}, \tilde{\theta})}^{\text {flow }}\right)$ admits a unique solution. Moreover, there exists a constant $C^{\text {flow }}$, independent of $(\tilde{\theta}, \tilde{u})$, such that

$$
\begin{aligned}
& \left\|\bar{v}_{(\tilde{u}, \tilde{\theta})}\right\|_{L^{p}\left(0, T ; V_{0 . \text { div }}^{p}\right)} \leq C^{\text {flow }}, \\
& \left\|\bar{v}_{(\tilde{u}, \tilde{\theta})}\right\|_{L^{\infty}\left(0, T ; \mathbf{L}^{2}(\Omega)\right)} \leq C^{\text {flow }}
\end{aligned}
$$

and

$$
\left\|\frac{\partial \bar{v}_{(\tilde{u}, \tilde{\theta})}}{\partial t}\right\|_{L^{p^{\prime}\left(0, T ;\left(V_{0 . \mathrm{div}}^{p}\right)^{\prime}\right)}} \leq C^{\text {flow }} .
$$

Proof The existence and uniqueness of a solution to the problem $\left(P_{(\tilde{u}, \tilde{\theta})}^{\text {flow }}\right)$ is an immediate consequence of Theorem 3.1 in [10] when $p \in[6 / 5,2)$ and Theorem 3.1, Theorem 4.1 and Remark 4.1 in [11] when $p \geq 2$. Let us prove now (3.11) and (3.13). For any $\bar{\varphi}=\tilde{\vartheta} \zeta$ with $\tilde{\vartheta} \in V_{0 . \operatorname{div}}^{p}$ and $\zeta \in \mathcal{D}(0, T)$, we have

$$
\begin{aligned}
& \underbrace{\left.\left\langle\frac{\partial}{\partial t}\left(\bar{v}_{(\tilde{u}, \tilde{\theta})}, \tilde{\vartheta}\right)_{\mathbf{L}^{2}(\Omega)}, \zeta\right\rangle_{\mathcal{D}^{\prime}(0, T), \mathcal{D}(0, T)}\right)^{\prime}, V_{0 . \mathrm{div}}^{p} d t}_{\quad=\int_{0}^{T}<\frac{\partial \bar{v}(\tilde{u}, \tilde{\theta})}{\partial t}, \bar{\varphi}>} \\
& \quad+\int_{0}^{T} \int_{\Omega} \mathcal{F}\left(\tilde{\theta}, \tilde{u}+v^{0} \xi, D\left(\bar{v}_{(\tilde{u}, \tilde{\theta})}+v^{0} \xi\right)\right): D(\bar{\varphi}) d x d t \\
& \quad+J\left(\bar{v}_{(\tilde{u}, \tilde{\theta})}+\bar{\varphi}\right)-J\left(\bar{v}_{(\tilde{u}, \tilde{\theta})}\right) \geq \int_{0}^{T}\left(f+v^{0} \frac{\partial \xi}{\partial t}, \bar{\varphi}\right)_{\mathbf{L}^{2}(\Omega)} d t .
\end{aligned}
$$

By density of $\mathcal{D}(0, T) \otimes V_{0 \text {.div }}^{p}$ into $L^{p}\left(0, T ; V_{0 \text {.div }}^{p}\right)$, the same inequality is true for any $\bar{\varphi} \in$ $L^{p}\left(0, T ; V_{0 \text {.div }}^{p}\right)$.

Let us choose $\bar{\varphi}=-\bar{v}_{(\tilde{u}, \tilde{\theta})} \mathbf{1}_{[[0, t]}$, where $t \in(0, T]$, and $\mathbf{1}_{[0, t]}$ is the indicatrix function of the time interval $[0, t]$. With $(3.14)$, we have

$$
\begin{aligned}
& \int_{0}^{t}\left\langle\frac{\left.\partial \bar{v}_{(\tilde{u}, \tilde{\theta})}, \bar{v}_{(\tilde{u}, \tilde{\theta})}\right\rangle_{\left(V_{0 . \mathrm{div}}^{p}\right)^{\prime}, V_{0 . \mathrm{div}}^{p}} d \tilde{t}}{\quad+\int_{0}^{t} \int_{\Omega} \mathcal{F}\left(\tilde{\theta}, \tilde{u}+v^{0} \xi, D\left(\bar{v}_{(\tilde{u}, \tilde{\theta})}+v^{0} \xi\right)\right): D\left(\bar{v}_{(\tilde{u}, \tilde{\theta})}\right) d x d \tilde{t}}\right. \\
& \leq \int_{0}^{t}\left(f+v^{0} \frac{\partial \xi}{\partial t}, \bar{v}_{(\tilde{u}, \tilde{\theta})}\right)_{\mathbf{L}^{2}(\Omega)} d \tilde{t}+\int_{0}^{t} \int_{\Gamma_{0}} k|\tilde{s}| d x d \tilde{t} .
\end{aligned}
$$


With (2.10) and (2.1)-(2.2), we get

$$
\begin{aligned}
\int_{0}^{t} & \int_{\Omega} \mathcal{F}\left(\tilde{\theta}, \tilde{u}+v^{0} \xi, D\left(\bar{v}_{(\tilde{u}, \tilde{\theta})}+v^{0} \xi\right)\right): D\left(\bar{v}_{(\tilde{u}, \tilde{\theta})}\right) d x d \tilde{t} \\
\geq & 2\left(C_{\text {Korn }, p}\right)^{p} \mu_{0} \int_{0}^{t}\left\|\bar{v}_{(\tilde{u}, \tilde{\theta})}+v^{0} \xi\right\|_{1 . p}^{p} d \tilde{t} \\
& -2 \mu_{1} \int_{0}^{t}\left\|v^{0} \xi\right\|_{1 . p}\left\|\bar{v}_{(\tilde{u}, \tilde{\theta})}+v^{0} \xi\right\|_{1 . p}^{p-1} d \tilde{t} \\
\geq & 2\left(C_{\text {Korn }, p}\right)^{p} \mu_{0}\left|\left\|\bar{v}_{(\tilde{u}, \tilde{\theta})}\right\|_{L^{p}\left(0, t ; V_{0 . \text { div }}^{p}\right)}-\left\|v^{0} \xi\right\|_{L^{p}\left(0, t ; V_{\Gamma_{1}}^{p}\right)}\right|^{p} \\
& -2 \mu_{1}\left(\left\|\bar{v}_{(\tilde{u}, \tilde{\theta})}\right\|_{L^{p}\left(0, t ; V_{0 . \text { div }}^{p}\right)}+\left\|v^{0} \xi\right\|_{L^{p}\left(0, t ; V_{\Gamma_{1}}^{p}\right)}\right)^{p-1}\left\|v^{0} \xi\right\|_{L^{p}\left(0, t ; V_{\Gamma_{1}}^{p}\right)} .
\end{aligned}
$$

Since $f+v^{0} \frac{\partial \xi}{\partial t} \in L^{p^{\prime}}\left(0, T ; \mathbf{L}^{2}(\Omega)\right)$, we obtain

$$
\begin{aligned}
& \frac{1}{2}\left\|\bar{v}_{(\tilde{u}, \tilde{\theta})}(t)\right\|_{\mathbf{L}^{2}(\Omega)}^{2} \\
& \quad+2\left(C_{\mathrm{Korn}, p}\right)^{p} \mu_{0}\left|\left\|\bar{v}_{(\tilde{u}, \tilde{\theta})}\right\|_{L^{p}\left(0, t ; V_{0 . \text { div }}^{p}\right)}-\left\|v^{0} \xi\right\|_{L^{p}\left(0, t ; V_{\Gamma_{1}}^{p}\right)}\right|^{p} \\
& \leq \widetilde{C}\left\|f+v^{0} \frac{\partial \xi}{\partial t}\right\|_{L^{p^{\prime}\left(0, t ; \mathbf{L}^{2}(\Omega)\right)}}\left\|\bar{v}_{(\tilde{u}, \tilde{\theta})}\right\|_{L^{p}\left(0, t ; V_{0 . \text { div }}^{p}\right)}+\int_{0}^{t} \int_{\Gamma_{0}} k|\tilde{s}| d x d \tilde{t} \\
& \quad+2 \mu_{1}\left(\left\|\bar{v}_{(\tilde{u}, \tilde{\theta})}\right\|_{L^{p}\left(0, t ; V_{0 . \text { div }}^{p}\right)}+\left\|v^{0} \xi\right\|_{L^{p}\left(0, t ; V_{\Gamma_{1}}^{p}\right)}\right)^{p-1}\left\|v^{0} \xi\right\|_{L^{p}\left(0, t ; V_{\Gamma_{1}}^{p}\right)},
\end{aligned}
$$

where $\widetilde{C}$ denotes the norm of the continuous injection of $V_{0}^{p}$ into $\mathbf{L}^{2}(\Omega)$.

Let us consider first $t=T$. If $\left\|\bar{v}_{(\tilde{u}, \tilde{\theta})}\right\|_{L^{p}\left(0, T ; V_{0 . \text { div }}^{p}\right)} \neq 0$, it follows that

$$
\begin{aligned}
& 2\left(C_{\text {Korn }, p}\right)^{p} \mu_{0}\left|1-\frac{\left\|v^{0} \xi\right\|_{L^{p}\left(0, T ; V_{\Gamma_{1}}^{p}\right)}}{\left\|\bar{v}_{(\tilde{u}, \tilde{\theta})}\right\|_{L^{p}\left(0, T ; V_{0 . \text { div }}^{p}\right)}}\right|^{p} \\
& \leq \widetilde{C}\left\|f+v^{0} \frac{\partial \xi}{\partial t}\right\|_{L^{p^{\prime}\left(0, T ; \mathbf{L}^{2}(\Omega)\right)}}\left\|\bar{v}_{(\tilde{u}, \tilde{\theta})}\right\|_{L^{p}\left(0, T ; V_{0 . \mathrm{div}}^{p}\right)}^{1-p}+\frac{J(0)}{\left\|\bar{v}_{(\tilde{u}, \tilde{\theta})}\right\|_{L^{p}\left(0, T ; V_{0 . \mathrm{div}}^{p}\right)}^{p}} \\
& +2 \mu_{1}\left(1+\frac{\left\|v^{0} \xi\right\|_{L^{p}\left(0, T ; V_{\Gamma_{1}}^{p}\right)}}{\left\|\bar{v}_{(\tilde{u}, \tilde{\theta})}\right\|_{L^{p}\left(0, T ; V_{0 . \text { div }}^{p}\right)}}\right)^{p-1} \frac{\left\|v^{0} \xi\right\|_{L^{p}\left(0, T ; V_{\Gamma_{1}}^{p}\right)}}{\left\|\bar{v}_{(\tilde{u}, \tilde{\theta})}\right\|_{L^{p}\left(0, T ; V_{0 . \text { div }}^{p}\right)}} .
\end{aligned}
$$

However, the mapping

$$
\begin{aligned}
& z \mapsto 2\left(C_{\text {Korn }, p}\right)^{p} \mu_{0}\left|1-\frac{\left\|v^{0} \xi\right\|_{L^{p}\left(0, T ; V_{\Gamma_{1}}^{p}\right)}}{z}\right|^{p}-\widetilde{C}\left\|f+v^{0} \frac{\partial \xi}{\partial t}\right\|_{L^{p^{\prime}\left(0, T ; \mathbf{L}^{2}(\Omega)\right)}} z^{1-p} \\
& -\frac{J(0)}{z^{p}}-2 \mu_{1}\left(1+\frac{\left\|v^{0} \xi\right\|_{L^{p}\left(0, T ; V_{\Gamma_{1}}^{p}\right)}}{z}\right)^{p-1} \frac{\left\|v^{0} \xi\right\|_{L^{p}\left(0, T ; V_{\Gamma_{1}}^{p}\right)}}{z}
\end{aligned}
$$

admits $2\left(C_{\mathrm{Korn}, p}\right)^{p} \mu_{0}>0$ as a limit when $z$ tends to $+\infty$. Thus, there exists a real number $C>0$, independent of $(\tilde{u}, \tilde{\theta})$, such that

$$
\left\|\bar{v}_{(\tilde{u}, \tilde{\theta})}\right\|_{L^{p}\left(0, T ; V_{0 . \text { div }}^{p}\right)} \leq C
$$

which yields (3.11). Going back to (3.15), we obtain (3.12). 
Let us choose now $\bar{\varphi}= \pm \tilde{\vartheta} \zeta$ with $\tilde{\vartheta} \in V_{0 \text { div }}^{p}$ and $\zeta \in \mathcal{D}(0, T)$ in (3.14). We obtain

$$
\begin{aligned}
& \int_{0}^{T}\left\langle\frac{\partial \bar{v}_{(\tilde{u}, \tilde{\theta})}}{\partial t}, \pm\left.\tilde{\vartheta} \zeta\right|_{\left(V_{0 . \mathrm{div}}^{p}{ }^{\prime}, V_{0 . \mathrm{div}}^{p}\right.} d t\right. \\
& \quad+\int_{0}^{T} \int_{\Omega} \mathcal{F}\left(\tilde{\theta}, \tilde{u}+v^{0} \xi, D\left(\bar{v}_{(\tilde{u}, \tilde{\theta})}+v^{0} \xi\right)\right): D( \pm \tilde{\vartheta} \zeta) d x d t \\
& \quad+J\left(\bar{v}_{(\tilde{u}, \tilde{\theta})} \pm \tilde{\vartheta} \zeta\right)-J\left(\bar{v}_{(\tilde{u}, \tilde{\theta})}\right) \geq \int_{0}^{T}\left(f+v^{0} \frac{\partial \xi}{\partial t}, \pm \tilde{\vartheta} \zeta\right)_{\mathbf{L}^{2}(\Omega)} d t
\end{aligned}
$$

But

$$
\left|J\left(\bar{v}_{(\tilde{u}, \tilde{\theta})} \pm \tilde{\vartheta} \zeta\right)-J\left(\bar{v}_{(\tilde{u}, \tilde{\theta})}\right)\right| \leq \int_{0}^{T} \int_{\Gamma_{0}} k|\tilde{\vartheta} \zeta| d x d t
$$

and, recalling that $k \in L^{p^{\prime}}\left(0, T ; L_{+}^{p^{\prime}}\left(\Gamma_{0}\right)\right)$, we get

$$
\begin{aligned}
& \left|J\left(\bar{v}_{(\tilde{u}, \tilde{\theta})} \pm \tilde{\vartheta} \zeta\right)-J\left(\bar{v}_{(\tilde{u}, \tilde{\theta})}\right)\right| \\
& \quad \leq\left\|\gamma_{p}\right\|_{\mathcal{L}\left(\mathbf{W}^{1, p}(\Omega), \mathbf{L}^{p}(\partial \Omega)\right)}\|k\|_{L^{p^{\prime}}\left(0, T ; L^{p^{\prime}}\left(\Gamma_{0}\right)\right)}\|\tilde{\vartheta} \zeta\|_{L^{p}\left(0, T ; V_{0 . \text { div }}^{p}\right)},
\end{aligned}
$$

where $\gamma_{p}$ denotes the trace operator from $\mathbf{W}^{1, p}(\Omega)$ into $\mathbf{L}^{p}(\partial \Omega)$.

On the other hand,

$$
\begin{aligned}
& \left|\int_{0}^{T} \int_{\Omega} \mathcal{F}\left(\tilde{\theta}, \tilde{u}+v^{0} \xi, D\left(\bar{v}_{(\tilde{u}, \tilde{\theta})}+v^{0} \xi\right)\right): D(\tilde{\vartheta} \zeta) d x d t\right| \\
& \quad \leq 2 \mu_{1}\left\|D\left(\bar{v}_{(\tilde{u}, \tilde{\theta})}+v^{0} \xi\right)\right\|_{L^{p}\left(0, T ;\left(L^{p}(\Omega)\right)^{3 \times 3)}\right.}^{p-1}\|D(\tilde{\vartheta} \zeta)\|_{L^{p}\left(0, T ;\left(L^{p}(\Omega)\right)^{3 \times 3}\right)}
\end{aligned}
$$

and with (3.16), we obtain

$$
\begin{aligned}
& \left|\int_{0}^{T} \int_{\Omega} \mathcal{F}\left(\tilde{\theta}, \tilde{u}+v^{0} \xi, D\left(\bar{v}_{(\tilde{u}, \tilde{\theta})}+v^{0} \xi\right)\right): D(\tilde{\vartheta} \zeta) d x d t\right| \\
& \quad \leq\left(2 \mu_{1}\left(C+\left\|v^{0} \xi\right\|_{L^{p}\left(0, T ; V_{\Gamma_{1}}^{p}\right)}\right)^{p-1}\right)\|\tilde{\vartheta} \zeta\|_{L^{p}\left(0, T ; V_{0 . \text { div }}^{p}\right.} .
\end{aligned}
$$

Going back to (3.17), we obtain

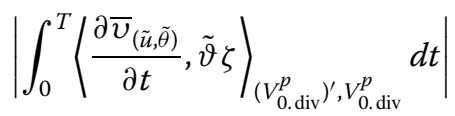

$$
\begin{aligned}
& \leq\left\|\gamma_{p}\right\|_{\mathcal{L}\left(\mathbf{W}^{1, p}(\Omega), \mathbf{L}^{p}(\partial \Omega)\right)}\|k\|_{L^{p^{\prime}\left(0, T ; L^{\prime}\left(\Gamma_{0}\right)\right)}}\|\tilde{\vartheta} \zeta\|_{L^{p}\left(0, T ; V_{0 . \text { div }}^{p}\right)} \\
& +\widetilde{C}\left\|f+v^{0} \frac{\partial \xi}{\partial t}\right\|_{L^{p^{\prime}\left(0, T ; \mathbf{L}^{2}(\Omega)\right)}}\|\tilde{\vartheta} \zeta\|_{L^{p}\left(0, T ; V_{0 . \text { div }}^{p}\right)} \\
& +\left(2 \mu_{1}\left(C+\left\|v^{0} \xi\right\|_{L^{p}\left(0, T ; V_{\Gamma_{1}}^{p}\right)}\right)^{p-1}\right)\|\tilde{\vartheta} \zeta\|_{L^{p}\left(0, T ; V_{0, \text { div }}^{p}\right.},
\end{aligned}
$$

which yields (3.13). 
Proposition 3.3 (Existence and uniqueness result for $\left.\left(P_{\delta, \tilde{u}, \tilde{\theta})}^{\text {heat }}\right)\right)$ Let $(\tilde{u}, \tilde{\theta})$ be given in $L^{p}\left(0, T ; V_{0 . \text { div }}^{p}\right) \times L^{\tilde{q}_{1}}\left(0, T ; L^{\tilde{q}_{2}}(\Omega)\right)$ with $\tilde{q}_{1}>1$ and $\tilde{q}_{2}>1$. Let $c, K$, $r$, and $\mu$ satisfy $(2.3)-$ (2.10). Let $\xi \in W^{1, p^{\prime}}(0, T)$ satisfying $(1.6), \theta_{\delta}^{b} \in \mathcal{D}((0, T) \times \omega), \theta_{\delta}^{0} \in \mathcal{D}(\Omega)$ and $v^{0} \in \mathbf{W}^{1, p}(\Omega)$ satisfying (2.12). Then problem $\left(P_{\delta, \tilde{u}, \tilde{\theta})}^{\text {heat }}\right)$ admits a unique solution. Moreover, there exists a constant $C^{\text {heat }}$, depending only on the data $K$ and c, such that

$$
\begin{aligned}
& \left\|\frac{\partial \theta_{\delta,(\tilde{u}, \tilde{\theta})}}{\partial t}\right\|_{L^{2}\left(0, T ; L^{2}(\Omega)\right)}^{2}+\left\|\nabla \theta_{\delta, \tilde{u}, \tilde{\theta})}\right\|_{L^{\infty}\left(0, T ; L^{2}(\Omega)\right)}^{2} \\
& \quad \leq C^{\text {heat }}\left(\left\|g_{\delta}(\tilde{\theta}, \tilde{u})+r(\tilde{\theta})\right\|_{L^{2}\left(0, T ; L^{2}(\Omega)\right)}^{2}+\left\|\theta_{\delta}^{0}\right\|_{H^{1}(\Omega)}^{2}+\left\|\theta_{\delta}^{b}\right\|_{W^{1,2}\left(0, T ; L^{2}(\omega)\right)}^{2}\right) .
\end{aligned}
$$

Proof The result is straightforward with the Galerkin method. The details are left to the reader.

Owing to the definition of $g_{\delta}(\tilde{\theta}, \tilde{u})$ and the uniform boundedness of the mapping $r$, we obtain immediately

Corollary 3.4 Under the previous assumptions, there exists a constant $C_{\delta}^{\text {heat }}$, depending only on $\delta,\left\|\theta_{\delta}^{0}\right\|_{H^{1}(\Omega)}$ and $\left\|\theta_{\delta}^{b}\right\|_{W^{1,2}\left(0, T ; L^{2}(\omega)\right)}$ such that

$$
\left\|\frac{\partial \theta_{\delta,(\tilde{u}, \tilde{\theta})}}{\partial t}\right\|_{L^{2}\left(0, T ; L^{2}(\Omega)\right)}+\left\|\nabla \theta_{\delta,(\tilde{u}, \tilde{\theta})}\right\|_{L^{\infty}\left(0, T ; L^{2}(\Omega)\right)} \leq C_{\delta}^{\text {heat }} .
$$

Proof By using (2.7) and (3.5), we obtain

$$
\begin{aligned}
& \left\|g_{\delta}(\tilde{\theta}, \tilde{u})+r(\tilde{\theta})\right\|_{L^{2}\left(0, T ; L^{2}(\Omega)\right)}^{2} \\
& \quad \leq 2 \int_{0}^{T} \int_{\Omega}\left(g_{\delta}(\tilde{\theta}, \tilde{u})\right)^{2} d x d t+2 \int_{0}^{T} \int_{\Omega}(r(\tilde{\theta}))^{2} d x d t \\
& \quad \leq 2\left(\frac{1}{\delta^{2}}+r_{1}^{2}\right) \operatorname{meas}(\Omega) T,
\end{aligned}
$$

which allows us to conclude.

It follows that, for any $(\tilde{u}, \tilde{\theta}) \in L^{p}\left(0, T ; \mathbf{L}^{p}(\Omega)\right) \times L^{2}\left(0, T ; L^{2}(\Omega)\right)$, and for any $\delta>0$, we may define $\left(\bar{v}_{(\tilde{u}, \tilde{\theta})}, \pi_{(\tilde{u}, \tilde{\theta})}\right) \in L^{p}\left(0, T ; V_{0 . \text { div }}^{p}\right) \times L^{p^{\prime}}\left(0, T ; L_{0}^{p^{\prime}}(\Omega)\right)$ as the unique solution of $\left(P_{(\tilde{u}, \tilde{\theta})}^{\text {flow }}\right)$

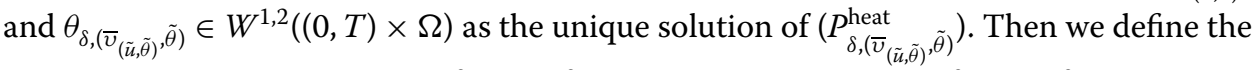
mapping $T_{\delta}: L^{p}\left(0, T ; \mathbf{L}^{p}(\Omega)\right) \times L^{2}\left(0, T ; L^{2}(\Omega)\right) \rightarrow L^{p}\left(0, T ; \mathbf{L}^{p}(\Omega)\right) \times L^{2}\left(0, T ; L^{2}(\Omega)\right)$ by

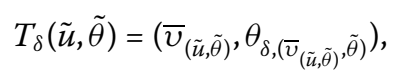

and we will prove that $T_{\delta}$ admits a fixed point.

Theorem 3.5 Let $\delta>0$. Let the mappings $c, K, r$, and $\mu$ satisfy (2.3)-(2.10), $f \in$ $L^{p^{\prime}}\left(0, T ; \mathbf{L}^{2}(\Omega)\right), k \in L^{p^{\prime}}\left(0, T ; L_{+}^{p^{\prime}}\left(\Gamma_{0}\right)\right), s \in L^{p}\left(0, T ; \mathbf{L}^{p}\left(\Gamma_{0}\right)\right), \xi \in W^{1, p^{\prime}}(0, T)$ satisfying (1.6), $\theta_{\delta}^{0} \in \mathcal{D}(\Omega), \theta_{\delta}^{b} \in \mathcal{D}((0, T) \times \omega)$ and $v^{0} \in \mathbf{W}^{1, p}(\Omega)$ satisfying (2.12). Then the mapping $T_{\delta}$ admits a fixed point in $L^{p}\left(0, T ; \mathbf{L}^{p}(\Omega)\right) \times L^{2}\left(0, T ; L^{2}(\Omega)\right)$. 
Proof Let $(\tilde{u}, \tilde{\theta}) \in L^{p}\left(0, T ; \mathbf{L}^{p}(\Omega)\right) \times L^{2}\left(0, T ; L^{2}(\Omega)\right)$. With the previous estimates, we have

$$
\left\|\bar{v}_{(\tilde{u}, \tilde{\theta})}\right\|_{L^{p}\left(0, T ; V_{0 . \text { div }}^{p}\right)} \leq C^{\text {flow }}, \quad\left\|\theta_{\delta,(\bar{v}}(\tilde{\tilde{u}, \tilde{\theta})}, \tilde{\theta})\right\|_{L^{\infty}\left(0, T ; W_{\Gamma_{1} \cup \Gamma_{L}}^{1,2}(\Omega)\right)} \leq C_{\delta}^{\text {heat }}
$$

Thus, $T_{\delta}(\mathcal{C}) \subset \mathcal{C}$ with $\mathcal{C}=\bar{B}_{L^{p}\left(0, T: L^{p}(\Omega)\right)}\left(0, C_{p} C^{\text {flow }}\right) \times \bar{B}_{L^{2}\left(0, T ; L^{2}(\Omega)\right.}\left(0, \sqrt{T} C_{2} C_{\delta}^{\text {heat }}\right)$, where $C_{p}$ and $C_{2}$ denote Poincaré's constant on $V_{0 \text {.div }}^{p}$ and on $W_{\Gamma_{1} \cup \Gamma_{L}}^{1,2}(\Omega)$, respectively. Moreover, the estimates (3.13) and (3.18) imply that $T_{\delta}(\mathcal{C})$ is bounded in the space $W^{1, p^{\prime}}\left(0, T ;\left(V_{0 \text {.div }}^{p}\right)^{\prime}\right) \times$ $W^{1,2}\left(0, T ; L^{2}(\Omega)\right)$, and we may conclude that $T_{\delta}(\mathcal{C})$ is relatively compact in $L^{p}\left(0, T ; \mathbf{L}^{p}(\Omega)\right) \times$ $L^{2}\left(0, T ; L^{2}(\Omega)\right)$.

Let us now prove that $T_{\delta}$ is continuous. Let $\left(\tilde{u}_{n}, \tilde{\theta}_{n}\right)_{n \geq 1}$ be a sequence, which converges strongly in $L^{p}\left(0, T ; \mathbf{L}^{p}(\Omega)\right) \times L^{2}\left(0, T ; L^{2}(\Omega)\right)$ to $(\tilde{u}, \tilde{\theta})$, and let us define

$$
T_{\delta}\left(\tilde{u}_{n}, \tilde{\theta}_{n}\right)=\left(\bar{v}_{\left(\tilde{u}_{n}, \tilde{\theta}_{n}\right)}, \theta_{\delta,\left(\bar{v}_{\left(\tilde{u}_{n}, \tilde{\theta}_{n}\right)}, \tilde{\theta}_{n}\right)}\right) \quad \text { for all } n \geq 1
$$

and

$$
T_{\delta}(\tilde{u}, \tilde{\theta})=\left(\bar{v}_{(\tilde{u}, \tilde{\theta})}, \theta_{\delta,(\bar{v}(\tilde{u}, \tilde{\theta}), \tilde{\theta})}\right)
$$

We have to prove that the sequence $\left(\bar{v}_{\left(\tilde{u}_{n}, \tilde{\theta}_{n}\right)}, \theta_{\delta,\left(\bar{v}_{\left(\tilde{u}_{n}, \tilde{\theta}_{n}\right)}, \tilde{\theta}_{n}\right)}\right)_{n \geq 1}$ converges strongly to $\left(\bar{v}_{(\tilde{u}, \tilde{\theta})}\right.$, $\left.\theta_{\left(\bar{v}_{\delta,(\tilde{u}, \tilde{\theta})}, \tilde{\theta}\right)}\right)$ in $L^{p}\left(0, T ; \mathbf{L}^{p}(\Omega)\right) \times L^{2}\left(0, T ; L^{2}(\Omega)\right)$.

The sequence $\left(\bar{v}_{\left(\tilde{u}_{n}, \tilde{\theta}_{n}\right)}, \theta_{\delta,\left(\bar{v}_{\left(\tilde{u}_{n}, \tilde{\theta}_{n}\right)}, \tilde{\theta}_{n}\right)}\right)_{n \geq 1}$ satisfies the estimates (3.11)-(3.13) and (3.18), so it admits strongly converging subsequences in $L^{p}\left(0, T ; \mathbf{L}^{p}(\Omega)\right) \times L^{2}\left(0, T ; L^{2}(\Omega)\right)$.

Let us consider such a subsequence still denoted $\left(\bar{v}_{\left(\tilde{u}_{n}, \tilde{\theta}_{n}\right)}, \theta_{\delta,\left(\bar{v}_{\left(\tilde{u}_{n}, \tilde{\theta}_{n}\right)} \tilde{\theta}_{n}\right)}\right)_{n \geq 1}$.

With (3.14), we have

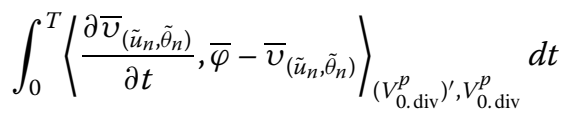

$$
\begin{aligned}
& +\int_{0}^{T} \int_{\Omega} \mathcal{F}\left(\tilde{\theta}_{n}, \tilde{u}_{n}+v^{0} \xi, D\left(\bar{v}_{\left(\tilde{u}_{n}, \tilde{\theta}_{n}\right)}+v^{0} \xi\right)\right): D\left(\bar{\varphi}-\bar{v}_{\left(\tilde{u}_{n}, \tilde{\theta}_{n}\right)}\right) d x d t \\
& +J(\bar{\varphi})-J\left(\bar{v}_{\left(\tilde{u}_{n}, \tilde{\theta}_{n}\right)}\right) \\
& \geq \int_{0}^{T}\left(f+v^{0} \frac{\partial \xi}{\partial t}, \bar{\varphi}-\bar{v}_{\left(\tilde{u}_{n}, \tilde{\theta}_{n}\right)}\right)_{\mathbf{L}^{2}(\Omega)} d t \quad \forall \bar{\varphi} \in L^{p}\left(0, T ; V_{0 . \text { div }}^{p}\right)
\end{aligned}
$$

for all $n \geq 1$, we also have

$$
\begin{aligned}
& \int_{0}^{T}\left\langle\frac{\partial \bar{v}_{(\tilde{u}, \tilde{\theta})}}{\partial t}, \bar{\varphi}-\bar{v}_{(\tilde{u}, \tilde{\theta})}\right\rangle_{\left(V_{0 . \text { div }}^{p}\right)^{\prime}, V_{0 . \text { div }}^{p}} d t \\
& +\int_{0}^{T} \int_{\Omega} \mathcal{F}\left(\tilde{\theta}, \tilde{u}+v^{0} \xi, D\left(\bar{v}_{(\tilde{u}, \tilde{\theta})}+v^{0} \xi\right)\right): D\left(\bar{\varphi}-\bar{v}_{(\tilde{u}, \tilde{\theta})}\right) d x d t \\
& +J(\bar{\varphi})-J\left(\bar{v}_{(\tilde{u}, \tilde{\theta})}\right) \\
& \geq \int_{0}^{T}\left(f+v^{0} \frac{\partial \xi}{\partial t}, \bar{\varphi}-\bar{v}_{(\tilde{u}, \tilde{\theta})}\right)_{\mathbf{L}^{2}(\Omega)} d t \quad \forall \bar{\varphi} \in L^{p}\left(0, T ; V_{0 . \operatorname{div}}^{p}\right) .
\end{aligned}
$$


We choose $\bar{\varphi}=\bar{v}_{(\tilde{u}, \tilde{\theta})}$ in (3.19) then $\bar{\varphi}=\bar{v}_{\left(\tilde{u}_{n}, \tilde{\theta}_{n}\right)}$ in (3.20) and add the two inequalities. We obtain:

$$
\begin{aligned}
& \int_{0}^{T}\left\langle\frac{\partial\left(\bar{v}_{\left(\tilde{u}_{n}, \tilde{\theta}_{n}\right)}-\bar{v}_{(\tilde{u}, \tilde{\theta})}\right)}{\partial t}, \bar{v}_{\left(\tilde{u}_{n}, \tilde{\theta}_{n}\right)}-\left.\bar{v}_{(\tilde{u}, \tilde{\theta})}\right|_{\left(V_{0 . \mathrm{div}}^{p}\right)^{\prime}, V_{0 . \mathrm{div}}^{p}} d t\right. \\
& \quad+\int_{0}^{T} \int_{\Omega}\left(\mathcal{F}\left(\tilde{\theta}_{n}, \tilde{u}_{n}+v^{0} \xi, D\left(\bar{v}_{\left(\tilde{u}_{n}, \tilde{\theta}_{n}\right)}+v^{0} \xi\right)\right)\right. \\
& \left.\quad-\mathcal{F}\left(\tilde{\theta}, \tilde{u}+v^{0} \xi, D\left(\bar{v}_{(\tilde{u}, \tilde{\theta})}+v^{0} \xi\right)\right)\right): D\left(\bar{v}_{\left(\tilde{u}_{n}, \tilde{\theta}_{n}\right)}-\bar{v}_{(\tilde{u}, \tilde{\theta})}\right) d x d t \leq 0
\end{aligned}
$$

and thus,

$$
\begin{aligned}
& \frac{1}{2}\left\|\bar{v}_{\left(\tilde{u}_{n}, \tilde{\theta}_{n}\right)}-\bar{v}_{(\tilde{u}, \tilde{\theta})}\right\|_{\mathbf{L}^{2}(\Omega)}^{2}(T) \\
& \quad+\int_{0}^{T} \int_{\Omega}\left(\mathcal{F}\left(\tilde{\theta}_{n}, \tilde{u}_{n}+v^{0} \xi, D\left(\bar{v}_{\left(\tilde{u}_{n}, \tilde{\theta}_{n}\right)}+v^{0} \xi\right)\right)\right. \\
& \left.\quad-\mathcal{F}\left(\tilde{\theta}_{n}, \tilde{u}_{n}+v^{0} \xi, D\left(\bar{v}_{(\tilde{u}, \tilde{\theta})}+v^{0} \xi\right)\right)\right): D\left(\bar{v}_{\left(\tilde{u}_{n}, \tilde{\theta}_{n}\right)}-\bar{v}_{(\tilde{u}, \tilde{\theta})}\right) d x d t \\
& \leq \int_{0}^{T} \int_{\Omega}\left(\mathcal{F}\left(\tilde{\theta}, \tilde{u}+v^{0} \xi, D\left(\bar{v}_{(\tilde{u}, \tilde{\theta})}+v^{0} \xi\right)\right)\right. \\
& \left.\quad-\mathcal{F}\left(\tilde{\theta}_{n}, \tilde{u}_{n}+v^{0} \xi, D\left(\bar{v}_{(\tilde{u}, \tilde{\theta})}+v^{0} \xi\right)\right)\right): D\left(\bar{v}_{\left(\tilde{u}_{n}, \tilde{\theta}_{n}\right)}-\bar{v}_{(\tilde{u}, \tilde{\theta})}\right) d x d t .
\end{aligned}
$$

We decompose $\mathcal{F}$ as $\mathcal{F}=\mathcal{F}_{1}+\mathcal{F}_{2}$ with

$$
\mathcal{F}_{1}\left(\lambda_{2}\right)=\mu_{0}\left\|\lambda_{2}\right\|^{p-2} \lambda_{2} \quad \text { if } \lambda_{2} \neq 0_{\mathbb{R}^{3 \times 3}}, \quad \mathcal{F}_{1}\left(\lambda_{2}\right)=0_{\mathbb{R}^{3 \times 3}} \quad \text { otherwise }
$$

and

$$
\left\{\begin{array}{l}
\mathcal{F}_{2}\left(\lambda_{0}, \lambda_{1}, \lambda_{2}\right)=2 \bar{\mu}\left(\lambda_{0}, \lambda_{1},\left\|\lambda_{2}\right\|\right)\left\|\lambda_{2}\right\|^{p-2} \lambda_{2} \quad \text { if } \lambda_{2} \neq 0_{\mathbb{R}^{3 \times 3}}, \\
\mathcal{F}_{2}\left(\lambda_{0}, \lambda_{1}, \lambda_{2}\right)=0_{\mathbb{R}^{3 \times 3}} \quad \text { otherwise, }
\end{array}\right.
$$

where $\bar{\mu}=\mu-\frac{\mu_{0}}{2}$. By observing that $\bar{\mu}$ satisfies

$$
\begin{aligned}
& d \mapsto \bar{\mu}(\cdot, \cdot, d) \text { is monotone increasing on } \mathbb{R}_{+}, \\
& 0<\frac{\mu_{0}}{2} \leq \bar{\mu}(o, e, d) \leq \mu_{1}-\frac{\mu_{0}}{2} \quad \text { for all }(o, e, d) \in \mathbb{R} \times \mathbb{R}^{3} \times \mathbb{R}_{+},
\end{aligned}
$$

we infer from Lemma 1 in [9] that $\lambda_{2} \mapsto \mathcal{F}_{2}\left(\cdot, \cdot, \lambda_{2}\right)$ is monotone in $\mathbb{R}^{3 \times 3}$. Hence,

$$
\begin{aligned}
& \int_{0}^{T} \int_{\Omega}\left(\mathcal{F}_{2}\left(\tilde{\theta}_{n}, \tilde{u}_{n}+v^{0} \xi, D\left(\bar{v}_{\left(\tilde{u}_{n}, \tilde{\theta}_{n}\right)}+v^{0} \xi\right)\right)\right. \\
& \left.\quad-\mathcal{F}_{2}\left(\tilde{\theta}_{n}, \tilde{u}_{n}+v^{0} \xi, D\left(\bar{v}_{(\tilde{u}, \tilde{\theta})}+v^{0} \xi\right)\right)\right): D\left(\bar{v}_{\left(\tilde{u}_{n}, \tilde{\theta}_{n}\right)}-\bar{v}_{(\tilde{u}, \tilde{\theta})}\right) d x d t \geq 0
\end{aligned}
$$


and thus,

$$
\begin{aligned}
& \frac{1}{2}\left\|\bar{v}_{\left(\tilde{u}_{n}, \tilde{\theta}_{n}\right)}-\bar{v}_{(\tilde{u}, \tilde{\theta})}\right\|_{\mathbf{L}^{2}(\Omega)}^{2}(T) \\
& \quad+\int_{0}^{T} \int_{\Omega}\left(\mathcal{F}_{1}\left(D\left(\bar{v}_{\left(\tilde{u}_{n}, \tilde{\theta}_{n}\right)}+v^{0} \xi\right)\right)-\mathcal{F}_{1}\left(D\left(\bar{v}_{(\tilde{u}, \tilde{\theta})}+v^{0} \xi\right)\right)\right) \\
& \quad: D\left(\bar{v}_{\left(\tilde{u}_{n}, \tilde{\theta}_{n}\right)}-\bar{v}_{(\tilde{u}, \tilde{\theta})}\right) d x d t \\
& \leq \int_{0}^{T} \int_{\Omega}\left(\mathcal{F}_{2}\left(\tilde{\theta}, \tilde{u}+v^{0} \xi, D\left(\bar{v}_{(\tilde{u}, \tilde{\theta})}+v^{0} \xi\right)\right)\right. \\
& \left.\quad-\mathcal{F}_{2}\left(\tilde{\theta}_{n}, \tilde{u}_{n}+v^{0} \xi, D\left(\bar{v}_{(\tilde{u}, \tilde{\theta})}+v^{0} \xi\right)\right)\right): D\left(\bar{v}_{\left(\tilde{u}_{n}, \tilde{\theta}_{n}\right)}-\bar{v}_{(\tilde{u}, \tilde{\theta})}\right) d x d t .
\end{aligned}
$$

\section{Now we distinguish two cases.}

Case 1: $p \in[6 / 5,2)$

With some algebraic computations, we can prove that

$$
\left(\|\lambda\|+\left\|\lambda^{\prime}\right\|\right)^{2-p}\left(\mathcal{F}_{1}(\lambda)-\mathcal{F}_{1}\left(\lambda^{\prime}\right)\right):\left(\lambda-\lambda^{\prime}\right) \geq \mu_{0}(p-1)\left\|\lambda-\lambda^{\prime}\right\|^{2},
$$

which yields

$$
\left(\|\lambda\|^{p}+\left\|\lambda^{\prime}\right\|^{p}\right)^{\frac{2-p}{2}}\left(\left(\mathcal{F}_{1}(\lambda)-\mathcal{F}_{1}\left(\lambda^{\prime}\right)\right):\left(\lambda-\lambda^{\prime}\right)\right)^{\frac{p}{2}} \geq \frac{\left(\mu_{0}(p-1)\right)^{\frac{p}{2}}}{2^{\frac{(p-1)(2-p)}{2}}}\left\|\lambda-\lambda^{\prime}\right\|^{p}
$$

for all $\left(\lambda, \lambda^{\prime}\right) \in \mathbb{R}^{3 \times 3} \times \mathbb{R}^{3 \times 3}$ (see, for instance, Theorem 4.1 in [10]).

It follows that

$$
\begin{aligned}
& \frac{\left(\mu_{0}(p-1)\right)^{\frac{p}{2}}}{2 \frac{(p-1)(2-p)}{2}} \int_{0}^{T} \int_{\Omega}\left\|D\left(\bar{v}_{\left(\tilde{u}_{n}, \tilde{\theta}_{n}\right)}-\bar{v}_{(\tilde{u}, \tilde{\theta})}\right)\right\|^{p} d x d t \\
& \leq\left(\int_{0}^{T} \int_{\Omega}\left(\mathcal{F}_{1}\left(D\left(\bar{v}_{\left(\tilde{u}_{n}, \tilde{\theta}_{n}\right)}+v^{0} \xi\right)\right)-\mathcal{F}_{1}\left(D\left(\bar{v}_{(\tilde{u}, \tilde{\theta})}+v^{0} \xi\right)\right)\right)\right. \\
& \left.\quad: D\left(\bar{v}_{\left(\tilde{u}_{n}, \tilde{\theta}_{n}\right)}-\bar{v}_{(\tilde{u}, \tilde{\theta})}\right) d x d t\right)^{\frac{p}{2}} \\
& \quad \times\left(\int_{0}^{T} \int_{\Omega}\left(\left\|D\left(\bar{v}_{\left(\tilde{u}_{n}, \tilde{\theta}_{n}\right)}+v^{0} \xi\right)\right\|^{p}+\left\|D\left(\bar{v}_{(\tilde{u}, \tilde{\theta})}+v^{0} \xi\right)\right\|^{p}\right) d x d t\right)^{\frac{2-p}{2}} \\
& \leq\left(\int_{0}^{T} \int_{\Omega}\left(\mathcal{F}_{1}\left(D\left(\bar{v}_{\left(\tilde{u}_{n}, \tilde{\theta}_{n}\right)}+v^{0} \xi\right)\right)-\mathcal{F}_{1}\left(D\left(\bar{v}_{(\tilde{u}, \tilde{\theta})}+v^{0} \xi\right)\right)\right)\right. \\
& \left.\quad: D\left(\bar{v}_{\left(\tilde{u}_{n}, \tilde{\theta}_{n}\right)}-\bar{v}_{(\tilde{u}, \tilde{\theta})}\right) d x d t\right)^{\frac{p}{2}} \\
& \quad \times\left(\left\|\bar{v}_{\left(\tilde{u}_{n}, \tilde{\theta}_{n}\right)}+v^{0} \xi\right\|_{L^{p}\left(0, T ; V_{\Gamma_{1}}^{p}\right)}^{p}+\left\|\bar{v}_{(\tilde{u}, \tilde{\theta})}+v^{0} \xi\right\|_{L^{p}\left(0, T ; V_{\Gamma_{1}}^{p}\right)}^{p}\right)^{\frac{2-p}{2}} .
\end{aligned}
$$


Thus, with (3.11), we get

$$
\begin{aligned}
& \frac{\left(\mu_{0}(p-1)\right)^{\frac{p}{2}}}{2^{\frac{(p-1)(2-p)}{2}}}\left\|D\left(\bar{v}_{\left(\tilde{u}_{n}, \tilde{\theta}_{n}\right)}-\bar{v}_{(\tilde{u}, \tilde{\theta})}\right)\right\|_{L^{p}\left(0, T ;\left(L^{p}(\Omega)\right)^{3 \times 3}\right)}^{p} \\
& \leq\left(\int_{0}^{T} \int_{\Omega}\left(\mathcal{F}_{1}\left(D\left(\bar{v}_{\left(\tilde{u}_{n}, \tilde{\theta}_{n}\right)}+v^{0} \xi\right)\right)-\mathcal{F}_{1}\left(D\left(\bar{v}_{(\tilde{u}, \tilde{\theta})}+v^{0} \xi\right)\right)\right)\right. \\
& \left.: D\left(\bar{v}_{\left(\tilde{u}_{n}, \tilde{\theta}_{n}\right)}-\bar{v}_{(\tilde{u}, \tilde{\theta})}\right) d x d t\right)^{\frac{p}{2}} \\
& \times\left(2\left(C^{\text {flow }}+\left\|v^{0} \xi\right\|_{L^{p}\left(0, T ; V_{\Gamma_{1}}^{p}\right)}\right)^{p}\right)^{\frac{2-p}{2}},
\end{aligned}
$$

and finally,

$$
\begin{aligned}
& \frac{\mu_{0}(p-1)}{2^{(2-p)}\left(C^{\text {flow }}+\left\|v^{0} \xi\right\|_{L^{p}\left(0, T ; V_{\Gamma_{1}}^{p}\right)}\right)^{(2-p)}}\left\|D\left(\bar{v}_{\left(\tilde{u}_{n}, \tilde{\theta}_{n}\right)}-\bar{v}_{(\tilde{u}, \tilde{\theta})}\right)\right\|_{L^{p}\left(0, T ;\left(L^{p}(\Omega)\right)^{3 \times 3}\right)}^{2} \\
& \leq \int_{0}^{T} \int_{\Omega}\left(\mathcal{F}_{1}\left(D\left(\bar{v}_{\left(\tilde{u}_{n}, \tilde{\theta}_{n}\right)}+v^{0} \xi\right)\right)-\mathcal{F}_{1}\left(D\left(\bar{v}_{(\tilde{u}, \tilde{\theta})}+v^{0} \xi\right)\right)\right) \\
& \quad: D\left(\bar{v}_{\left(\tilde{u}_{n}, \tilde{\theta}_{n}\right)}-\bar{v}_{\left(\tilde{u}_{n}, \tilde{\theta}_{n}\right)}\right) d x d t
\end{aligned}
$$

Going back to (3.21), we obtain

$$
\begin{aligned}
& \frac{\mu_{0}(p-1)}{2^{(2-p)}\left(C^{\text {flow }}+\left\|v^{0} \xi\right\|_{L^{p}\left(0, T ; V_{\Gamma_{1}}^{p}\right)}\right)^{(2-p)}}\left\|D\left(\bar{v}_{\left(\tilde{u}_{n}, \tilde{\theta}_{n}\right)}-\bar{v}_{(\tilde{u}, \tilde{\theta})}\right)\right\|_{L^{p}\left(0, T ;\left(L^{p}(\Omega)\right)^{3 \times 3}\right)}^{2} \\
& \leq \int_{0}^{T} \int_{\Omega}\left(\mathcal{F}_{2}\left(\tilde{\theta}, \tilde{u}+v^{0} \xi, D\left(\bar{v}_{(\tilde{u}, \tilde{\theta})}+v^{0} \xi\right)\right)\right. \\
& \left.\quad-\mathcal{F}_{2}\left(\tilde{\theta}_{n}, \tilde{u}_{n}+v^{0} \xi, D\left(\bar{v}_{(\tilde{u}, \tilde{\theta})}+v^{0} \xi\right)\right)\right): D\left(\bar{v}_{\left(\tilde{u}_{n}, \tilde{\theta}_{n}\right)}-\bar{v}_{(\tilde{u}, \tilde{\theta})}\right) d x d t \\
& \leq \| \mathcal{F}_{2}\left(\tilde{\theta}, \tilde{u}+v^{0} \xi, D\left(\bar{v}_{(\tilde{u}, \tilde{\theta})}+v^{0} \xi\right)\right) \\
& \quad-\mathcal{F}_{2}\left(\tilde{\theta}_{n}, \tilde{u}_{n}+v^{0} \xi, D\left(\bar{v}_{(\tilde{u}, \tilde{\theta})}+v^{0} \xi\right)\right) \|_{L^{p^{\prime}}\left(0, T ;\left(L^{p^{\prime}}(\Omega)\right)^{3 \times 3}\right)} \\
& \quad \times\left\|D\left(\bar{v}_{\left(\tilde{u}_{n}, \tilde{\theta}_{n}\right)}-\bar{v}_{(\tilde{u}, \tilde{\theta})}\right)\right\|_{L^{p}\left(0, T ;\left(L^{p}(\Omega)\right)^{3 \times 3}\right)^{\prime}}
\end{aligned}
$$

which yields

$$
\begin{aligned}
& \frac{\mu_{0}(p-1)}{2^{(2-p)}\left(C^{\text {flow }}+\left\|v^{0} \xi\right\|_{L^{p}\left(0, T ; V_{\Gamma_{1}}^{p}\right)}\right)^{(2-p)}}\left\|D\left(\bar{v}_{\left(\tilde{u}_{n}, \tilde{\theta}_{n}\right)}-\bar{v}_{(\tilde{u}, \tilde{\theta})}\right)\right\|_{L^{p}\left(0, T ;\left(L^{p}(\Omega)\right)^{3 \times 3}\right)} \\
& \leq \| \mathcal{F}_{2}\left(\tilde{\theta}, \tilde{u}+v^{0} \xi, D\left(\bar{v}_{(\tilde{u}, \tilde{\theta})}+v^{0} \xi\right)\right) \\
& -\mathcal{F}_{2}\left(\tilde{\theta}_{n}, \tilde{u}_{n}+v^{0} \xi, D\left(\bar{v}_{(\tilde{u}, \tilde{\theta})}+v^{0} \xi\right)\right) \|_{L^{p^{\prime}}\left(0, T ;\left(L^{p^{\prime}}(\Omega)\right)^{3 \times 3}\right)^{3}}
\end{aligned}
$$

Case 2: $p \in[2,+\infty)$

Once again, with some algebraic computations, we get

$$
\left(\mathcal{F}_{1}(\lambda)-\mathcal{F}_{1}\left(\lambda^{\prime}\right)\right):\left(\lambda-\lambda^{\prime}\right) \geq \frac{\mu_{0}}{2^{p-1}}\left\|\lambda-\lambda^{\prime}\right\|^{p}
$$


for all $\left(\lambda, \lambda^{\prime}\right) \in \mathbb{R}^{3 \times 3} \times \mathbb{R}^{3 \times 3}$ (see, for instance, Theorem 4.1 in [11]). Going back to (3.21),

$$
\begin{aligned}
\frac{\mu_{0}}{2^{p-1}} & \left\|D\left(\bar{v}_{\left(\tilde{u}_{n}, \tilde{\theta}_{n}\right)}-\bar{v}_{(\tilde{u}, \tilde{\theta})}\right)\right\|_{L^{p}\left(0, T ;\left(L^{p}(\Omega)\right)^{3 \times 3}\right)}^{p} \\
\leq & \int_{0}^{T} \int_{\Omega}\left(\mathcal{F}_{2}\left(\tilde{\theta}, \tilde{u}+v^{0} \xi, D\left(\bar{v}_{(\tilde{u}, \tilde{\theta})}+v^{0} \xi\right)\right)-\mathcal{F}_{2}\left(\tilde{\theta}_{n}, \tilde{u}_{n}+v^{0} \xi, D\left(\bar{v}_{(\tilde{u}, \tilde{\theta})}+v^{0} \xi\right)\right)\right) \\
\quad & : D\left(\bar{v}_{\left(\tilde{u}_{n}, \tilde{\theta}_{n}\right)}-\bar{v}_{(\tilde{u}, \tilde{\theta})}\right) d x d t \\
\leq & \| \mathcal{F}_{2}\left(\tilde{\theta}, \tilde{u}+v^{0} \xi, D\left(\bar{v}_{(\tilde{u}, \tilde{\theta})}+v^{0} \xi\right)\right) \\
& -\mathcal{F}_{2}\left(\tilde{\theta}_{n}, \tilde{u}_{n}+v^{0} \xi, D\left(\bar{v}_{(\tilde{u}, \tilde{\theta})}+v^{0} \xi\right)\right) \|_{L^{p^{\prime}}\left(0, T ;\left(L^{p^{\prime}}(\Omega)\right)^{3 \times 3}\right)} \\
& \times \| D\left(\bar{v}_{\left(\tilde{u}_{n}, \tilde{\theta}_{n}\right)}-\bar{v}_{(\tilde{u}, \tilde{\theta})} \|_{L^{p}\left(0, T ;\left(L^{p}(\Omega)\right)^{3 \times 3}\right)^{*}} .\right.
\end{aligned}
$$

Thus,

$$
\begin{aligned}
\frac{\mu_{0}}{2^{p-1}} & \left\|D\left(\bar{v}_{\left(\tilde{u}_{n}, \tilde{\theta}_{n}\right)}-\bar{v}_{(\tilde{u}, \tilde{\theta})}\right)\right\|_{L^{p}\left(0, T ;\left(L^{p}(\Omega)\right)^{3 \times 3}\right)}^{p-1} \\
\leq & \| \mathcal{F}_{2}\left(\tilde{\theta}, \tilde{u}+v^{0} \xi, D\left(\bar{v}_{(\tilde{u}, \tilde{\theta})}+v^{0} \xi\right)\right) \\
& \quad-\mathcal{F}_{2}\left(\tilde{\theta}_{n}, \tilde{u}_{n}+v^{0} \xi, D\left(\bar{v}_{(\tilde{u}, \tilde{\theta})}+v^{0} \xi\right)\right) \|_{L^{p^{\prime}}\left(0, T ;\left(L^{p^{\prime}}(\Omega)\right)^{3 \times 3}\right)^{3}}
\end{aligned}
$$

By possibly extracting a subsequence still denoted $\left(\tilde{u}_{n}, \tilde{\theta}_{n}\right)_{n \geq 1}$, we have

$$
\tilde{u}_{n} \longrightarrow \tilde{u}, \quad \tilde{\theta}_{n} \longrightarrow \tilde{\theta} \quad \text { a.e. in }(0, T) \times \Omega .
$$

By using the continuity and boundedness assumptions (2.8) and (2.10) for the mapping $\mu$, we infer with Lebesgue's theorem that

$$
\begin{aligned}
& \mathcal{F}_{2}\left(\tilde{\theta}_{n}, \tilde{u}_{n}+v^{0} \xi, D\left(\bar{v}_{(\tilde{u}, \tilde{\theta})}+v^{0} \xi\right)\right) \longrightarrow \mathcal{F}_{2}\left(\tilde{\theta}, \tilde{u}+v^{0} \xi, D\left(\bar{v}_{(\tilde{u}, \tilde{\theta})}+v^{0} \xi\right)\right) \\
& \quad \text { strongly in } L^{p^{\prime}}\left(0, T ;\left(L^{p^{\prime}}(\Omega)\right)^{3 \times 3}\right) .
\end{aligned}
$$

With (3.22) when $p \in[6 / 5,2)$ or $(3.23)$ when $p \in[2,+\infty)$, we obtain

$$
\left\|D\left(\bar{v}_{\left(\tilde{u}_{n}, \tilde{\theta}_{n}\right)}-\bar{v}_{(\tilde{u}, \tilde{\theta})}\right)\right\|_{L^{p}\left(0, T ;\left(L^{p}(\Omega)\right)^{3 \times 3)}\right.} \longrightarrow 0
$$

and with Korn's inequality, we infer that

$$
\bar{v}_{\left(\tilde{u}_{n}, \tilde{\theta}_{n}\right)} \longrightarrow \bar{v}_{(\tilde{u}, \tilde{\theta})} \quad \text { strongly in } L^{p}\left(0, T ; V_{0 . \text { div }}^{p}\right) \text { and in } L^{p}\left(0, T ; \mathbf{L}^{p}(\Omega)\right)
$$

By possibly extracting another subsequence still denoted $\left(\bar{v}_{\left(\tilde{u}_{n}, \tilde{\theta}_{n}\right)}\right)_{n \geq 1}$, we have

$$
D\left(\bar{v}_{\left(\tilde{u}_{n}, \tilde{\theta}_{n}\right)}\right) \longrightarrow D\left(\bar{v}_{(\tilde{u}, \tilde{\theta})}\right), \quad \bar{v}_{\left(\tilde{u}_{n}, \tilde{\theta}_{n}\right)} \longrightarrow \bar{v}_{(\tilde{u}, \tilde{\theta})} \quad \text { a.e. in }(0, T) \times \Omega
$$

By using the continuity and boundedness properties of the mappings $\mu$ and $r$, we obtain

$$
g_{\delta}\left(\tilde{\theta}_{n}, \bar{v}_{\left(\tilde{u}_{n}, \tilde{\theta}_{n}\right)}\right) \longrightarrow g_{\delta}\left(\tilde{\theta}, \bar{v}_{(\tilde{u}, \tilde{\theta})}\right) \quad \text { strongly in } L^{2}\left(0, T ; L^{2}(\Omega)\right)
$$


and

$$
r\left(\tilde{\theta}_{n}\right) \longrightarrow r(\tilde{\theta}) \quad \text { strongly in } L^{2}\left(0, T ; L^{2}(\Omega)\right)
$$

Moreover, $\left(\theta_{\delta,\left(\bar{v}_{\left(\tilde{u}_{n}, \tilde{\theta}_{n}\right)}, \tilde{\theta}_{n}\right)}\right)_{n \geq 1}$ satisfies the estimate (3.18). It follows that there exists $\theta_{\delta}^{*} \in$ $W^{1,2}((0, T) \times \Omega) \cap L^{2}\left(0, T ; W_{\Gamma_{1} \cup \Gamma_{L}}^{1,2}(\Omega)\right)$ such that, by possibly extracting a subsequence still denoted $\left(\theta_{\delta,\left(\bar{v}_{\left(\tilde{u}_{n}, \tilde{\theta}_{n}\right)}, \tilde{\theta}_{n}\right)}\right)_{n \geq 1}$, we have

$$
\begin{aligned}
& \theta_{\delta,\left(\bar{v}_{\left(\tilde{u}_{n}, \tilde{\theta}_{n}\right)}, \tilde{\theta}_{n}\right)} \longrightarrow \theta_{\delta}^{*} \quad \text { weakly in } W^{1,2}((0, T) \times \Omega) \\
& \text { and weakly* in } L^{\infty}\left(0, T ; W_{\Gamma_{1} \cup \Gamma_{L}}^{1,2}(\Omega)\right)
\end{aligned}
$$

and with Simon's lemma [34]

$$
\theta_{\delta,\left(\bar{v}_{\delta,\left(\tilde{u}_{n}, \tilde{\theta}_{n}\right)}, \tilde{\theta}_{n}\right)} \longrightarrow \theta_{\delta}^{*} \quad \text { strongly in } C^{0}\left([0, T] ; L^{2}(\Omega)\right) \text { and in } L^{2}\left(0, T ; L^{2}(\Omega)\right) \text {. }
$$

Hence, we may pass to the limit in all the terms of $\left(P_{\delta,\left(\bar{v}_{\left(\tilde{u}_{n}, \tilde{\theta}_{n}\right)}^{\text {heat }}, \tilde{\theta}_{n}\right)}\right)$ and obtain that $\theta_{\delta}^{*}$ is

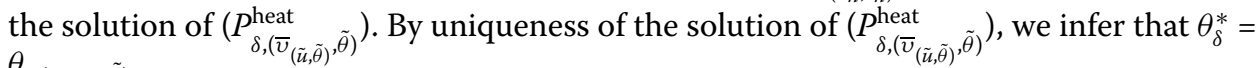
$\theta_{\delta,(\bar{v}}(\tilde{u}, \tilde{\theta})^{, \tilde{\theta}} \cdot{ }^{*}$

Finally, recalling that $\left(\bar{v}_{\left(\tilde{u}_{n}, \tilde{\theta}_{n}\right)}, \theta_{\delta,\left(\bar{v}_{\left(\tilde{u}_{n}, \tilde{\theta}_{n}\right)}, \tilde{\theta}_{n}\right)}\right)_{n \geq 1}$ satisfies the estimates (3.11)-(3.13) and (3.18), we infer that the whole sequence $\left(\bar{v}_{\left(\tilde{u}_{n}, \tilde{\theta}_{n}\right)}, \theta_{\delta,\left(\bar{v}_{\left(\tilde{u}_{n}, \tilde{\theta}_{n}\right)}, \tilde{\theta}_{n}\right)}\right)_{n \geq 1}$ converges strongly to $\left(\bar{v}_{(\tilde{u}, \tilde{\theta})}, \theta_{\delta,\left(\bar{v}_{(\tilde{u}, \tilde{\theta})}, \tilde{\theta}\right)}\right)$ in the space $L^{p}\left(0, T ; \mathbf{L}^{p}(\Omega)\right) \times L^{2}\left(0, T ; L^{2}(\Omega)\right)$, which allows us to conclude.

As a corollary, we obtain an existence result for the approximate problem $\left(P_{\delta}\right)$.

Corollary 3.6 Let $\delta>0$. Let the mappings $c, K, r$, and $\mu$ satisfy (2.3)-(2.10), $f \in$ $L^{p^{\prime}}\left(0, T ; \mathbf{L}^{2}(\Omega)\right), k \in L^{p^{\prime}}\left(0, T ; L_{+}^{p^{\prime}}\left(\Gamma_{0}\right)\right), s \in L^{p}\left(0, T ; \mathbf{L}^{p}\left(\Gamma_{0}\right)\right), \xi \in W^{1, p^{\prime}}(0, T)$ satisfying (1.6), $\theta_{\delta}^{0} \in \mathcal{D}(\Omega), \theta_{\delta}^{b} \in \mathcal{D}((0, T) \times \omega)$ and $v^{0} \in \mathbf{W}^{1, p}(\Omega)$ satisfying $(2.12)$. Then problem $\left(P_{\delta}\right)$ admits a solution.

Proof With the previous theorem, we know that the mapping $T_{\delta}$ admits a fixed point, i.e., there exists $(\tilde{u}, \tilde{\theta}) \in L^{p}\left(0, T ; \mathbf{L}^{p}(\Omega)\right) \times L^{2}\left(0, T ; L^{2}(\Omega)\right)$ such that

$$
T_{\delta}(\tilde{u}, \tilde{\theta})=\left(\bar{v}_{(\tilde{u}, \tilde{\theta})}, \theta_{\delta,(\bar{v}}(\tilde{u}, \tilde{\theta}, \tilde{\theta})=(\tilde{u}, \tilde{\theta})\right.
$$

By the definition of $T_{\delta}$, we infer that $\tilde{u}=\bar{v}_{(\tilde{u}, \tilde{\theta})} \in C\left([0, T] ; \mathbf{L}^{2}(\Omega)\right) \cap L^{p}\left(0, T ; V_{0 \text { div }}^{p}\right)$ with $\frac{\partial \tilde{u}}{\partial t}=$ $\frac{\partial \bar{v}_{(\tilde{u}, \tilde{\theta})}}{\partial t} \in L^{p^{\prime}}\left(0, T ;\left(V_{0 . \operatorname{div}}^{p}\right)^{\prime}\right)$, and there exists $\pi_{(\tilde{u}, \tilde{\theta})} \in H^{-1}\left(0, T ; L_{0}^{p^{\prime}}(\Omega)\right)$ such that $\left(\tilde{u}, \pi_{(\tilde{u}, \tilde{\theta})}\right)=$

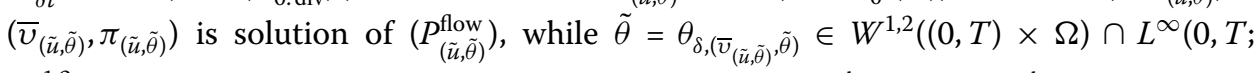

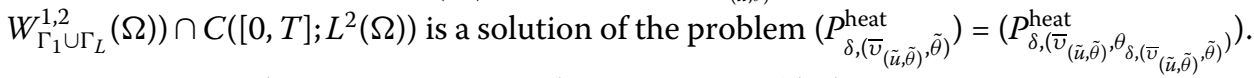
Hence, the triplet $\left.\left(\bar{v}_{(\tilde{u}, \tilde{\theta})}, \pi_{(\tilde{u}, \tilde{\theta})}, \theta_{\delta,(\bar{v}(\tilde{u}, \tilde{\theta})}, \tilde{\theta}\right)\right)$ is a solution of $\left(P_{\delta}\right)$. Aubin and Simon

\section{Existence result for the coupled fluid flow / heat transfer problem $(P)$}

In order to prove the existence of a solution to the coupled fluid flow/heat transfer problem $(P)$, we consider a sequence of approximate solutions and prove its convergence to a triplet $(\bar{v}, \pi, \theta)$, satisfying $(2.13)-(2.15)$. 
More precisely, let $\delta_{n}=\frac{1}{n}$ for all $n \geq 1$ and $\left(\bar{v}_{n}, \pi_{n}, \theta_{n}\right)$ be a solution of the approximate problem $\left(P_{\delta_{n}}\right)$. For all $n \geq 1$, we have $\theta_{n} \in W^{1,2}((0, T) \times \Omega) \cap L^{\infty}\left(0, T ; W_{\Gamma_{1} \cup \Gamma_{L}}^{1,2}(\Omega)\right) \cap$ $C^{0}\left([0, T] ; L^{2}(\Omega)\right), \bar{v}_{n} \in C\left([0, T] ; \mathbf{L}^{2}(\Omega)\right) \cap L^{p}\left(0, T ; V_{0 . \text { div }}^{p}\right)$ with $\frac{\partial \bar{v}_{n}}{\partial t} \in L^{p^{\prime}}\left(0, T ;\left(V_{0 . \text { div }}^{p}\right)^{\prime}\right)$ and $\pi_{n} \in H^{-1}\left(0, T ; L_{0}^{p^{\prime}}(\Omega)\right)$ such that

$$
\begin{aligned}
\left\langle\frac{\partial}{\partial t}\left(\bar{v}_{n}, \tilde{\vartheta}\right)_{\mathbf{L}^{2}(\Omega)}, \zeta\right\rangle_{\mathcal{D}^{\prime}(0, T), \mathcal{D}(0, T)} \\
\quad+\int_{0}^{T} \int_{\Omega} \mathcal{F}\left(\theta_{n}, \bar{v}_{n}+v^{0} \xi, D\left(\bar{v}_{n}+v^{0} \xi\right)\right): D(\tilde{\vartheta}) \zeta d x d t \\
\quad-\left\langle\int_{\Omega} \pi_{n} \operatorname{div}(\tilde{\vartheta}) d x, \zeta\right\rangle_{\mathcal{D}^{\prime}(0, T), \mathcal{D}(0, T)}+J\left(\bar{v}_{n}+\tilde{\vartheta} \zeta\right)-J\left(\bar{v}_{n}\right) \\
\geq \int_{0}^{T}\left(f+v^{0} \frac{\partial \xi}{\partial t}, \tilde{\vartheta}\right)_{\mathbf{L}^{2}(\Omega)} \zeta d t \quad \forall \tilde{\vartheta} \in V_{0}^{p}, \forall \zeta \in \mathcal{D}(0, T)
\end{aligned}
$$

and

$$
\begin{aligned}
\int_{0}^{T} & \int_{\Omega} c \frac{\partial \theta_{n}}{\partial t} w \widetilde{\zeta} d x d t+\int_{0}^{T} \int_{\Omega}\left(K \nabla \theta_{n}\right) \cdot \nabla w \widetilde{\zeta} d x d t \\
= & \int_{0}^{T} \int_{\Omega} g_{\delta_{n}}\left(\theta_{n}, \bar{v}_{n}\right) w \widetilde{\zeta} d x d t+\int_{0}^{T} \int_{\Omega} r\left(\theta_{n}\right) w \widetilde{\zeta} d x d t \\
& \quad+\int_{0}^{T} \int_{\omega} \theta_{\delta_{n}}^{b} w \widetilde{\zeta} d x^{\prime} d t \quad \forall w \in W_{\Gamma_{1} \cup \Gamma_{L}}^{1,2}(\Omega), \forall \widetilde{\zeta} \in \mathcal{D}(0, T)
\end{aligned}
$$

with the initial conditions

$$
\bar{v}_{n}(0, \cdot)=0 \quad \text { in } \Omega
$$

and

$$
\theta_{n}(0, \cdot)=\theta_{\delta_{n}}^{0} \quad \text { in } \Omega
$$

Owing to that $\theta_{n} \in W^{1,2}((0, T) \times \Omega) \cap L^{\infty}\left(0, T ; W_{\Gamma_{1} \cup \Gamma_{L}}^{1,2}(\Omega)\right) \cap C^{0}\left([0, T] ; L^{2}(\Omega)\right)$ the variational equality (4.2) remains true for all $\widetilde{\zeta} \in L^{2}(0, T ; \mathbb{R})$. Let us choose $\widetilde{\zeta} \in C^{\infty}([0, T])$ such that $\tilde{\zeta}(T)=0$ : with integration by part with respect to the time-variable, we obtain

$$
\begin{aligned}
&- \int_{0}^{T} \int_{\Omega} c \theta_{n} w \widetilde{\zeta}^{\prime} d x d t+\int_{0}^{T} \int_{\Omega}\left(K \nabla \theta_{n}\right) \cdot \nabla w \tilde{\zeta} d x d t \\
&= \int_{0}^{T} \int_{\Omega} g_{\delta_{n}}\left(\theta_{n}, \bar{v}_{n}\right) w \widetilde{\zeta} d x d t+\int_{0}^{T} \int_{\Omega} r\left(\theta_{n}\right) w \widetilde{\zeta} d x d t \\
& \quad+\int_{0}^{T} \int_{\omega} \theta_{\delta_{n}}^{b} w \widetilde{\zeta} d x^{\prime} d t+\int_{\Omega} c \theta_{\delta_{n}}^{0} w \widetilde{\zeta}(0) d x \\
& \forall w \in W_{\Gamma_{1} \cup \Gamma_{L}}^{1,2}(\Omega), \quad \forall \widetilde{\zeta} \in C^{\infty}([0, T]) \text { s.t. } \widetilde{\zeta}(T)=0 .
\end{aligned}
$$

Let us establish first some a priori estimates for $\left(\bar{v}_{n}, \pi_{n}\right)_{n \geq 1}$. 
Proposition 4.1 (A priori estimates of $\left.\left(\bar{v}_{n}, \pi_{n}\right)_{n \geq 1}\right)$ Let $\mu$ satisfy (2.8)-(2.10), $f \in L^{p^{\prime}}(0, T$; $\left.\mathbf{L}^{2}(\Omega)\right), k \in L^{p^{\prime}}\left(0, T ; L_{+}^{p^{\prime}}\left(\Gamma_{0}\right)\right), s \in L^{p}\left(0, T ; \mathbf{L}^{p}\left(\Gamma_{0}\right)\right), \xi \in W^{1, p^{\prime}}(0, T)$ satisfying $(1.6)$, and $v^{0} \in$ $\mathbf{W}^{1, p}(\Omega)$ satisfying (2.12). Then there exists a constant $\widetilde{C}^{\text {flow }}$, independent of $n$, such that for all $n \geq 1$ :

$$
\begin{aligned}
& \left\|\bar{v}_{n}\right\|_{L^{p}\left(0, T ; V_{0 . \text { div }}^{p}\right)} \leq \widetilde{C}^{\text {flow }}, \\
& \left\|\bar{v}_{n}\right\|_{L^{\infty}\left(0, T ; \mathbf{L}^{2}(\Omega)\right)} \leq \widetilde{C}^{\text {flow }}, \\
& \left\|\frac{\partial \bar{v}_{n}}{\partial t}\right\|_{L^{p^{\prime}}\left(0, T ;\left(V_{0 . \text { div }}^{p}\right)^{\prime}\right)} \leq \widetilde{C}^{\text {flow }}
\end{aligned}
$$

and

$$
\left\|\pi_{n}\right\|_{H^{-1}\left(0, T ; L_{0}^{p^{\prime}}(\Omega)\right)} \leq \widetilde{C}^{\text {flow }}
$$

Proof By using the same computations as in Proposition 3.2, we obtain immediately (4.6)(4.8).

Now let us prove (4.9). We choose $\bar{\varphi}= \pm \tilde{\vartheta} \zeta$ with $\tilde{\vartheta} \in \mathbf{W}_{0}^{1, p}(\Omega)$ and $\zeta \in \mathcal{D}(0, T)$ in (4.1). We obtain

$$
\begin{aligned}
\left\langle\int_{\Omega}\right. & \left.\pi_{n} \operatorname{div}(\tilde{\vartheta}) d x, \zeta\right\rangle_{\mathcal{D}^{\prime}(0, T), \mathcal{D}(0, T)} \\
= & -\int_{0}^{T}\left(\bar{v}_{n}, \tilde{\vartheta}\right)_{\mathbf{L}^{2}(\Omega)} \zeta^{\prime} d t \\
& +\int_{0}^{T} \int_{\Omega} \mathcal{F}\left(\theta_{n}, \bar{v}_{n}+v^{0} \xi, D\left(\bar{v}_{n}+v^{0} \xi\right)\right): D(\tilde{\vartheta}) \zeta d x d t \\
& -\int_{0}^{T}\left(f+v^{0} \frac{\partial \xi}{\partial t}, \tilde{\vartheta}\right)_{\mathbf{L}^{2}(\Omega)} \zeta d t .
\end{aligned}
$$

Thus,

$$
\begin{aligned}
& \left|\left\langle\int_{\Omega} \pi_{n} \operatorname{div}(\tilde{\vartheta}) d x, \zeta\right\rangle_{\mathcal{D}^{\prime}(0, T), \mathcal{D}(0, T)}\right| \\
& \leq \quad \sqrt{T}\left\|\bar{v}_{n}\right\|_{L^{\infty}\left(0, T ; \mathbf{L}^{2}(\Omega)\right)}\left\|\tilde{\vartheta} \zeta^{\prime}\right\|_{L^{2}\left(0, T ; \mathbf{L}^{2}(\Omega)\right)} \\
& \quad+2 \mu_{1}\left(\left\|\bar{v}_{n}\right\|_{L^{p}\left(0, T ; V_{0, \text { div }}^{p}\right)}+\left\|v^{0} \xi\right\|_{L^{p}\left(0, T ; V_{\Gamma_{1}}^{p}\right)}\right)^{p-1}\|\tilde{\vartheta} \zeta\|_{L^{p}\left(0, T ; V_{0}^{p}\right)} \\
& \quad+\left\|f+v^{0} \frac{\partial \xi}{\partial t}\right\|_{L^{p^{\prime}\left(0, T ; \mathbf{L}^{2}(\Omega)\right)}}\|\tilde{\vartheta} \zeta\|_{L^{p}\left(0, T ; \mathbf{L}^{2}(\Omega)\right)}
\end{aligned}
$$

and with (4.6)-(4.8), we get

$$
\begin{aligned}
& \left|\left\langle\int_{\Omega} \pi_{n} \operatorname{div}(\tilde{\vartheta}) d x, \zeta\right\rangle_{\mathcal{D}^{\prime}(0, T), \mathcal{D}(0, T)}\right| \\
& \leq\left(\sqrt{T} \widetilde{C} \widetilde{C}^{\text {flow }}+2 \mu_{1} C_{\infty} T^{1 / p}\left(\widetilde{C}^{\text {flow }}+\left\|v^{0} \xi\right\|_{L^{p}\left(0, T ; V_{\Gamma_{1}}^{p}\right)}\right)^{p-1}\right. \\
& \left.\quad+\widetilde{C} C_{\infty} T^{1 / p}\left\|f+v^{0} \frac{\partial \xi}{\partial t}\right\|_{L^{p^{\prime}}\left(0, T ; \mathbf{L}^{2}(\Omega)\right)}\right)\|\tilde{\vartheta} \zeta\|_{H_{0}^{1}\left(0, T ; V_{0}^{p}\right)},
\end{aligned}
$$


where $\widetilde{C}$ denotes the norm of the continuous injection of $V_{0}^{p}$ into $\mathbf{L}^{2}(\Omega)$, and $C_{\infty}$ is the norm of the continuous injection of $H^{1}(0, T ; \mathbb{R})$ into $L^{\infty}(0, T ; \mathbb{R})$.

Moreover, for any $p>1$, there exists a linear and continuous operator $P_{p}: L_{0}^{p}(\Omega) \rightarrow$ $\mathbf{W}_{0}^{1, p}(\Omega)$ such that

$$
\operatorname{div}\left(P_{p}(\varpi)\right)=\varpi \quad \forall \varpi \in L_{0}^{p}(\Omega)
$$

(see Corollary 3.1 in [2]). It follows that for any $\varpi \in L_{0}^{p}(\Omega)$ and $\zeta \in \mathcal{D}(0, T)$, we have

$$
\begin{aligned}
& \left|\left\langle\int_{\Omega} \pi_{n} \varpi d x, \zeta\right\rangle_{\mathcal{D}^{\prime}(0, T), \mathcal{D}(0, T)}\right| \\
& \leq\left\|P_{p}\right\|_{\mathcal{L}\left(L_{0}^{p}(\Omega), \mathbf{w}_{0}^{1, p}(\Omega)\right)}\left(\sqrt{T} \widetilde{C} \widetilde{C}^{\text {flow }}+2 \mu_{1} C_{\infty} T^{1 / p}\left(\widetilde{C}^{\text {flow }}+\left\|v^{0} \xi\right\|_{L^{p}\left(0, T ; V_{\Gamma_{1}}^{p}\right)}\right)^{p-1}\right. \\
& \left.\quad+\widetilde{C} C_{\infty} T^{1 / p}\left\|f+v^{0} \frac{\partial \xi}{\partial t}\right\|_{L^{p^{\prime}\left(0, T ; \mathbf{L}^{2}(\Omega)\right)}}\right)\|\varpi \zeta\|_{H_{0}^{1}\left(0, T ; L^{p}(\Omega)\right)} .
\end{aligned}
$$

Hence, there exists a real number $C^{\prime}>0$, independent of $n$, such that for all $n \geq 1$, we have

$$
\left|\left\langle\int_{\Omega} \pi_{n} \varpi d x, \zeta\right\rangle_{\mathcal{D}^{\prime}(0, T), \mathcal{D}(0, T)}\right| \leq C^{\prime}\|\varpi \zeta\|_{H_{0}^{1}\left(0, T ; L^{p}(\Omega)\right)} \quad \forall \varpi \in L_{0}^{p}(\Omega), \forall \zeta \in \mathcal{D}(0, T) .
$$

Furthermore, for any $\varpi^{*} \in L^{p}(\Omega)$, we may define $\varpi \in L_{0}^{p}(\Omega)$ by

$$
\varpi=\varpi^{*}-\frac{1}{\operatorname{meas}(\Omega)} \int_{\Omega} \varpi^{*} d x
$$

We have $\|\varpi\|_{L^{p}(\Omega)} \leq 2\left\|\varpi^{*}\right\|_{L^{p}(\Omega)}$, and since $\pi_{n} \in H^{-1}\left(0, T ; L_{0}^{p^{\prime}}(\Omega)\right)$, we have

$$
\begin{aligned}
\left\langle\int_{\Omega} \pi_{n}\left(\varpi^{*}-\frac{1}{\operatorname{meas}(\Omega)} \int_{\Omega} \varpi^{*} d x\right) d x, \zeta\right\rangle_{\mathcal{D}^{\prime}(0, T), \mathcal{D}(0, T)} \\
=\left\langle\int_{\Omega} \pi_{n} \varpi^{*} d x, \zeta\right\rangle_{\mathcal{D}^{\prime}(0, T), \mathcal{D}(0, T)} \\
\left.\quad-\frac{1}{\operatorname{meas}(\Omega)}\left(\int_{\Omega} \varpi^{*} d x\right)\right)\left\langle\int_{\Omega} \pi_{n} d x, \zeta\right\rangle_{\mathcal{D}^{\prime}(0, T), \mathcal{D}(0, T)} \\
=\left\langle\int_{\Omega} \pi_{n} \varpi^{*} d x, \zeta\right\rangle_{\mathcal{D}^{\prime}(0, T), \mathcal{D}(0, T)} .
\end{aligned}
$$

It follows that

$$
\begin{aligned}
& \left|\left\langle\int_{\Omega} \pi_{n} \varpi^{*} d x, \zeta\right\rangle_{\mathcal{D}^{\prime}(0, T), \mathcal{D}(0, T)}\right| \\
& \quad=\left|\left\langle\int_{\Omega} \pi_{n} \varpi d x, \zeta\right\rangle_{\mathcal{D}^{\prime}(0, T), \mathcal{D}(0, T)}\right| \\
& \quad \leq 2 C^{\prime}\left\|\varpi^{*} \zeta\right\|_{H_{0}^{1}\left(0, T ; L^{p}(\Omega)\right)} \quad \forall \varpi^{*} \in L^{p}(\Omega), \forall \zeta \in \mathcal{D}(0, T) .
\end{aligned}
$$


Hence, by possibly modifying $\widetilde{C}^{\text {flow }}$, we have

$$
\begin{aligned}
& \left|\left\langle\int_{\Omega} \pi_{n} \varpi^{*} d x, \zeta\right\rangle_{\mathcal{D}^{\prime}(0, T), \mathcal{D}(0, T)}\right| \leq \widetilde{C}^{\text {flow }}\left\|\varpi^{*} \zeta\right\|_{H_{0}^{1}\left(0, T ; L^{p}(\Omega)\right)} \\
& \forall \varpi^{*} \in L^{p}(\Omega), \forall \zeta \in \mathcal{D}(0, T)
\end{aligned}
$$

and we may conclude by using the density of $\mathcal{D}(0, T) \otimes L^{p}(\Omega)$ into $H_{0}^{1}\left(0, T ; L^{p}(\Omega)\right)$.

Let us now obtain some a priori estimates for $\left(\theta_{n}\right)_{n \geq 1}$.

Proposition 4.2 (Further a priori estimates for $\theta_{n}$ ) Let $q \in\left(1, \frac{5}{4}\right)$. Under the previous assumptions, there exists a constant $C_{q}^{\text {heat }}>0$, independent of $n$, such that

$$
\left\|\theta_{n}\right\|_{L^{q}\left(0, T ; W_{\Gamma_{1} \cup \Gamma_{L}}^{1, q}(\Omega)\right)} \leq C_{q}^{\text {heat }}
$$

for all $n \geq 1$.

Proof Let $\widetilde{w}=w \widetilde{\zeta}$ with $w \in W_{\Gamma_{1} \cup \Gamma_{L}}^{1,2}(\Omega)$ and $\zeta \in \mathcal{D}(0, T)$. With (4.2), we have

$$
\begin{aligned}
& \int_{0}^{T} \int_{\Omega} c \frac{\partial \theta_{n}}{\partial t} \widetilde{w} d x d t+\int_{0}^{T} \int_{\Omega}\left(K \nabla \theta_{n}\right) \cdot \nabla \widetilde{w} d x d t \\
& \quad=\int_{0}^{T} \int_{\Omega} g_{\delta_{n}}\left(\theta_{n}, \bar{v}_{n}\right) \widetilde{w} d x d t+\int_{0}^{T} \int_{\Omega} r\left(\theta_{n}\right) \widetilde{w} d x d t+\int_{0}^{T} \int_{\omega} \theta_{\delta_{n}}^{b} \widetilde{w} d x^{\prime} d t
\end{aligned}
$$

and by density of $\mathcal{D}(0, T) \otimes W_{\Gamma_{1} \cup \Gamma_{L}}^{1,2}(\Omega)$ into $L^{2}\left(0, T ; W_{\Gamma_{1} \cup \Gamma_{L}}^{1,2}(\Omega)\right)$ the same equality holds for all $\widetilde{w} \in L^{2}\left(0, T ; W_{\Gamma_{1} \cup \Gamma_{L}}^{1,2}(\Omega)\right)$.

In order to obtain (4.10), we perform the same kind of computations as in [6]. More precisely, for all $m \geq 1$ let $\Psi_{0}^{m}: \mathbb{R} \rightarrow \mathbb{R}$ and $\Psi_{0}: \mathbb{R} \rightarrow \mathbb{R}$ be given by

$$
\Psi_{0}^{m}(s)=\left\{\begin{array}{ll}
0 & \text { if } s \geq 1+\frac{1}{m} \\
1-m(s-1) & \text { if } 1<s<1+\frac{1}{m}, \\
1 & \text { if }-1 \leq s \leq 1, \\
1+m(s+1) & \text { if }-1-\frac{1}{m}<s<-1, \\
0 & \text { if } s \leq-1-\frac{1}{m}
\end{array} \quad \Psi_{0}(s)= \begin{cases}0 & \text { if } s>1, \\
1 & \text { if }-1 \leq s \leq 1 \\
0 & \text { if } s<-1\end{cases}\right.
$$

and let

$$
\begin{array}{ll}
\psi_{0}^{m}(s)=\int_{0}^{s} \Psi_{0}^{m}(z) d z, & \psi_{0}(s)=\int_{0}^{s} \Psi_{0}(z) d z \quad \text { for all } s \in \mathbb{R}, \\
\Phi_{0}^{m}(s)=\int_{0}^{s} \psi_{0}^{m}(z) d z, & \Phi_{0}(s)=\int_{0}^{s} \psi_{0}(z) d z \quad \text { for all } s \in \mathbb{R} .
\end{array}
$$


We choose $\widetilde{w}=\psi_{0}^{m}\left(\theta_{n}\right) \mathbf{1}_{[0, t]}$, where $\mathbf{1}_{[0, t]}$ is the indicatrix function of the time-interval $[0, t]$ with $t \in(0, T]$, in (4.11). We obtain

$$
\begin{aligned}
& \int_{\Omega} c(x) \Phi_{0}^{m}\left(\theta_{n}(t, x)\right) d x \\
& \quad+\int_{0}^{t} \int_{\Omega} \Psi_{0}^{m}\left(\theta_{n}(s, x)\right)\left(K(x) \nabla \theta_{n}(s, x)\right) \cdot \nabla \theta_{n}(s, x) d x d s \\
&=\int_{0}^{t} \int_{\Omega} g_{\delta_{n}}\left(\theta_{n}, \bar{v}_{n}\right) \psi_{0}^{m}\left(\theta_{n}\right) d x d s+\int_{0}^{t} \int_{\Omega} r\left(\theta_{n}\right) \psi_{0}^{m}\left(\theta_{n}\right) d x d s \\
& \quad+\int_{0}^{t} \int_{\omega} \theta_{\delta_{n}}^{b} \psi_{0}^{m}\left(\theta_{n}\right) d x d s+\int_{\Omega} c(x) \Phi_{0}^{m}\left(\theta_{\delta_{n}}^{0}\right) d x .
\end{aligned}
$$

By observing that $\left|\Psi_{0}^{m}(s)\right| \leq 1,\left|\psi_{0}^{m}(s)\right| \leq 2$ and $\left|\Phi_{0}^{m}(s)\right| \leq 2|s|$ for all $s \in \mathbb{R}$ and for all $m \geq 1$, we may pass to the limit as $m$ tends to $+\infty$ by using Lebesgue's theorem, and we get

$$
\begin{aligned}
\int_{\Omega} c(x) & \Phi_{0}\left(\theta_{n}(t, x)\right) d x \\
& +\int_{0}^{t} \int_{\Omega} \Psi_{0}\left(\theta_{n}(s, x)\right)\left(K(x) \nabla \theta_{n}(s, x)\right) \cdot \nabla \theta_{n}(s, x) d x d s \\
= & \int_{0}^{t} \int_{\Omega} g_{\delta_{n}}\left(\theta_{n}, \bar{v}_{n}\right) \psi_{0}\left(\theta_{n}\right) d x d s+\int_{0}^{t} \int_{\Omega} r\left(\theta_{n}\right) \psi_{0}\left(\theta_{n}\right) d x d s \\
& +\int_{0}^{t} \int_{\omega} \theta_{\delta_{n}}^{b} \psi_{0}\left(\theta_{n}\right) d x^{\prime} d s+\int_{\Omega} c(x) \Phi_{0}\left(\theta_{\delta_{n}}^{0}\right) d x .
\end{aligned}
$$

Furthermore, $|s|-\frac{1}{2} \leq \Phi_{0}(s) \leq|s|$ and $\left|\psi_{0}(s)\right| \leq 1$ for all $s \in \mathbb{R}$. Thus,

$$
\begin{aligned}
c_{0} \int_{\Omega}\left|\theta_{n}(t, x)\right| d x+k_{0} \int_{0}^{t} \int_{\Omega} \underbrace{\Psi_{0}\left(\theta_{n}(s, x)\right)\left\|\nabla \theta_{n}(s, x)\right\|^{2}}_{\geq 0} d x d s \\
\leq\left\|g_{\delta_{n}}\left(\theta_{n}, \bar{v}_{n}\right)\right\|_{L^{1}\left(0, T ; L^{1}(\Omega)\right)}+\left\|r\left(\theta_{n}\right)\right\|_{L^{1}\left(0, T ; L^{1}(\Omega)\right)}+\left\|\theta_{\delta_{n}}^{b}\right\|_{L^{1}((0, T) \times \omega)} \\
\quad+\frac{1}{2}\|c\|_{L^{1}(\Omega)}+\left\|c \theta_{\delta_{n}}^{0}\right\|_{L^{1}(\Omega)}
\end{aligned}
$$

for all $t \in[0, T]$. Next, for all $k \geq 1$ and for all $m \geq 1$, we define $\Psi_{k}^{m}: \mathbb{R} \rightarrow \mathbb{R}$ and $\Psi_{k}: \mathbb{R} \rightarrow \mathbb{R}$ by

$$
\Psi_{k}^{m}(s)= \begin{cases}0 & \text { if } s \geq k+1+\frac{1}{m}, \\ 1-m(s-(k+1)) & \text { if } k+1<s<k+1+\frac{1}{m}, \\ 1 & \text { if } k+\frac{1}{m} \leq s \leq k+1, \\ m(s-k) & \text { if } k<s<k+\frac{1}{m}, \\ 0 & \text { if }-k \leq s \leq k, \\ -m(s+k) & \text { if }-k-\frac{1}{m}<s<-k, \\ 1 & \text { if }-k-1 \leq s \leq-k-\frac{1}{m}, \\ 1+m(s-(-k-1)) & \text { if }-k-1-\frac{1}{m}<s<-k-1, \\ 0 & \text { if } s \leq-k-1-\frac{1}{m},\end{cases}
$$




$$
\Psi_{k}(s)= \begin{cases}0 & \text { if } s>k+1, \\ 1 & \text { if } k<s \leq k+1, \\ 0 & \text { if }-k \leq s \leq k, \\ 1 & \text { if }-k-1 \leq s<-k, \\ 0 & \text { if } s<-k-1\end{cases}
$$

and let

$$
\begin{array}{ll}
\psi_{k}^{m}(s)=\int_{0}^{s} \Psi_{k}^{m}(z) d z, & \psi_{k}(s)=\int_{0}^{s} \Psi_{k}(z) d z \quad \text { for all } s \in \mathbb{R}, \\
\Phi_{k}^{m}(s)=\int_{0}^{s} \psi_{k}^{m}(z) d z, & \Phi_{k}(s)=\int_{0}^{s} \psi_{k}(z) d z
\end{array}
$$

Now we choose $\widetilde{w}=\psi_{k}^{m}\left(\theta_{n}\right)$ in (4.11) with $k \geq 0$ and $m \geq 1$. We get

$$
\begin{aligned}
& \int_{\Omega} c(x) \Phi_{k}^{m}\left(\theta_{n}(t, x)\right) d x \\
&+\int_{0}^{t} \int_{\Omega} \Psi_{k}^{m}\left(\theta_{n}(s, x)\right)\left(K(x) \nabla \theta_{n}(s, x)\right) \cdot \nabla \theta_{n}(s, x) d x d s \\
&= \int_{0}^{t} \int_{\Omega} g_{\delta_{n}}\left(\theta_{n}, \bar{v}_{n}\right) \psi_{k}^{m}\left(\theta_{n}\right) d x d s+\int_{0}^{t} \int_{\Omega} r\left(\theta_{n}\right) \psi_{k}^{m}\left(\theta_{n}\right) d x d s \\
& \quad+\int_{0}^{t} \int_{\omega} \theta_{\delta_{n}}^{b} \psi_{k}^{m}\left(\theta_{n}\right) d x^{\prime} d s+\int_{\Omega} c(x) \Phi_{k}^{m}\left(\theta_{\delta_{n}}^{0}\right) d x .
\end{aligned}
$$

We may observe that $\left|\Psi_{k}^{m}(s)\right| \leq 1,\left|\psi_{k}^{m}(s)\right| \leq 2$, and $\left|\Phi_{k}^{m}(s)\right| \leq 2|s|$ for all $s \in \mathbb{R}$, for all $k \geq 0$ and for all $m \geq 1$. By passing to the limit as $m$ tends to $+\infty$, we obtain

$$
\begin{aligned}
\int_{\Omega} c(x) & \Phi_{k}\left(\theta_{n}(t, x)\right) d x \\
& +\int_{0}^{t} \int_{\Omega} \Psi_{k}\left(\theta_{n}(s, x)\right)\left(K(x) \nabla \theta_{n}(s, x)\right) \cdot \nabla \theta_{n}(s, x) d x d s \\
= & \int_{0}^{t} \int_{\Omega} g_{\delta_{n}}\left(\theta_{n}, \bar{v}_{n}\right) \psi_{k}\left(\theta_{n}\right) d x d s+\int_{0}^{t} \int_{\Omega} r\left(\theta_{n}\right) \psi_{k}\left(\theta_{n}\right) d x d s \\
& +\int_{0}^{t} \int_{\omega} \theta_{\delta_{n}}^{b} \psi_{k}\left(\theta_{n}\right) d x^{\prime} d s+\int_{\Omega} c(x) \Phi_{k}\left(\theta_{\delta_{n}}^{0}\right) d x .
\end{aligned}
$$

Obviously $0 \leq \Phi_{k}(s) \leq|s|$ and $\left|\psi_{k}(s)\right| \leq 1$ for all $s \in \mathbb{R}$ and for all $k \geq 0$. Hence,

$$
\begin{aligned}
& k_{0} \int_{0}^{T} \int_{\Omega} \Psi_{k}\left(\theta_{n}\right)\left\|\nabla \theta_{n}\right\|^{2} d x d t \\
& \leq\left\|g_{\delta_{n}}\left(\theta_{n}, \bar{v}_{n}\right)\right\|_{L^{1}\left(0, T ; L^{1}(\Omega)\right)} \\
& \quad+\left\|r\left(\theta_{n}\right)\right\|_{L^{1}\left(0, T ; L^{1}(\Omega)\right)}+\left\|\theta_{\delta_{n}}^{b}\right\|_{L^{1}((0, T) \times \omega)}+\left\|c \theta_{\delta_{n}}^{0}\right\|_{L^{1}(\Omega)} .
\end{aligned}
$$


Let us define $C_{\delta_{n}}$ as

$$
\begin{aligned}
C_{\delta_{n}}= & \left\|g_{\delta_{n}}\left(\theta_{n}, \bar{v}_{n}\right)\right\|_{L^{1}\left(0, T ; L^{1}(\Omega)\right)}+\left\|r\left(\theta_{n}\right)\right\|_{L^{1}\left(0, T ; L^{1}(\Omega)\right)} \\
& +\left\|\theta_{\delta_{n}}^{b}\right\|_{L^{1}((0, T) \times \omega)}+\frac{1}{2}\|c\|_{L^{1}(\Omega)}+\left\|c \theta_{\delta_{n}}^{0}\right\|_{L^{1}(\Omega)}
\end{aligned}
$$

and

$$
\begin{aligned}
& Q_{0}=\left\{(t, x) \in(0, T) \times \Omega ;\left|\theta_{n}(t, x)\right| \leq 1\right\}, \\
& Q_{k}=\left\{(t, x) \in(0, T) \times \Omega ; k<\left|\theta_{n}(t, x)\right| \leq k+1\right\} \quad \text { for all } k \in \mathbb{N}^{*} .
\end{aligned}
$$

With (4.13), we have

$$
\int_{Q_{k}}\left\|\nabla \theta_{n}\right\|^{2} d x d t \leq \frac{C_{\delta_{n}}}{k_{0}} \quad \forall k \in \mathbb{N}
$$

Since $q \in\left(1, \frac{5}{4}\right)$, we may use Hölder's inequality, which yields

$$
\begin{aligned}
\int_{Q_{k}}\left\|\nabla \theta_{n}\right\|^{q} d x d t & \leq\left(\int_{Q_{k}}\left\|\nabla \theta_{n}\right\|^{2} d x d t\right)^{q / 2}\left(\operatorname{meas}\left(Q_{k}\right)\right)^{1-\frac{q}{2}} \\
& \leq\left(\frac{C_{\delta_{n}}}{k_{0}}\right)^{q / 2}\left(\operatorname{meas}\left(Q_{k}\right)\right)^{\frac{2-q}{2}} \quad \forall k \in \mathbb{N} .
\end{aligned}
$$

Then

$$
\begin{aligned}
\int_{0}^{T} \int_{\Omega}\left\|\nabla \theta_{n}\right\|^{q} d x d t= & \int_{\bigcup_{k \geq 0} Q_{k}}\left\|\nabla \theta_{n}\right\|^{q} d x d t \leq\left(\frac{C_{\delta_{n}}}{k_{0}}\right)^{q / 2}(T \operatorname{meas}(\Omega))^{\frac{2-q}{2}} \\
& +\sum_{k \geq 1}\left(\frac{C_{\delta_{n}}}{k_{0}}\right)^{q / 2} \frac{1}{k^{r \frac{2-q}{2}}}\left(\int_{Q_{k}}\left|\theta_{n}\right|^{r} d x d t\right)^{\frac{2-q}{2}}
\end{aligned}
$$

and using again Hölder's inequality

$$
\begin{aligned}
\int_{0}^{T} \int_{\Omega}\left\|\nabla \theta_{n}\right\|^{q} d x d t \leq & \left(\frac{C_{\delta_{n}}}{k_{0}}\right)^{q / 2}(T \operatorname{meas}(\Omega))^{\frac{2-q}{2}} \\
& +\left(\frac{C_{\delta_{n}}}{k_{0}}\right)^{q / 2}\left(\sum_{k \geq 1} \frac{1}{k^{r \frac{2-q}{q}}}\right)^{q / 2}\left(\int_{0}^{T} \int_{\Omega}\left|\theta_{n}\right|^{r} d x d t\right)^{\frac{2-q}{2}}
\end{aligned}
$$

for all $r \geq 1$ such that $r \frac{2-q}{q}>1$. Next, we choose $r=\frac{4}{3} q$. It follows that $\frac{q^{*}(1-r)}{1-q^{*}}=q$, where $q^{*}=\frac{3 q}{3-q}$ and

$$
\int_{0}^{T} \int_{\Omega}\left|\theta_{n}\right|^{r} d x d t \leq \int_{0}^{T}\left(\int_{\Omega}\left|\theta_{n}\right| d x\right)^{\frac{r}{4}}\left(\int_{\Omega}\left|\theta_{n}\right|^{q^{*}} d x\right)^{\frac{3 r}{4 q^{*}}} d t .
$$


Then (4.12) implies that

$$
\begin{aligned}
\left\|\theta_{n}\right\|_{L^{r}\left(0, T ; L^{r}(\Omega)\right)}^{r} & =\int_{0}^{T} \int_{\Omega}\left|\theta_{n}\right|^{r} d x d t \\
& \leq\left(\frac{C_{\delta_{n}}}{c_{0}}\right)^{\frac{r}{4}} \int_{0}^{T}\left(\int_{\Omega}\left|\theta_{n}\right|^{q^{*}} d x\right)^{\frac{q}{q^{*}}} d t=\left(\frac{C_{\delta_{n}}}{c_{0}}\right)^{\frac{r}{4}}\left\|\theta_{n}\right\|_{L^{q}\left(0, T ; L^{q^{*}}(\Omega)\right)}^{q} .
\end{aligned}
$$

Going back to (4.14),

$$
\begin{aligned}
\int_{0}^{T}\left\|\theta_{n}\right\|_{W_{\Gamma_{1} \cup \Gamma_{L}}^{1, q}(\Omega)}^{q} d t & =\int_{0}^{T} \int_{\Omega}\left\|\nabla \theta_{n}\right\|^{q} d x d t \\
\leq & \left(\frac{C_{\delta_{n}}}{k_{0}}\right)^{q / 2}(T \operatorname{meas}(\Omega))^{\frac{2-q}{2}} \\
& +\left(\frac{C_{\delta_{n}}}{k_{0}}\right)^{q / 2}\left(\sum_{k \geq 1} \frac{1}{k^{r \frac{2-q}{q}}}\right)^{q / 2}\left(\frac{C_{\delta_{n}}}{c_{0}}\right)^{\frac{r}{4} \frac{2-q}{2}}\left\|\theta_{n}\right\|_{L^{q\left(0, T ; L q^{*}(\Omega)\right)}}^{q \frac{2-q}{2}}
\end{aligned}
$$

Owing to that $W^{1, q}(\Omega)$ is continuously embedded into $L^{q^{*}}(\Omega)$, we obtain

$$
\begin{aligned}
\left\|\theta_{n}\right\|_{L^{q}\left(0, T ; L^{\left.q^{*}(\Omega)\right)}\right.}^{q}= & \int_{0}^{T}\left\|\theta_{n}\right\|_{L^{q^{*}(\Omega)}}^{q} d t \\
\leq & \left(C_{q}^{\prime}\right)^{q}\left(\left(\frac{C_{\delta_{n}}}{k_{0}}\right)^{q / 2}(T \operatorname{meas}(\Omega))^{\frac{2-q}{2}}\right. \\
& \left.+\left(\frac{C_{\delta_{n}}}{k_{0}}\right)^{q / 2}\left(\sum_{k \geq 1} \frac{1}{k^{r \frac{2-q}{q}}}\right)^{q / 2}\left(\frac{C_{\delta_{n}}}{c_{0}}\right)^{\frac{r}{4} \frac{2-q}{2}}\left\|\theta_{n}\right\|_{L^{q}\left(0, T ; L^{q^{*}}(\Omega)\right)}^{\frac{2-q}{2}}\right)
\end{aligned}
$$

where $C_{q}^{\prime}$ is the norm of the identity mapping from $W_{\Gamma_{1} \cup \Gamma_{L}}^{1, q}(\Omega)$ into $L^{q^{*}}(\Omega)$. By observing that $\frac{2-q}{2}<1$ and recalling that $r \frac{2-q}{q}=\frac{4}{3}(2-q)>1$, we obtain

$$
\begin{aligned}
\left\|\theta_{n}\right\|_{L^{q}\left(0, T ; L^{\left.q^{*}(\Omega)\right)}\right.}^{q} \leq & \max \left(1,\left(C_{q}^{\prime}\right)^{2}\left(\left(\frac{C_{\delta_{n}}}{k_{0}}\right)^{q / 2}(T \operatorname{meas}(\Omega))^{\frac{2-q}{2}}\right.\right. \\
& \left.\left.+\left(\frac{C_{\delta_{n}}}{k_{0}}\right)^{q / 2}\left(\sum_{k \geq 1} \frac{1}{k^{\frac{4}{3}(2-q)}}\right)^{q / 2}\left(\frac{C_{\delta_{n}}}{c_{0}}\right)^{\frac{q}{3} \frac{2-q}{2}}\right)^{2 / q}\right) .
\end{aligned}
$$

Finally, recalling the definition of $g_{\delta_{n}}$ and using (2.4) and (2.7), we obtain

$$
\begin{aligned}
C_{\delta_{n}}= & \left\|\frac{2 \mu\left(\theta_{n}, \bar{v}_{n}+v^{0} \xi,\left|D\left(\bar{v}_{n}+v^{0} \xi\right)\right|\right)\left|D\left(\bar{v}_{n}+v^{0} \xi\right)\right|^{p}}{1+2 \delta_{n} \mu\left(\theta_{n}, \bar{v}_{n}+v^{0} \xi,\left|D\left(\bar{v}_{n}+v^{0} \xi\right)\right|\right)\left|D\left(\bar{v}_{n}+v^{0} \xi\right)\right|^{p}}\right\|_{L^{1}\left(0, T ; L^{1}(\Omega)\right)} \\
& +\left\|r\left(\theta_{n}\right)\right\|_{L^{1}\left(0, T ; L^{1}(\Omega)\right)}+\left\|\theta_{\delta_{n}}^{b}\right\|_{L^{1}((0, T) \times \omega)}+\frac{1}{2}\|c\|_{L^{1}(\Omega)}+\left\|c \theta_{\delta_{n}}^{0}\right\|_{L^{1}(\Omega)} \\
\leq & 2 \mu_{1}\left\|D\left(\bar{v}_{n}+v^{0} \xi\right)\right\|_{L^{p}\left(0, T ;\left(L^{p}(\Omega)\right)^{3 \times 3}\right)}^{p}+\left(r_{1}+\frac{c_{1}}{2}\right) \operatorname{meas}(\Omega) T \\
& +\left\|\theta^{b}\right\|_{L^{1}((0, T) \times \omega)}+c_{1}\left\|\theta^{0}\right\|_{L^{1}(\Omega)}+\delta_{n}\left(1+c_{1}\right) \\
\leq & 2 \mu_{1}\left(\left\|\bar{v}_{n}\right\|_{L^{p}\left(0, T ; V_{0 . \text { div }}^{p}\right)}+\left\|v^{0} \xi\right\|_{L^{p}\left(0, T ; V_{\Gamma_{1}}^{p}\right)}\right)^{p}+\left(r_{1}+\frac{c_{1}}{2}\right) \operatorname{meas}(\Omega) T
\end{aligned}
$$




$$
+\left\|\theta^{b}\right\|_{L^{1}((0, T) \times \omega)}+c_{1}\left\|\theta^{0}\right\|_{L^{1}(\Omega)}+\frac{1}{n}\left(1+c_{1}\right)
$$

and with (4.6)

$$
\begin{aligned}
C_{\delta_{n}} \leq & 2 \mu_{1}\left(\widetilde{C}^{\text {flow }}+\left\|v^{0} \xi\right\|_{L^{p}\left(0, T ; V_{\Gamma_{1}}^{p}\right)}\right)^{p}+\left(r_{1}+\frac{c_{1}}{2}\right) \operatorname{meas}(\Omega) T \\
& +\left\|\theta^{b}\right\|_{L^{1}((0, T) \times \omega)}+c_{1}\left\|\theta^{0}\right\|_{L^{1}(\Omega)}+\left(1+c_{1}\right)
\end{aligned}
$$

for all $n \geq 1$. Then (4.15)-(4.16) allows us to conclude.

As a corollary, we also obtain an estimate for $\frac{\partial \theta_{n}}{\partial t}$.

Proposition 4.3 (A priori estimate for $\frac{\partial \theta_{n}}{\partial t}$ ) Let $q \in\left(1, \frac{5}{4}\right)$. Under the previous assumptions, we have the following estimate:

$$
\begin{aligned}
\| \frac{\partial \theta_{n}}{\partial t} & \|_{L^{1}\left(0, T ;\left(W_{\Gamma_{1} \cup \Gamma_{L}}^{1, q^{\prime}}(\Omega)\right)^{\prime}\right)} \\
\leq & \frac{1}{c_{0}} C_{q^{\prime}}\left(2 \mu_{1}\left(\widetilde{C}^{\text {flow }}+\left\|v^{0} \xi\right\|_{L^{p}\left(0, T ; V_{\Gamma_{1}}^{p}\right)}\right)^{p}+r_{1} \operatorname{meas}(\Omega) T+\left\|\theta^{b}\right\|_{L^{1}((0, T) \times \omega)}+1\right) \\
& +\frac{T^{1 / q^{\prime}}}{c_{0}^{2}}\|K\|_{\left(L^{\infty}(\Omega)\right)^{3 \times 3}} C_{q}^{\text {heat }}\left(c_{0}+C_{q^{\prime}}\|\nabla c\|_{L^{q^{\prime}}(\Omega)}\right),
\end{aligned}
$$

where $C_{q^{\prime}}$ is the norm of the continuous injection of $W_{\Gamma_{1} \cup \Gamma_{L}}^{1, q^{\prime}}(\Omega)$ into $C^{0}(\bar{\Omega})$.

Proof Since $q \in\left(1, \frac{5}{4}\right)$, we have $q^{\prime}=\frac{q}{q-1}>5$, and $W_{\Gamma_{1} \cup \Gamma_{L}}^{1, q^{\prime}}(\Omega)$ is continuously embedded into $W_{\Gamma_{1} \cup \Gamma_{L}}^{1,2}(\Omega)$ and into $C^{0}(\bar{\Omega})$. Moreover, owing to that $c \in W^{1, q^{\prime}}(\Omega)$, we also have $\frac{w}{c} \in$ $W_{\Gamma_{1} \cup \Gamma_{L}}^{1, q^{\prime}}(\Omega) \subset W_{\Gamma_{1} \cup \Gamma_{L}}^{1,2}(\Omega)$ for all $w \in W_{\Gamma_{1} \cup \Gamma_{L}}^{1, q^{\prime}}(\Omega)$. Let $\psi=w \widetilde{\zeta}$ with $w \in W_{\Gamma_{1} \cup \Gamma_{L}}^{1, q^{\prime}}(\Omega)$ and $\tilde{\zeta} \in$ $\mathcal{D}(0, T)$. With $\widetilde{w}=\frac{\psi}{c}=\frac{w}{c} \zeta$ in $(4.11)$, we get

$$
\begin{aligned}
& \left|\int_{0}^{T} \int_{\Omega} \frac{\partial \theta_{n}}{\partial t} \psi d x d t\right| \\
& \leq\left|\int_{0}^{T} \int_{\Omega} \frac{1}{c}\left(K \nabla \theta_{n}\right) \cdot \nabla \psi d x d t\right| \\
& \quad+\left|\int_{0}^{T} \int_{\Omega} \frac{\psi}{c^{2}}\left(K \nabla \theta_{n}\right) \cdot \nabla c d x d t\right|+\left|\int_{0}^{T} \int_{\Omega} \frac{1}{c}\left(g_{\delta_{n}}\left(\theta_{n}, \bar{v}_{n}\right)+r\left(\theta_{n}\right)\right) \psi d x d t\right| \\
& \quad+\left|\int_{0}^{T} \int_{\omega} \frac{1}{c} \theta_{\delta_{n}}^{b} \psi d x^{\prime} d t\right| .
\end{aligned}
$$

Recalling that $\theta_{n} \in W^{1,2}((0, T) \times \Omega)$, the same equality remains true for all $\psi \in L^{2}(0, T$; $\left.W_{\Gamma_{1} \cup \Gamma_{L}}^{1, q^{\prime}}(\Omega)\right)$. Let us now consider $\psi \in L^{\infty}\left(0, T ; W_{\Gamma_{1} \cup \Gamma_{L}}^{1, q^{\prime}}(\Omega)\right)$. We obtain

$$
\begin{aligned}
& \left|\int_{0}^{T} \int_{\Omega} \frac{\partial \theta_{n}}{\partial t} \psi d x d t\right| \\
& \quad \leq \frac{T^{1 / q^{\prime}}}{c_{0}}\|K\|_{\left(L^{\infty}(\Omega)\right)^{3 \times 3}}\left\|\nabla \theta_{n}\right\|_{L^{q}\left(0, T ; \mathbf{L}^{q}(\Omega)\right)}\|\nabla \psi\|_{L^{\infty}\left(0, T ; \mathbf{L}^{q^{\prime}}(\Omega)\right)}
\end{aligned}
$$




$$
\begin{aligned}
& +\frac{T^{1 / q^{\prime}}}{c_{0}^{2}}\|K\|_{\left(L^{\infty}(\Omega)\right)^{3 \times 3}}\left\|\nabla \theta_{n}\right\|_{L^{q}\left(0, T ; \mathbf{L}^{q}(\Omega)\right)}\|\psi\|_{L^{\infty}\left(0, T ; C^{0}(\bar{\Omega})\right)}\|\nabla c\|_{\mathbf{L}^{q^{\prime}(\Omega)}} \\
& +\frac{1}{c_{0}}\left(\left\|g_{\delta_{n}}\left(\theta_{n}, \bar{v}_{n}\right)+r\left(\theta_{n}\right)\right\|_{L^{1}\left(0, T ; L^{1}(\Omega)\right)}+\left\|\theta_{\delta_{n}}^{b}\right\|_{L^{1}((0, T) \times \omega)}\right)\|\psi\|_{L^{\infty}\left(0, T ; C^{0}(\bar{\Omega})\right)} \\
& \leq \frac{T^{1 / q^{\prime}}}{c_{0}}\|K\|_{\left(L^{\infty}(\Omega)\right)^{3 \times 3}}\left\|\nabla \theta_{n}\right\|_{L^{q}\left(0, T ; \mathbf{L}^{q}(\Omega)\right)}\|\nabla \psi\|_{L^{\infty}\left(0, T ; \mathbf{L}^{q^{\prime}}(\Omega)\right)} \\
& +\frac{T^{1 / q^{\prime}}}{c_{0}^{2}}\|K\|_{\left(L^{\infty}(\Omega)\right)^{3 \times 3}}\left\|\nabla \theta_{n}\right\|_{L^{q}\left(0, T ; \mathbf{L}^{q}(\Omega)\right)}\|\psi\|_{L^{\infty}\left(0, T ; C^{0}(\bar{\Omega})\right)}\|\nabla c\|_{\mathbf{L}^{q^{\prime}(\Omega)}} \\
& +\frac{1}{c_{0}}\left(2 \mu_{1}\left(\widetilde{C}^{\text {flow }}+\left\|v^{0} \xi\right\|_{L^{p}\left(0, T ; V_{\Gamma_{1}}^{p}\right.}\right)^{p}+r_{1} \operatorname{meas}(\Omega) T+\left\|\theta^{b}\right\|_{L^{1}((0, T) \times \omega)}+1\right) \\
& \quad \times\|\psi\|_{L^{\infty}\left(0, T ; C^{0}(\bar{\Omega})\right)}
\end{aligned}
$$

which allows us to conclude.

It follows that, by possibly extracting a subsequence still denoted $\left(\bar{v}_{n}, \pi_{n}, \theta_{n}\right)_{n \geq 1}$, there exists a triplet $(\bar{v}, \pi, \theta)$ such that

$$
\begin{gathered}
\bar{v}_{n} \longrightarrow \bar{v} \quad \text { weakly in } L^{p}\left(0, T ; V_{0 . \mathrm{div}}^{p}\right) \text { and } \\
\text { weakly* in } L^{\infty}\left(0, T ; \mathbf{L}^{2}(\Omega)\right), \\
\frac{\partial \bar{v}_{n}}{\partial t} \longrightarrow \frac{\partial \bar{v}}{\partial t} \quad \text { weakly in } L^{p^{\prime}}\left(0, T ;\left(V_{0 . \mathrm{div}}^{p}\right)^{\prime}\right), \\
\pi_{n} \longrightarrow \pi \quad \text { weakly* in } H^{-1}\left(0, T ; L_{0}^{p^{\prime}}(\Omega)\right),
\end{gathered}
$$

and

$$
\theta_{n} \longrightarrow \theta \quad \text { weakly in } L^{q}\left(0, T ; W_{\Gamma_{1} \cup \Gamma_{L}}^{1, q}(\Omega)\right)
$$

Moreover, with the Aubin and Simon lemmas [29, 34], by possibly extracting another subsequence still denoted $\left(\bar{v}_{n}, \pi_{n}, \theta_{n}\right)_{n \geq 1}$, we have

$$
\begin{aligned}
\bar{v}_{n} \longrightarrow \bar{v} & \text { strongly in } C^{0}([0, T] ; H) \\
& \text { and in } L^{p}\left(0, T ; \mathbf{L}^{p}(\Omega)\right),
\end{aligned}
$$

where we recall that $H=\left\{\psi \in \mathbf{L}^{2}(\Omega) ; \operatorname{div}(\psi)=0\right.$ in $\Omega, \psi \cdot n=0$ on $\left.\partial \Omega\right\}$, and with Aubin's generalized lemma [29], we also have

$$
\theta_{n} \longrightarrow \theta \quad \text { strongly in } L^{q}\left(0, T ; L^{q}(\Omega)\right)
$$

We infer that

$$
\bar{v}_{n}(0, \cdot) \longrightarrow \bar{v}(0, \cdot)=0 \quad \text { strongly in } \mathbf{L}^{2}(\Omega)
$$

Furthermore, by possibly extracting again a subsequence still denoted $\left(\bar{v}_{n}, \pi_{n}, \theta_{n}\right)_{n \geq 1}$, we have

$$
\bar{v}_{n} \longrightarrow \bar{v}, \quad \theta_{n} \longrightarrow \theta \quad \text { a.e. in }(0, T) \times \Omega .
$$


These convergence properties do not allow us to pass directly to the limit as $n$ tends to $+\infty$, and we need a better convergence result for $\left(\bar{v}_{n}\right)_{n \geq 1}$ to overcome the difficulty due to the non-linearity of the mapping $\mathcal{F}$.

Proposition 4.4 Under the previous assumptions, $\left(D\left(\bar{v}_{n}\right)\right)_{n \geq 1}$ converges strongly to $D(\bar{v})$ in $L^{p}\left(0, T ;\left(L^{p}(\Omega)\right)^{3 \times 3}\right)$.

Proof Let $(\hat{v}, \hat{\pi})$ be the unique solution of the problem $\left(P_{(\bar{v}, \theta)}^{\text {flow }}\right)$. We have

$$
\begin{aligned}
& \int_{0}^{T}\left\langle\frac{\partial \hat{v}}{\partial t}, \bar{\varphi}-\left.\hat{v}\right|_{\left(V_{0 . \text { div }}^{p}\right)^{\prime}, V_{0 . \text { div }}^{p}} d t\right. \\
& \quad+\int_{0}^{T} \int_{\Omega} \mathcal{F}\left(\theta, \bar{v}+v^{0} \xi, D\left(\hat{v}+v^{0} \xi\right)\right): D(\bar{\varphi}-\hat{v}) d x d t \\
& \quad+J(\bar{\varphi})-J(\hat{v}) \geq \int_{0}^{T}\left(f+v^{0} \frac{\partial \xi}{\partial t}, \bar{\varphi}-\hat{v}\right)_{\mathbf{L}^{2}(\Omega)} d t \quad \forall \bar{\varphi} \in L^{p}\left(0, T ; V_{0 . \text { div }}^{p}\right) .
\end{aligned}
$$

For all $n \geq 1$, we also have

$$
\begin{aligned}
& \int_{0}^{T}\left\langle\frac{\partial \bar{v}_{n}}{\partial t}, \bar{\varphi}-\bar{v}_{n}\right\rangle_{\left(V_{0 . \text { div }}^{p}\right)^{\prime}, V_{0 . \text { div }}^{p}} d t \\
& \quad+\int_{0}^{T} \int_{\Omega} \mathcal{F}\left(\theta_{n}, \bar{v}_{n}+v^{0} \xi, D\left(\bar{v}_{n}+v^{0} \xi\right)\right): D\left(\bar{\varphi}-\bar{v}_{n}\right) d x d t \\
& \quad+J(\bar{\varphi})-J\left(\bar{v}_{n}\right) \geq \int_{0}^{T}\left(f+v^{0} \frac{\partial \xi}{\partial t}, \bar{\varphi}-\bar{v}_{n}\right)_{\mathbf{L}^{2}(\Omega)} d t \quad \forall \bar{\varphi} \in L^{p}\left(0, T ; V_{0 . \text { div }}^{p}\right) .
\end{aligned}
$$

Let us choose $\bar{\varphi}=\hat{v}$ in (4.26) and $\bar{\varphi}=\bar{v}_{n}$ in (4.25). By adding the two inequalities, we obtain:

$$
\begin{aligned}
& \int_{0}^{T}\left\langle\frac{\partial\left(\bar{v}_{n}-\hat{v}\right)}{\partial t}, \bar{v}_{n}-\hat{v}\right\rangle_{\left(V_{0 \text { div }}^{p}\right)^{\prime}, V_{0 . \text { div }}^{p}} d t \\
& \quad+\int_{0}^{T} \int_{\Omega}\left(\mathcal{F}\left(\theta_{n}, \bar{v}_{n}+v^{0} \xi, D\left(\bar{v}_{n}+v^{0} \xi\right)\right)\right. \\
&\left.\quad-\mathcal{F}\left(\theta, \bar{v}+v^{0} \xi, D\left(\hat{v}+v^{0} \xi\right)\right)\right): D\left(\bar{v}_{n}-\hat{v}\right) d x d t \leq 0
\end{aligned}
$$

and thus,

$$
\begin{aligned}
\frac{1}{2}\left\|\bar{v}_{n}-\hat{v}\right\|_{\mathbf{L}^{2}(\Omega)}^{2}(T) & \\
& +\int_{0}^{T} \int_{\Omega}\left(\mathcal{F}\left(\theta_{n}, \bar{v}_{n}+v^{0} \xi, D\left(\bar{v}_{n}+v^{0} \xi\right)\right)\right. \\
& \left.-\mathcal{F}\left(\theta_{n}, \bar{v}_{n}+v^{0} \xi, D\left(\hat{v}+v^{0} \xi\right)\right)\right): D\left(\bar{v}_{n}-\hat{v}\right) d x d t \\
\leq & \int_{0}^{T} \int_{\Omega}\left(\mathcal{F}\left(\theta, \bar{v}+v^{0} \xi, D\left(\hat{v}+v^{0} \xi\right)\right)\right. \\
& \left.\quad-\mathcal{F}\left(\theta_{n}, \bar{v}_{n}+v^{0} \xi, D\left(\hat{v}+v^{0} \xi\right)\right)\right): D\left(\bar{v}_{n}-\hat{v}\right) d x d t .
\end{aligned}
$$


We perform the same kind of computations as in Theorem 3.5. More precisely, we split the second term of the left-hand side as follows:

$$
\begin{aligned}
\int_{0}^{T} & \int_{\Omega}\left(\mathcal{F}\left(\theta_{n}, \bar{v}_{n}+v^{0} \xi, D\left(\bar{v}_{n}+v^{0} \xi\right)\right)\right. \\
& \left.-\mathcal{F}\left(\theta_{n}, \bar{v}_{n}+v^{0} \xi, D\left(\hat{v}+v^{0} \xi\right)\right)\right): D\left(\bar{v}_{n}-\hat{v}\right) d x d t \\
= & \int_{0}^{T} \int_{\Omega}\left(\mathcal{F}_{1}\left(D\left(\bar{v}_{n}+v^{0} \xi\right)\right)-\mathcal{F}_{1}\left(D\left(\hat{v}+v^{0} \xi\right)\right)\right): D\left(\bar{v}_{n}-\hat{v}\right) d x d t \\
& +\int_{0}^{T} \int_{\Omega}\left(\mathcal{F}_{2}\left(\theta_{n}, \bar{v}_{n}+v^{0} \xi, D\left(\bar{v}_{n}+v^{0} \xi\right)\right)\right. \\
& \left.-\mathcal{F}_{2}\left(\theta_{n}, \bar{v}_{n}+v^{0} \xi, D\left(\hat{v}+v^{0} \xi\right)\right)\right): D\left(\bar{v}_{n}-\hat{v}\right) d x d t,
\end{aligned}
$$

where we recall that

$$
\begin{aligned}
& \mathcal{F}_{1}\left(\lambda_{2}\right)=\mu_{0}\left\|\lambda_{2}\right\|^{p-2} \lambda_{2} \quad \text { if } \lambda_{2} \neq 0_{\mathbb{R}^{3 \times 3}}, \quad \mathcal{F}_{1}\left(\lambda_{2}\right)=0_{\mathbb{R}^{3 \times 3}} \quad \text { otherwise, } \\
& \begin{cases}\mathcal{F}_{2}\left(\lambda_{0}, \lambda_{1}, \lambda_{2}\right)=2 \bar{\mu}\left(\lambda_{0}, \lambda_{1},\left\|\lambda_{2}\right\|\right)\left\|\lambda_{2}\right\|^{p-2} \lambda_{2} \quad \text { if } \lambda_{2} \neq 0_{\mathbb{R}^{3 \times 3}}, \\
\mathcal{F}_{2}\left(\lambda_{0}, \lambda_{1}, \lambda_{2}\right)=0_{\mathbb{R}^{3 \times 3}} \quad \text { otherwise }\end{cases}
\end{aligned}
$$

and $\bar{\mu}=\mu-\frac{\mu_{0}}{2}$. Since $\lambda_{2} \mapsto \mathcal{F}_{2}\left(\cdot, \cdot, \lambda_{2}\right)$ is monotone in $\mathbb{R}^{3 \times 3}$ (see Lemma 1 in [9]), we have

$$
\begin{aligned}
\int_{0}^{T} & \int_{\Omega}\left(\mathcal{F}_{2}\left(\theta_{n}, \bar{v}_{n}+v^{0} \xi, D\left(\bar{v}_{n}+v^{0} \xi\right)\right)\right. \\
& \left.-\mathcal{F}_{2}\left(\theta_{n}, \bar{v}_{n}+v^{0} \xi, D\left(\hat{v}+v^{0} \xi\right)\right)\right): D\left(\bar{v}_{n}-\hat{v}\right) d x d t \geq 0,
\end{aligned}
$$

and (4.27) yields

$$
\begin{aligned}
\frac{1}{2} \| \bar{v}_{n} & -\hat{v} \|_{\mathbf{L}^{2}(\Omega)}^{2}(T) \\
& +\int_{0}^{T} \int_{\Omega}\left(\mathcal{F}_{1}\left(D\left(\bar{v}_{n}+v^{0} \xi\right)\right)-\mathcal{F}_{1}\left(D\left(\hat{v}+v^{0} \xi\right)\right)\right): D\left(\bar{v}_{n}-\hat{v}\right) d x d t \\
\leq & \int_{0}^{T} \int_{\Omega}\left(\mathcal{F}_{2}\left(\theta, \bar{v}+v^{0} \xi, D\left(\hat{v}+v^{0} \xi\right)\right)\right. \\
& \left.-\mathcal{F}_{2}\left(\theta_{n}, \bar{v}_{n}+v^{0} \xi, D\left(\hat{v}+v^{0} \xi\right)\right)\right): D\left(\bar{v}_{n}-\hat{v}\right) d x d t .
\end{aligned}
$$

Then we distinguish two cases.

Case 1: $p \in[6 / 5,2)$

Recalling that

$$
\left(\|\lambda\|^{p}+\left\|\lambda^{\prime}\right\|^{p}\right)^{\frac{2-p}{2}}\left(\left(\mathcal{F}_{1}(\lambda)-\mathcal{F}_{1}\left(\lambda^{\prime}\right)\right):\left(\lambda-\lambda^{\prime}\right)\right)^{\frac{p}{2}} \geq \frac{\left(\mu_{0}(p-1)\right)^{\frac{p}{2}}}{2^{\frac{(p-1)(2-p)}{2}}}\left\|\lambda-\lambda^{\prime}\right\|^{p}
$$


for all $\left(\lambda, \lambda^{\prime}\right) \in \mathbb{R}^{3 \times 3} \times \mathbb{R}^{3 \times 3}$, we obtain

$$
\begin{aligned}
& \frac{\left(\mu_{0}(p-1)\right)^{\frac{p}{2}}}{2^{\frac{(p-1)(2-p)}{2}}} \int_{0}^{T} \int_{\Omega}\left\|D\left(\bar{v}_{n}-\hat{v}\right)\right\|^{p} d x d t \\
& \leq\left(\int_{0}^{T} \int_{\Omega}\left(\mathcal{F}_{1}\left(D\left(\bar{v}_{n}+v^{0} \xi\right)\right)-\mathcal{F}_{1}\left(D\left(\hat{v}+v^{0} \xi\right)\right)\right): D\left(\bar{v}_{n}-\hat{v}\right) d x d t\right)^{\frac{p}{2}} \\
& \quad \times\left(\int_{0}^{T} \int_{\Omega}\left(\left\|D\left(\bar{v}_{n}+v^{0} \xi\right)\right\|^{p}+\left\|D\left(\hat{v}+v^{0} \xi\right)\right\|^{p}\right) d x d t\right)^{\frac{2-p}{2}} \\
& \leq\left(\int_{0}^{T} \int_{\Omega}\left(\mathcal{F}_{1}\left(D\left(\bar{v}_{n}+v^{0} \xi\right)\right)-\mathcal{F}_{1}\left(D\left(\hat{v}+v^{0} \xi\right)\right)\right): D\left(\bar{v}_{n}-\hat{v}\right) d x d t\right)^{\frac{p}{2}} \\
& \quad \times\left(\left\|\bar{v}_{n}+v^{0} \xi\right\|_{L^{p}\left(0, T ; V_{\Gamma_{1}}^{p}\right)}^{p}+\left\|\hat{v}+v^{0} \xi\right\|_{L^{p}\left(0, T ; V_{\Gamma_{1}}^{p}\right)}^{p}\right)^{\frac{2-p}{2}} .
\end{aligned}
$$

Thus with (3.11) and (4.6), we get

$$
\begin{aligned}
& \frac{\left(\mu_{0}(p-1)\right)^{\frac{p}{2}}}{2^{\frac{(p-1)(2-p)}{2}}}\left\|D\left(\bar{v}_{n}-\hat{v}\right)\right\|_{L^{p}\left(0, T ;\left(L^{p}(\Omega)\right)^{3 \times 3}\right)}^{p} \\
& \leq\left(\int_{0}^{T} \int_{\Omega}\left(\mathcal{F}_{1}\left(D\left(\bar{v}_{n}+v^{0} \xi\right)\right)-\mathcal{F}_{1}\left(D\left(\hat{v}+v^{0} \xi\right)\right)\right): D\left(\bar{v}_{n}-\hat{v}\right) d x d t\right)^{\frac{p}{2}} \\
& \quad \times\left(\left(\widetilde{C}^{\text {flow }}+\left\|v^{0} \xi\right\|_{L^{p}\left(0, T ; V_{\Gamma_{1}}^{p}\right)}\right)^{p}+\left(C^{\text {flow }}+\left\|v^{0} \xi\right\|_{L^{p}\left(0, T ; V_{\Gamma_{1}}^{p}\right)}\right)^{p}\right)^{\frac{2-p}{2}}
\end{aligned}
$$

By observing that $\widetilde{C}^{\text {flow }} \geq C^{\text {flow }}$, we have finally

$$
\begin{aligned}
\frac{\mu_{0}(p-1)}{2^{(2-p)}\left(\widetilde{C}^{\text {flow }}+\left\|v^{0} \xi\right\|_{L^{p}\left(0, T ; V_{\Gamma_{1}}^{p}\right)}\right)^{(2-p)}}\left\|D\left(\bar{v}_{n}-\hat{v}\right)\right\|_{L^{p}\left(0, T ;\left(L^{p}(\Omega)\right)^{3 \times 3}\right)}^{2} \\
\leq \int_{0}^{T} \int_{\Omega}\left(\mathcal{F}_{1}\left(D\left(\bar{v}_{n}+v^{0} \xi\right)\right)-\mathcal{F}_{1}\left(D\left(\hat{v}+v^{0} \xi\right)\right)\right): D\left(\bar{v}_{n}-\hat{v}\right) d x d t \\
\leq \int_{0}^{T} \int_{\Omega}\left(\mathcal{F}_{2}\left(\theta, \bar{v}+v^{0} \xi, D\left(\hat{v}+v^{0} \xi\right)\right)\right. \\
\left.\quad-\mathcal{F}_{2}\left(\theta_{n}, \bar{v}_{n}+v^{0} \xi, D\left(\hat{v}+v^{0} \xi\right)\right)\right): D\left(\bar{v}_{n}-\hat{v}\right) d x d t \\
\leq \| \mathcal{F}_{2}\left(\theta, \bar{v}^{0}+v^{0} \xi, D\left(\hat{v}+v^{0} \xi\right)\right) \\
\quad-\mathcal{F}_{2}\left(\theta_{n}, \bar{v}_{n}+v^{0} \xi, D\left(\hat{v}+v^{0} \xi\right)\right) \|_{L^{p^{\prime}}\left(0, T ;\left(L^{p^{\prime}}(\Omega)\right)^{3 \times 3}\right)} \\
\quad \times\left\|D\left(\bar{v}_{n}-\hat{v}\right)\right\|_{L^{p}\left(0, T ;\left(L^{p}(\Omega)\right)^{3 \times 3}\right)}
\end{aligned}
$$

which yields

$$
\begin{gathered}
\frac{\mu_{0}(p-1)}{2^{(2-p)}\left(\widetilde{C}^{\text {flow }}+\left\|v^{0} \xi\right\|_{L^{p}\left(0, T ; V_{\Gamma_{1}}^{p}\right)}\right)^{(2-p)}}\left\|D\left(\bar{v}_{n}-\hat{v}\right)\right\|_{L^{p}\left(0, T ;\left(L^{p}(\Omega)\right)^{3 \times 3}\right)} \\
\leq \| \mathcal{F}_{2}\left(\theta, \bar{v}+v^{0} \xi, D\left(\hat{v}+v^{0} \xi\right)\right) \\
-\mathcal{F}_{2}\left(\theta_{n}, \bar{v}_{n}+v^{0} \xi, D\left(\hat{v}+v^{0} \xi\right)\right) \|_{L^{p^{\prime}}\left(0, T ;\left(L^{p^{\prime}}(\Omega)\right)^{3 \times 3}\right)^{*}}
\end{gathered}
$$


Case 2: $p \in[2,+\infty)$

Recalling that

$$
\left(\mathcal{F}_{1}(\lambda)-\mathcal{F}_{1}\left(\lambda^{\prime}\right)\right):\left(\lambda-\lambda^{\prime}\right) \geq \frac{\mu_{0}}{2^{p-1}}\left\|\lambda-\lambda^{\prime}\right\|^{p}
$$

for all $\left(\lambda, \lambda^{\prime}\right) \in \mathbb{R}^{3 \times 3} \times \mathbb{R}^{3 \times 3}$, we obtain

$$
\begin{aligned}
\frac{\mu_{0}}{2^{p-1}}\left\|D\left(\bar{v}_{n}-\hat{v}\right)\right\|_{L^{p}\left(0, T ;\left(L^{p}(\Omega)\right)^{3 \times 3}\right)}^{p} & \leq \int_{0}^{T} \int_{\Omega}\left(\mathcal{F}_{1}\left(D\left(\bar{v}_{n}+v^{0} \xi\right)\right)-\mathcal{F}_{1}\left(D\left(\hat{v}+v^{0} \xi\right)\right)\right): D\left(\bar{v}_{n}-\hat{v}\right) d x d t \\
\leq & \int_{0}^{T} \int_{\Omega}\left(\mathcal{F}_{2}\left(\theta, \bar{v}+v^{0} \xi, D\left(\hat{v}+v^{0} \xi\right)\right)\right. \\
& \left.-\mathcal{F}_{2}\left(\theta_{n}, \bar{v}_{n}+v^{0} \xi, D\left(\hat{v}+v^{0} \xi\right)\right)\right): D\left(\bar{v}_{n}-\hat{v}\right) d x d t \\
\leq & \| \mathcal{F}_{2}\left(\theta, \bar{v}+v^{0} \xi, D\left(\hat{v}+v^{0} \xi\right)\right) \\
& \quad-\mathcal{F}_{2}\left(\theta_{n}, \bar{v}_{n}+v^{0} \xi, D\left(\hat{v}+v^{0} \xi\right)\right) \|_{L^{p^{\prime}}\left(0, T ;\left(L^{p^{\prime}}(\Omega)\right)^{3 \times 3}\right)} \\
& \times\left\|D\left(\bar{v}_{n}-\hat{v}\right)\right\|_{L^{p}\left(0, T ;\left(L^{p}(\Omega)\right)^{3 \times 3}\right)}
\end{aligned}
$$

which yields

$$
\begin{aligned}
\frac{\mu_{0}}{2^{p-1}} & \left\|D\left(\bar{v}_{n}-\hat{v}\right)\right\|_{L^{p}\left(0, T ;\left(L^{p}(\Omega)\right)^{3 \times 3}\right)}^{p-1} \\
\leq & \| \mathcal{F}_{2}\left(\theta, \bar{v}+v^{0} \xi, D\left(\hat{v}+v^{0} \xi\right)\right) \\
& \quad-\mathcal{F}_{2}\left(\theta_{n}, \bar{v}_{n}+v^{0} \xi, D\left(\hat{v}+v^{0} \xi\right)\right) \|_{L^{p^{\prime}}\left(0, T ;\left(L^{p^{\prime}}(\Omega)\right)^{3 \times 3}\right)}
\end{aligned}
$$

By using the convergence properties (4.24) for the sequences $\left(\theta_{n}\right)_{n \geq 1}$ and $\left(\bar{v}_{n}\right)_{n \geq 1}$ and the continuity and boundedness assumptions (2.8) and (2.10) for the mapping $\mu$, we obtain

$$
\begin{aligned}
& \mathcal{F}_{2}\left(\theta_{n}, \bar{v}_{n}+v^{0} \xi, D\left(\hat{v}+v^{0} \xi\right)\right) \longrightarrow \mathcal{F}_{2}\left(\theta, \hat{v}+v^{0} \xi, D\left(\hat{v}+v^{0} \xi\right)\right) \\
& \text { strongly in } L^{p^{\prime}}\left(0, T ;\left(L^{p^{\prime}}(\Omega)\right)^{3 \times 3}\right) .
\end{aligned}
$$

Then with (4.29) if $p \in[6 / 5,2)$ and (4.30) if $p \in[2,+\infty)$, we obtain

$$
\lim _{n \rightarrow+\infty}\left\|D\left(\bar{v}_{n}-\hat{v}\right)\right\|_{L^{p}\left(0, T ;\left(L^{p}(\Omega)\right)^{3 \times 3}\right)}=0
$$

Finally, Korn's inequality implies that the sequence $\left(\bar{v}_{n}\right)_{n \geq 1}$ converges strongly to $\hat{v}$ in $L^{p}\left(0, T ; V_{0 \text { div }}^{p}\right)$, which yields $\hat{v}=\bar{v}$.

By possibly extracting another subsequence, still denoted $\left(\bar{v}_{n}, \pi_{n}, \theta_{n}\right)_{n \geq 1}$, we obtain that

$$
D\left(\bar{v}_{n}\right) \longrightarrow D(\bar{v}) \quad \text { a.e. in }(0, T) \times \Omega \text {. }
$$

By using (2.8) and (2.10), we infer from Lebesgue's theorem that

$$
\begin{aligned}
& \mathcal{F}\left(\theta_{n}, \bar{v}_{n}+v^{0} \xi, D\left(\bar{v}_{n}+v^{0} \xi\right)\right) \longrightarrow \mathcal{F}\left(\theta, \bar{v}+v^{0} \xi, D\left(\bar{v}+v^{0} \xi\right)\right) \\
& \text { strongly in } L^{p^{\prime}}\left(0, T ;\left(L^{p^{\prime}}(\Omega)\right)^{3 \times 3}\right)
\end{aligned}
$$


and

$$
\begin{gathered}
g_{\delta_{n}}\left(\theta_{n}, \bar{v}_{n}\right) \longrightarrow 2 \mu\left(\theta, \bar{v}+v^{0} \xi,\left\|D\left(\bar{v}+v^{0} \xi\right)\right\|\right)\left\|D\left(\bar{v}+v_{0} \xi\right)\right\|^{p} \\
=\mathcal{F}\left(\theta, \bar{v}+v^{0} \xi, D\left(\bar{v}+v^{0} \xi\right)\right): D\left(\bar{v}+v^{0} \xi\right)
\end{gathered}
$$

strongly in $L^{1}\left(0, T ; L^{1}(\Omega)\right)$.

\section{Moreover,}

$$
\bar{v}_{n} \longrightarrow \hat{v}=\bar{v} \quad \text { strongly in } L^{p}\left(0, T ; V_{0 . \operatorname{div}}^{p}\right) .
$$

Reminding that the mapping $J$ is continuous on $L^{p}\left(0, T ; V_{0}^{p}\right)$, we get

$$
J\left(\bar{v}_{n}+\widetilde{\vartheta} \zeta\right) \longrightarrow J(\bar{v}+\widetilde{\vartheta} \zeta) \quad \forall \widetilde{\vartheta} \in V_{0}^{p}, \forall \zeta \in \mathcal{D}(0, T)
$$

Finally, by using the continuity and boundedness assumptions (2.6) - (2.7) for the mapping $r$, we also have

$$
r\left(\theta_{n}\right) \longrightarrow r(\theta) \quad \text { strongly in } L^{1}\left(0, T ; L^{1}(\Omega)\right)
$$

It follows that we can pass to the limit in (4.1) and (4.5), which allows us to conclude that the triplet $(\bar{v}, \pi, \theta)$ is a solution to the coupled fluid flow/heat transfer problem $(P)$.

Remark 4.5 We may observe that $\pi=\hat{\pi}$. Indeed,

$$
\begin{aligned}
& \left\langle\frac{\partial}{\partial t}(\bar{v}, \tilde{\vartheta})_{\mathbf{L}^{2}(\Omega)}, \zeta\right\rangle_{\mathcal{D}^{\prime}(0, T), \mathcal{D}(0, T)} \\
& +\int_{0}^{T} \int_{\Omega} \mathcal{F}\left(\theta, \bar{v}+v^{0} \xi, D\left(\bar{v}+v^{0} \xi\right)\right): D(\tilde{\vartheta}) \zeta d x d t \\
& -\left\langle\int_{\Omega} \pi \operatorname{div}(\tilde{\vartheta}) d x, \zeta\right\rangle_{\mathcal{D}^{\prime}(0, T), \mathcal{D}(0, T)}+J(\bar{v}+\tilde{\vartheta} \zeta)-J(\bar{v}) \\
& \geq \int_{0}^{T}\left(f+v^{0} \frac{\partial \xi}{\partial t}, \tilde{\vartheta}\right)_{\mathbf{L}^{2}(\Omega)} \zeta d t \quad \forall \tilde{\vartheta} \in V_{0}^{p}, \forall \zeta \in \mathcal{D}(0, T)
\end{aligned}
$$

and

$$
\begin{aligned}
\left\langle\frac{\partial}{\partial t}(\hat{v}, \tilde{\vartheta})_{\mathbf{L}^{2}(\Omega)}, \zeta\right\rangle_{\mathcal{D}^{\prime}(0, T), \mathcal{D}(0, T)} & \\
& +\int_{0}^{T} \int_{\Omega} \mathcal{F}\left(\theta, \bar{v}+v^{0} \xi, D\left(\hat{v}+v^{0} \xi\right)\right): D(\tilde{\vartheta}) \zeta d x d t \\
& -\left\langle\int_{\Omega} \hat{\pi} \operatorname{div}(\tilde{\vartheta}) d x, \zeta\right\rangle_{\mathcal{D}^{\prime}(0, T), \mathcal{D}(0, T)}+J(\hat{v}+\tilde{\vartheta} \zeta)-J(\hat{v}) \\
\geq & \int_{0}^{T}\left(f+v^{0} \frac{\partial \xi}{\partial t}, \tilde{\vartheta}\right)_{\mathbf{L}^{2}(\Omega)} \zeta d t \quad \forall \tilde{\vartheta} \in V_{0}^{p}, \forall \zeta \in \mathcal{D}(0, T) .
\end{aligned}
$$


Owing to that $\bar{v}=\hat{v}$, we obtain that

$$
\left\langle\int_{\Omega}(\pi-\hat{\pi}) \operatorname{div}(\tilde{\vartheta}) d x, \zeta\right\rangle_{\mathcal{D}^{\prime}(0, T), \mathcal{D}(0, T)}=0 \quad \forall \tilde{\vartheta} \in \mathbf{W}_{0}^{1, p}(\Omega), \forall \zeta \in \mathcal{D}(0, T)
$$

Hence, for any $\varpi \in L_{0}^{p}(\Omega)$, we may choose $\widetilde{\vartheta}=P_{p}(\varpi)$, where $P_{p}$ is the operator introduced in Proposition 4.1, and we get

$$
\left\langle\int_{\Omega}(\pi-\hat{\pi}) \varpi d x, \zeta\right\rangle_{\mathcal{D}^{\prime}(0, T), \mathcal{D}(0, T)}=0
$$

Furthermore, for any $\varpi^{*} \in L^{p}(\Omega)$, we may define $\varpi \in L_{0}^{p}(\Omega)$ by

$$
\varpi=\varpi^{*}-\frac{1}{\operatorname{meas}(\Omega)} \int_{\Omega} \varpi^{*} d x
$$

and since $\pi-\hat{\pi} \in H^{-1}\left(0, T ; L_{0}^{p}(\Omega)\right)$, we have

$$
\begin{aligned}
& \left\langle\int_{\Omega}(\pi-\hat{\pi}) \varpi d x, \zeta\right\rangle_{\mathcal{D}^{\prime}(0, T), \mathcal{D}(0, T)} \\
& =\left\langle\int_{\Omega}(\pi-\hat{\pi}) \varpi^{*} d x, \zeta\right\rangle_{\mathcal{D}^{\prime}(0, T), \mathcal{D}(0, T)} \\
& \left.\quad-\frac{1}{\operatorname{meas}(\Omega)}\left(\int_{\Omega} \varpi^{*} d x\right)\right)\left\langle\int_{\Omega}(\pi-\hat{\pi}) d x, \zeta\right\rangle_{\mathcal{D}^{\prime}(0, T), \mathcal{D}(0, T)} \\
& =\left\langle\int_{\Omega}(\pi-\hat{\pi}) \varpi^{*} d x, \zeta\right\rangle_{\mathcal{D}^{\prime}(0, T), \mathcal{D}(0, T)}=0 \quad \forall \varpi^{*} \in L^{p}(\Omega), \forall \zeta \in \mathcal{D}(0, T),
\end{aligned}
$$

which implies $\pi=\hat{\pi}$.

Remark 4.6 The strong convergence of $\left(g_{\delta_{n}}\left(\theta_{n}, \bar{v}_{n}\right)\right)_{n \geq 1}$ and $\left(r\left(\theta_{n}\right)\right)_{n \geq 1}$ in the space $L^{1}(0, T$; $\left.L^{1}(\Omega)\right)$ implies that $\theta \in C^{0}\left([0, T] ; L^{1}(\Omega)\right)$ and $\theta(0, \cdot)=\theta^{0}$. Indeed, starting from (4.11), we have also

$$
\begin{aligned}
& \int_{0}^{T} \int_{\Omega} c \frac{\partial\left(\theta_{n}-\theta_{n^{\prime}}\right)}{\partial t} \widetilde{w} d x d t+\int_{0}^{T} \int_{\Omega}\left(K \nabla\left(\theta_{n}-\theta_{n^{\prime}}\right)\right) \cdot \nabla \widetilde{w} d x d t \\
& =\int_{0}^{T} \int_{\Omega}\left(g_{\delta_{n}}\left(\theta_{n}, \bar{v}_{n}\right)-g_{\delta_{n^{\prime}}}\left(\theta_{n^{\prime}}, \bar{v}_{n^{\prime}}\right)\right) \widetilde{w} d x d t+\int_{0}^{T} \int_{\Omega}\left(r\left(\theta_{n}\right)-r\left(\theta_{n^{\prime}}\right)\right) \widetilde{w} d x d t \\
& \quad+\int_{0}^{T} \int_{\omega}\left(\theta_{\delta_{n}}^{b}-\theta_{\delta_{n^{\prime}}}^{b}\right) \widetilde{w} d x^{\prime} d t
\end{aligned}
$$

for all $\widetilde{w} \in L^{2}\left(0, T ; W_{\Gamma_{1} \cup \Gamma_{L}}^{1,2}(\Omega)\right)$, for all $n \geq 1$, and $n^{\prime} \geq 1$. By choosing $\widetilde{w}=\psi_{0}^{m}\left(\theta_{n}-\theta_{n^{\prime}}\right) \mathbf{1}_{[0, t]}$, with $t \in(0, T]$ and by passing to the limit, as $m$ tends to $+\infty$, we get

$$
\begin{aligned}
\int_{\Omega} c(x) & \Phi_{0}\left(\theta_{n}(t, x)-\theta_{n^{\prime}}(t, x)\right) d x \\
+ & \int_{0}^{t} \int_{\Omega} \Psi_{0}\left(\theta_{n}(s, x)-\theta_{n^{\prime}}(s, x)\right)
\end{aligned}
$$




$$
\begin{aligned}
& \times\left(K(x) \nabla\left(\theta_{n}(s, x)-\theta_{n^{\prime}}(s, x)\right)\right) \cdot \nabla\left(\theta_{n}(s, x)-\theta_{n^{\prime}}(s, x)\right) d x d s \\
= & \int_{0}^{t} \int_{\Omega}\left(g_{\delta_{n}}\left(\theta_{n}, \bar{v}_{n}\right)-g_{\delta_{n^{\prime}}}\left(\theta_{n^{\prime}}, \bar{v}_{n^{\prime}}\right)\right) \psi_{0}\left(\theta_{n}-\theta_{n^{\prime}}\right) d x d s \\
& +\int_{0}^{t} \int_{\Omega}\left(r\left(\theta_{n}\right)-r\left(\theta_{n^{\prime}}\right)\right) \psi_{0}\left(\theta_{n}-\theta_{n^{\prime}}\right) d x d s \\
& +\int_{0}^{t} \int_{\omega}\left(\theta_{\delta_{n}}^{b}-\theta_{\delta_{n^{\prime}}}^{b}\right) \psi_{0}\left(\theta_{n}-\theta_{n^{\prime}}\right) d x^{\prime} d s+\int_{\Omega} c(x) \Phi_{0}\left(\theta_{\delta_{n}}^{0}-\theta_{\delta_{n^{\prime}}}^{0}\right) d x,
\end{aligned}
$$

which yields

$$
\begin{aligned}
& c_{0} \int_{\Omega} \Phi_{0}\left(\theta_{n}(t, x)-\theta_{n^{\prime}}\right) d x \\
& \quad \leq\left\|g_{\delta_{n}}\left(\theta_{n}, \bar{v}_{n}\right)-g_{\delta_{n^{\prime}}}\left(\theta_{n^{\prime}}, \bar{v}_{n^{\prime}}\right)\right\|_{L^{1}\left(0, T ; L^{1}(\Omega)\right)} \\
& \quad+\left\|r\left(\theta_{n}\right)-r\left(\theta_{n^{\prime}}\right)\right\|_{L^{1}\left(0, T ; L^{1}(\Omega)\right)}+\left\|\theta_{\delta_{n}}^{b}-\theta_{\delta_{n^{\prime}}}^{b}\right\|_{L^{1}((0, T) \times \omega)}+\left\|c\left(\theta_{\delta_{n}}^{0}-\theta_{\delta_{n^{\prime}}}^{0}\right)\right\|_{L^{1}(\Omega)} .
\end{aligned}
$$

Next, we observe that

$$
\Phi_{0}(s)= \begin{cases}\frac{s^{2}}{2} & \text { if }|s| \leq 1 \\ s-\frac{1}{2} & \text { if } s>1 \\ -s-\frac{1}{2} & \text { if } s<-1\end{cases}
$$

which implies $\Phi_{0}(s) \geq \frac{|s|}{2}$ if $|s| \geq 1$. It follows that

$$
\begin{aligned}
& c_{0} \int_{\Omega}\left|\theta_{n}(t, x)-\theta_{n^{\prime}}(t, x) d x\right| \\
& \quad \leq c_{0}\left\{\left(\int_{\Omega} \Phi_{0}\left(\theta_{n}(t, x)-\theta_{n^{\prime}}(t, x)\right)\right)^{1 / 2} \operatorname{meas}(\Omega)^{1 / 2}+2 \int_{\Omega} \Phi_{0}\left(\theta_{n}(t, x)-\theta_{n^{\prime}}(t, x)\right)\right\},
\end{aligned}
$$

which allows us to conclude that $\left(\theta_{n}\right)_{n \geq 1}$ is a Cauchy sequence in $C^{0}\left([0, T] ; L^{1}(\Omega)\right)$. As a consequence, we may relax the regularity of the test-function $\widetilde{\zeta}$ from $C^{\infty}([0, T])$ in (2.15).

Moreover, following [28], we can prove that $\theta$ is an entropy solution of (1.3)-(1.9). For more details on the definition of entropy (or renormalized) solutions, the reader is referred to $[5,12,28]$ and the references therein.

Finally, let us emphasize that the strong convergence of $\left(\theta_{n}\right)_{n \geq 1}$ to $\theta$ in the space $C^{0}\left([0, T] ; L^{1}(\Omega)\right)$, which is the key point to show that $\theta$ is an entropy solution of (1.3)-(1.9), will not be true anymore if convective effects are taken into account in the heat equation.

\section{Acknowledgements}

No acknowledgements.

\section{Funding}

No funding. 


\section{Declarations}

\section{Competing interests}

The author declares that she has no competing interests.

\section{Authors' contributions}

I declare that I am the sole author of this work. The author read and approved the final manuscript.

\section{Authors' information}

Lyon University, F-42023 Saint-Etienne, Institut Camille Jordan CNRS UMR 5208, 23 Rue Michelon, 42023 Saint-Etienne Cedex 2, France.

\section{Publisher's Note}

Springer Nature remains neutral with regard to jurisdictional claims in published maps and institutional affiliations.

Received: 22 September 2021 Accepted: 12 January 2022 Published online: 24 January 2022

\section{References}

1. Al Zahrani, S.M.: A generalized rheological model for shear thinning fluids. J. Pet. Sci. Eng. 17(3/4), 211-215 (1997)

2. Amrouche, C., Girault, V.: Decomposition of vector spaces and application to the Stokes problem in arbitrary dimension. Czechoslov. Math. J. 44, 109-140 (1994)

3. Barnes, H.A.: Shear-thickening (dilatancy) in suspensions on nonaggregating solid particles dispersed in Newtonian liquids. J. Rheol. 33(2), 329-366 (1989)

4. Barnes, H.A.: A review of the slip (wall depletion) of polymer solutions, emulsions and particle suspensions in viscometers: its cause, character, and cure. J. Non-Newton. Fluid Mech. 56, 221-251 (1995)

5. Blanchard, D., Murat, F.: Renormalized solutions of nonlinear parabolic problems with $L^{1}$ data: existence and uniqueness. Proc. R. Soc. Edinb., Sect. A 127, 1137-1152 (1997)

6. Boccardo, L., Gallouët, T.: Non-linear elliptic and parabolic equations involving measure data. J. Funct. Anal. 87, 149-169 (1989)

7. Boukrouche, M., Boussetouan, I., Paoli, L.: Non-isothermal Navier-Stokes system with mixed boundary conditions and friction law: uniqueness and regularity properties. Nonlinear Anal., Theory Methods Appl. 102, 168-185 (2014)

8. Boukrouche, M., Boussetouan, I., Paoli, L.: Unsteady 3D-Navier-Stokes system with Tresca's friction boundary conditions law. Q. Appl. Math. 78(3), 525-543 (2020)

9. Boukrouche, M., Debbiche, H., Paoli, L.: Non-isothermal non-Newtonian fluid flow problem with heat convection and Tresca's friction law. Preprint (2021). arXiv:2112.07266

10. Boukrouche, M., Debbiche, H., Paoli, L.: Unsteady non-Newtonian fluid flows with boundary conditions of friction type: the case of shear thinning fluids. Preprint (2021). arXiv:2112.07834

11. Boukrouche, M., Debbiche, H., Paoli, L.: Unsteady non-Newtonian fluid flows with boundary conditions of friction type: the case of shear thickening fluids. Nonlinear Anal., Theory Methods Appl. 216, 112701 (2022). https://doi.org/10.1016/j.na.2021.112701

12. Droniou, J., Prignet, A.: Equivalence between entropy and renormalized solutions for parabolic equations with smooth measure data. NoDEA Nonlinear Differ. Equ. Appl. 14, 181-205 (2007)

13. El Kissi, N., Piau, J.M.: Slip and friction of polymer melt flows. Rheol. Ser. 5, 357-388 (1996)

14. Fujita, H.: Flow Problems with Unilateral Boundary Conditions. Collège de France, Leçons (1993)

15. Fujita, H.: A mathematical analysis of motions of viscous incompressible fluid under leak or slip boundary conditions. Math. Fluid Mech. Model. 888, 199-216 (1994)

16. Fujita, H.: Remarks on the Stokes flows under slip and leak boundary conditions of friction type. Top. Math. Fluid Mech. 10, 73-94 (2002)

17. Fujita, H.: A coherent analysis of Stokes flows under boundary conditions of friction type. J. Comput. Appl. Math. 149, 57-69 (2002)

18. Fujita, H., Kawarada, H.: Variational inequalities for the Stokes equation with boundary conditions of friction type. Recent Developments in Domain Decomposition Methods and Flow Problems, GAKUTO Int. Ser. Math. Sci. Appl. 11, 15-33 (1998)

19. Fujita, H., Kawarada, H., Sasamoto, A.: Analytical and numerical approaches to stationary flow problems with leak and slip boundary conditions. Adv. Numer. Math. 14, 17-31 (1994)

20. Girault, V., Raviart, P.A.: Finite Element Approximation of the Navier Stokes Equations. Springer, Berlin (1986)

21. Hervet, H., Léger, L.: Flow with slip at the wall: from simple to complex fluids. C. R., Phys. 4, 241-249 (2003)

22. Ladyzhenskaya, O.A.: New equations for the description of the motions of viscous incompressible fluids, and global solvability for their boundary value problems. Boundary Value Problems of Mathematical Physics Part 5, Tr. Mat. Inst. Steklova 102, 85-104 (1967)

23. Le Roux, C., Tani, A.: Steady flows of incompressible Newtonian fluids with threshold slip boundary conditions. Math. Anal. Fluid Gas Dyn. 1353, 21-34 (2004)

24. Le Roux, C., Tani, A.: Steady solutions of the Navier-Stokes equations with threshold slip boundary conditions. Math. Methods Appl. Sci. 30, 595-624 (2007)

25. Mewis, J., Wagner, N.J.: Colloidal Suspension Rheology. Cambridge University Press, Cambridge (2012)

26. Pahlavan, A.A., Freund, J.B.: Effect of solid properties on slip at a fluid-solid interface. Phys. Rev. E 83, 021602 (2011)

27. Pit, R., Hervet, H., Léger, L.: Friction and slip of a simple liquid at solid surface. Tribol. Lett. 7, 147-152 (1999)

28. Prignet, A.: Existence and uniqueness of "entropy" solutions of parabolic problems with $L^{1}$ data. Nonlinear Anal., Theory Methods Appl. 28(12), 1943-1954 (1997)

29. Roubicek, T.: Nonlinear Partial Differential Equations with Applications. ISNM, vol. 153. Birkhäuser, Basel (2005)

30. Rudolph, N., Osswald, T.A.: Polymer Rheology, Fundamentals and Applications. Hanser Verlag, München (2015) 
31. Saito, N.: On the Stokes equations with the leak and slip boundary conditions of friction type: regularity of solutions. Publ. Res. Inst. Math. Sci., Ser. A 40, 345-383 (2004)

32. Saito, N., Fujita, H.: Regularity of solutions to the Stokes equation under a certain nonlinear boundary condition. In: The Navier-Stokes Equations. Lecture Note Pure Appl. Math., vol. 223, pp. 73-86 (2001)

33. Shaw, M.T.: Introduction to Polymer Rheology. Wiley, Hoboken (2012)

34. Simon, J.: Compact sets in the space $L^{P}(0, T ; B)$. Ann. Mat. Pura Appl. 146, 65-96 (1987)

35. Sochi, T: Slip at fluid-solid interface. Polym. Rev. 51, 309-340 (2011)

36. Takahito, K.: On a strong solution of the non-stationary Navier-Stokes equations under slip or leak boundary conditions of friction type. J. Differ. Equ. 254, 756-778 (2013)

37. Wagner, N.J., Brady, J.F.: Shear thickening in colloidal dispersions. Phys. Today 62(10), 27-32 (2009)

38. Wang, L.: On Korn's inequality. J. Comput. Math. 21, 321-324 (2003)

Submit your manuscript to a SpringerOpen ${ }^{\circ}$ journal and benefit from:

- Convenient online submission

- Rigorous peer review

- Open access: articles freely available online

- High visibility within the field

- Retaining the copyright to your article

Submit your next manuscript at $\gg$ springeropen.com 